\title{
Absolute Magnitude Calibration for Red Giants Based on the Colour-Magnitude Diagrams of Galactic Clusters. III. Calibration with 2MASS
}

\author{
S. Karaali ${ }^{1,2, *}$, S. Bilir ${ }^{1}$ and E. Yaz Gökçe ${ }^{1}$ \\ ${ }^{1}$ Faculty of Sciences, Department of Astronomy and Space Sciences, Istanbul University, 34119 Istanbul, Turkey \\ ${ }^{2}$ Email: karsa@istanbul.edu.tr
}

(Received June 9, 2012; Accepted August 15, 2012; Online Publication January 18, 2013)

\begin{abstract}
We present two absolute magnitude calibrations, $M_{J}$ and $M_{K_{\mathrm{s}}}$, for red giants with the colour-magnitude diagrams of five Galactic clusters with different metallicities, i.e. M92, M13, M71, M67, and NGC 6791. The combination of the absolute magnitudes of the red giant sequences with the corresponding metallicities provides calibration for absolute magnitude estimation for red giants for a given colour. The calibrations for $M_{J}$ and $M_{K_{\mathrm{s}}}$ are defined in the colour intervals $1.3 \leq(V-J)_{0} \leq 2.8$ and $1.75 \leq\left(V-K_{\mathrm{s}}\right)_{0} \leq 3.80 \mathrm{mag}$, respectively, and they cover the metallicity interval $-2.15 \leq[\mathrm{Fe} / \mathrm{H}] \leq+0.37$ dex. The absolute magnitude residuals obtained by the application of the procedure to another set of Galactic clusters lie in the intervals $-0.08<\Delta M_{J} \leq+0.34$ and $-0.10<\Delta M_{K_{\mathrm{s}}} \leq+0.27$ mag for $M_{J}$ and $M_{K_{\mathrm{s}}}$, respectively. The means and standard deviations of the residuals are $\left\langle\Delta M_{J}\right\rangle=0.137$ and $\sigma_{M_{J}}=0.080$, and $\left\langle\Delta M_{K_{\mathrm{s}}}\right\rangle^{\mathrm{s}}=0.109$ and $\sigma_{M_{K_{\mathrm{s}}}}=0.123 \mathrm{mag}$. The derived relations are applicable to stars older than $4 \mathrm{Gyr}$, the age of the youngest calibrating cluster.
\end{abstract}

Keywords: globular clusters: individual (M13, M71, M92), open clusters and associations: individual (M67, NGC 6791), stars: distances, red giants.

\section{INTRODUCTION}

Systematic studies of star clusters help us to understand the Galactic structure and star formation processes as well as stellar evolution. By utilising colour-magnitude diagrams of the stars observed in the optical/near-infrared (NIR) bands, it is possible to determine the underlying properties of the clusters such as age, metallicity and distance. Colour-magnitude diagrams of star clusters can be used as a good distance indicator. The distance to a star can be evaluated by trigonometric or photometric parallaxes. Trigonometric parallaxes are only available for nearby stars where Hipparcos (ESA 1997) is the main supplier for the data. For stars at large distances, the use of photometric parallaxes is unavoidable. In other words, the study of the Galactic structure is strictly tied to the precise determination of absolute magnitudes.

Different methods can be used for absolute magnitude determination, where most of them are devoted to dwarfs. The method used in the Strömgren's $u v b y-\beta$ (Nissen \& Schuster 1991) and in the $U B V$ (Laird, Carney, \& Latham 1988)

\footnotetext{
${ }^{*}$ Retired.
}

photometry depends on the absolute magnitude offset from a standard main sequence. In recent years, the derivation of absolute magnitudes has been carried out by means of colour-absolute magnitude diagrams of some specific clusters whose metal abundances are generally adopted as the mean metal abundance of a Galactic population, such as thin, thick discs and halo. The studies of Phleps et al. (2000) and Chen et al. (2001) can be given as examples. A slightly different approach is that of Siegel et al. (2002), where two relations, one for stars with solar-like abundances and another for metal-poor stars, were derived between $M_{R}$ and the colour index $R-I$, where $M_{R}$ is the absolute magnitude in the $R$ filter of the Johnson system. For a star of given metallicity and colour, absolute magnitude can be estimated by linear interpolation of two ridgelines and by means of linear extrapolation beyond the metal-poor ridgeline.

The most recent procedure used for absolute magnitude determination consists of finding the most likely values of the stellar parameters, given the measured atmospheric ones, and the time spent by a star in each region of the H-R diagram. In practice, researchers select the subset of isochrones 
Table 1. Data for Five Clusters

\begin{tabular}{|c|c|c|c|c|c|c|}
\hline Cluster & $\begin{array}{l}l \\
\left(^{\circ}\right)\end{array}$ & $\begin{array}{l}b \\
\left({ }^{\circ}\right)\end{array}$ & $\begin{array}{c}E(B-V) \\
\quad(\mathrm{mag})\end{array}$ & $\begin{array}{c}\mu_{0} \\
(\mathrm{mag})\end{array}$ & $\begin{array}{c}{[\mathrm{Fe} / \mathrm{H}]} \\
(\operatorname{dex})\end{array}$ & Ref. \\
\hline \multirow[t]{3}{*}{ M92 } & 68.34 & +34.86 & 0.025 & 14.72 & -2.15 & 1 \\
\hline & & & 0.020 & 14.59 & -2.31 & 2 \\
\hline & & & 0.023 & 14.55 & -2.40 & 3 \\
\hline \multirow[t]{3}{*}{ M13 } & 59.01 & +40.91 & 0.020 & 14.38 & -1.41 & 1 \\
\hline & & & 0.020 & 14.27 & -1.53 & 2 \\
\hline & & & 0.016 & 14.35 & -1.60 & 3 \\
\hline \multirow[t]{3}{*}{ M71 } & 56.75 & -4.56 & 0.280 & 12.83 & -0.78 & 4 \\
\hline & & & 0.250 & 13.03 & -0.78 & 2 \\
\hline & & & 0.220 & 13.10 & -0.80 & 3 \\
\hline \multirow[t]{3}{*}{ M67 } & 215.70 & +31.90 & 0.038 & 9.53 & -0.04 & 1 \\
\hline & & & 0.041 & 9.59 & -0.009 & 5 \\
\hline & & & 0.050 & 9.43 & -0.09 & 6 \\
\hline \multirow[t]{3}{*}{ NGC 6791} & 69.66 & +10.90 & 0.150 & 13.10 & 0.37 & 3 \\
\hline & & & 0.150 & 13.14 & 0.45 & 7 \\
\hline & & & 0.100 & 12.94 & 0.37 & 8 \\
\hline
\end{tabular}

Notes. We used the data in the first line for each cluster for absolute magnitude calibration, whereas those in the second and third lines are for comparison purposes. $l$ and $b$ are the Galactic longitude and latitude of the clusters; the symbol $\mu_{0}$ indicates the true distance modulus of the cluster. References: (1) Gratton et al. (1997); (2) Harris (2010); (3) Brasseur et al. (2010); (4) Hodder et al. (1992); (5) Sarajedini, Dotter, \& Kirkpatrick (2009); (6) Hog \& Flynn (1998); (7) Anthony-Twarog, Twarog, \& Mayer (2007); (8) Sandage, Lubin, \& VandenBerg (2003).

with $[\mathrm{M} / \mathrm{H}] \pm \Delta_{[\mathrm{M} / \mathrm{H}]}$, where $\Delta_{[\mathrm{M} / \mathrm{H}]}$ is the estimated error on the metallicity, for each set of derived $T_{\text {eff }}, \log g$ and $[\mathrm{M} / \mathrm{H}]$. Then, a Gaussian weight is associated with each point of the selected isochrones, which depends on the measured atmospheric parameters and the errors considered. This criterion allows the algorithm to select only the points whose values are closed by the pipeline. For details of this procedure, we cite the works of Breddels et al. (2010) and Zwitter et al. (2010). This procedure is based on many parameters. Hence, it provides absolute magnitudes with high accuracy. Also, it can be applied to both dwarf and giant stars simultaneously.

In Karaali et al. (2003), we presented a procedure for the photometric parallax estimation of dwarf stars which depends on the absolute magnitude offset from the main sequence of the Hyades cluster. Bilir et al. (2008) obtained the absolute magnitude calibrations of the thin disc main-sequence stars in the optical $M_{V}$ and in the NIR $M_{J}$ bands using the recent reduced Hipparcos astrometric data (van Leeuwen 2007). Bilir et al. (2009) derived a new luminosity colour relation based on trigonometric parallaxes for the thin disc main-sequence stars with Sloan Digital Sky Survey (SDSS) photometry. Yaz et al. (2010) obtained transformation between optical and NIR bands for red giants. Bilir et al. (2012) extended this study to mid-infrared bands by using Radial Velocity Experiment (RAVE) Third Data Release (DR3) data (Siebert et al. 2011). Both works provide absolute magnitudes for a given photometry from another one.

In Karaali, Bilir, \& Yaz Gökçe (2012a, 2012b; hereafter Paper I and Paper II, respectively), we used a procedure for the absolute magnitude estimation of red giants by using the $V_{0} \times(B-V)_{0}$ and $g_{0} \times(g-r)_{0}$ apparent magnitudecolour diagrams of Galactic clusters with different metallicities. Here, we extend our procedure to Two Micron All Sky
Survey (2MASS; Skrutskie et al. 2006) photometry. We aim to estimate $M_{J}$ and $M_{K}$ absolute magnitudes for red giants with $J_{0} \times(V-J)_{0}$ and $K_{\mathrm{s}_{0}} \times\left(V-K_{\mathrm{s}}\right)_{0}$ colour-magnitude diagrams. The outline of the paper is as follows. We present the data in Section 2. The procedure used for calibration is given in Section 3, and Section 4 is devoted to summary and discussion.

\section{DATA}

We calibrated two different absolute magnitudes, $M_{J}$ and $M_{K_{\mathrm{s}}}$, in terms of metallicity. Hence, we used two different sets of data. The calibration of $M_{J}$ with $J_{0}$ and $(V-J)_{0}$ is given in Section 2.1, whereas that for $M_{K_{\mathrm{s}}}$ with $K_{\mathrm{s}_{0}}$ and $\left(V-K_{\mathrm{s}}\right)_{0}$ is presented in Section 2.2.

\subsection{Data for Calibration with $J_{0}$ and $(V-J)_{0}$}

Five clusters with different metallicities, i.e. M92, M13, M71, M67, and NGC 6791, were selected for our program (Table 1). The $V$ magnitudes and $V-J$ colours for the clusters M92, M13, and M71 were taken from the tables in Brasseur et al. (2010), whereas the data for the clusters NGC 6791 and M67 could be provided by different procedures as explained in the following. We used Figure 1 of Brasseur et al. (2010) and obtained a set of $30\left[M_{V},(V-J)_{0}\right]$ couples for the red giant branch (RGB) of the cluster NGC 6791. Then, we transformed the $M_{V}$ absolute magnitudes to $V$ apparent magnitudes by means of the apparent distance modulus of the cluster, i.e. $\mu=13.25 \mathrm{mag}$. Finally, we de-reddened the $V$ magnitudes and combined them with the true colour indices $(V-J)_{0}$ and obtained $J_{0}$ magnitudes. The $J_{0}$ magnitudes and $(V-J)_{0}$ colours are not available for the cluster M67 


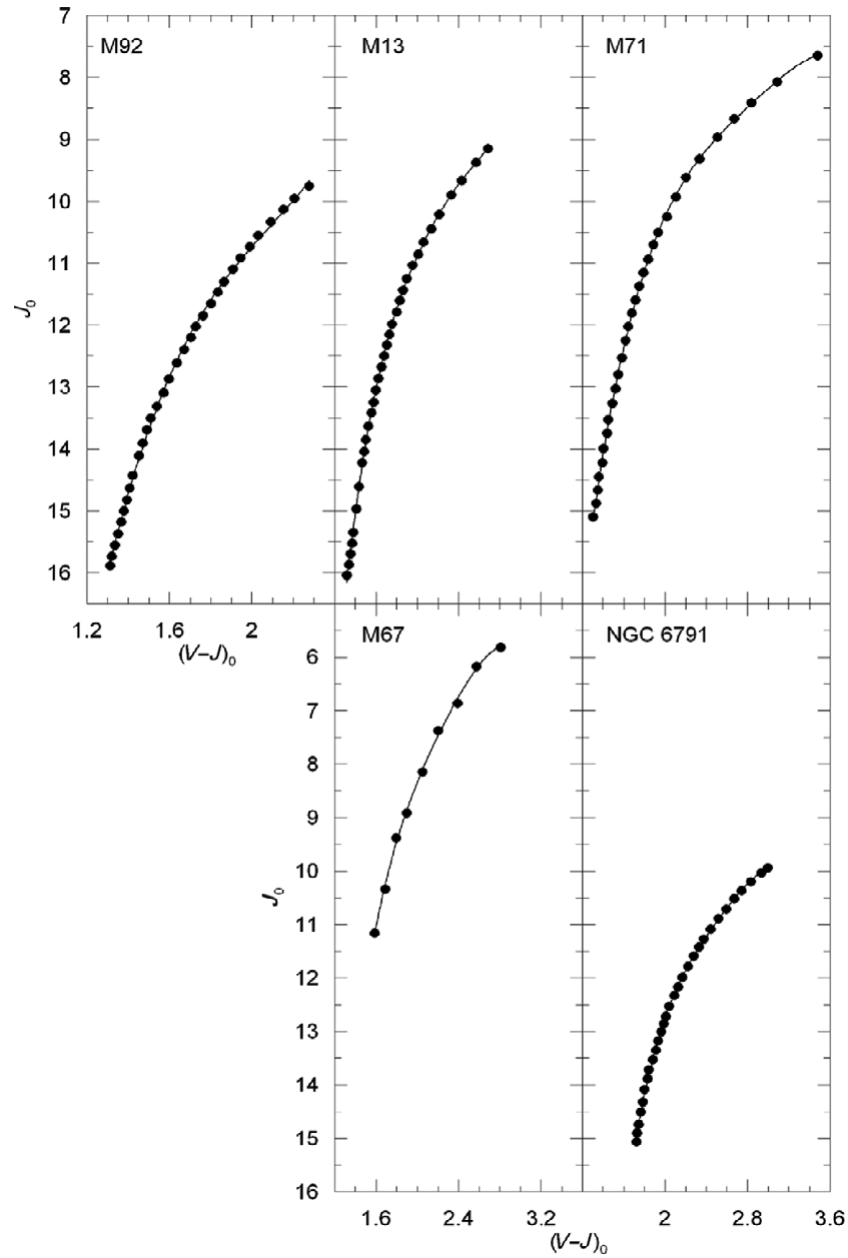

Figure 1. $J_{0} \times(V-J)_{0}$ colour-apparent magnitude diagrams for five Galactic clusters used for the absolute magnitude calibration.

in the literature. Hence, we transformed the $V, B-V$ and $V-I$ data in Montgomery, Marschall, \& Janes (1993) to obtain a set of $\left(J_{0},(V-J)_{0}\right)$ data for the RGB of M67. It turned out that 21 of the stars in the bright stars catalogue in Montgomery et al. (1993) were red giants. We de-reddened the $V, B-V$, and $V-I$ data of these stars and transformed them to $(V-J)_{0}$ colours by using the following equation of Yaz et al. (2010):

$$
\begin{aligned}
(V-J)_{0}= & 1.080(B-V)_{0}+0.379(V-I)_{0} \\
& -0.082[\mathrm{Fe} / \mathrm{H}]+0.279
\end{aligned}
$$

Then, we combined them with the $V_{0}$ magnitudes and obtained the $J_{0}$ ones.

We adopted $R=A_{V} / E(B-V)=3.1$ to convert the colour excess to the extinction. Although different numerical values appeared in the literature for specific regions of our Galaxy, a single value is applicable everywhere. Then, we used the equations $E(V-I) / E(B-V)=1.25$ and $E(V-J) / E(B-V)=2.25$ of Fiorucci \& Munari (2003) and McCall (2004), respectively, to evaluate the colour excesses in $V-I$ and $V-J$ colours. The intrinsic colours were evaluated by the equations $(V-I)_{0}=(V-I)-E(V-I)$ and $(B-V)_{0}=(B-V)-E(B-V)$.

We adopted different equations, i.e. $R=A_{V} / E(B-V)=$ 4.0 (Turner 2012) and $E(V-J) / E(B-V)=2.30$ (Smith 1987), and evaluated the corresponding $E(V-J)$ selective and $A_{J}=1.70 E(B-V)$ total absorptions for the clusters [we do not give the calculations here, only we remind to the reader that $E(V-J)$ is equivalent to $\left.A_{V}-A_{J}\right]$. The results are given in Table 2. The differences between the $E(V-J)$ colour excesses estimated in our study and the ones in this paragraph are rather small. The same case holds for the $A_{J}$ total absorptions except those for M71 and NGC 6791, i.e. $\Delta A_{J} \sim 0.2$ and 0.1 , respectively, whose $E(B-V)$ colour excesses are slightly larger. We should add that the extinction equation of Turner (2012) was derived for low Galactic latitudes, i.e. the Carina region $\left(b \sim 0^{\circ}\right)$. Whereas the Galactic latitudes of the clusters used in our study are (absolutely) greater than $b=4.5$ (see Table 1). Hence, the extinction ratio and the colour excess ratios used in our study are preferable.

The range of the metallicity of the clusters in iron abundance is $-2.15 \leq[\mathrm{Fe} / \mathrm{H}] \leq+0.37 \mathrm{dex}$. The $\mu_{0}$ true distance modulus, $E(B-V)$ colour excess, and $[\mathrm{Fe} / \mathrm{H}]$ iron abundance for M92, M13, M71, and M67 were taken from Paper I, whereas those for the cluster NGC 6791 are taken from Brasseur et al. (2010), except the metallicity which is adopted

Table 2. Comparison of the Selective and Total Absorptions Evaluated by Using Different Extinction and Colour Excess

\begin{tabular}{|c|c|c|c|c|c|c|c|}
\hline $\begin{array}{l}\text { (1) } \\
\text { Cluster }\end{array}$ & $\begin{array}{c}(2) \\
E(B-V)\end{array}$ & $\begin{array}{c}(3) \\
E(V-J)_{p}\end{array}$ & $\begin{array}{c}(4) \\
E(V-J)_{c}\end{array}$ & $\begin{array}{c}(5) \\
\Delta E(V-J)\end{array}$ & $\begin{array}{c}(6) \\
\left(A_{J}\right)_{p}\end{array}$ & $\begin{array}{c}(7) \\
\left(A_{J}\right)_{c}\end{array}$ & $\begin{array}{c}(8) \\
\Delta A_{J}\end{array}$ \\
\hline M92 & 0.025 & 0.056 & 0.058 & 0.002 & 0.022 & 0.043 & 0.021 \\
\hline M13 & 0.020 & 0.045 & 0.046 & 0.001 & 0.017 & 0.034 & 0.017 \\
\hline M71 & 0.280 & 0.630 & 0.644 & 0.014 & 0.244 & 0.476 & 0.232 \\
\hline M67 & 0.038 & 0.086 & 0.087 & 0.001 & 0.033 & 0.065 & 0.032 \\
\hline NGC 6791 & 0.150 & 0.338 & 0.345 & 0.007 & 0.131 & 0.255 & 0.125 \\
\hline
\end{tabular}
Ratios

Notes. Description of columns: (1) the cluster; (2) adopted $E(B-V)$ colour excess; (3) $E(V-J)_{p}$, the colour excess evaluated by the equation $E(V-J) / E(B-V)=2.25$ and used in the paper; (4) $E(V-J)_{c}$, the colour excess evaluated by the equation $E(V-J) / E(B-V)=2.30$ for comparison purposes; (5) $\Delta E(V-J)$, the difference between the colour excesses in columns (3) and (4); (6) $\left(A_{J}\right)_{p}$, the total absorption evaluated by the equation $A_{J} / E(B-V)=0.87$ and used in the paper; (7) $\left(A_{J}\right)_{c}$, the total absorption evaluated by the equation $A_{J} / E(B-V)=1.70$; and (8) $\Delta A_{J}$, the difference between the total absorptions in columns (6) and (7). 
Table 3. $J_{0}$ Magnitudes and $(V-J)_{0}$ Colours for Five Clusters Used for the Absolute Magnitude Calibration

\begin{tabular}{|c|c|c|c|c|c|c|c|c|c|c|c|c|c|c|c|c|c|c|c|}
\hline V & $V-J$ & $J$ & $(V-J)_{0}$ & $J_{0}$ & V & $V-J$ & $J$ & $(V-J)_{0}$ & $J_{0}$ & V & $B-V$ & $V-I$ & $V_{0}$ & $(B-V)_{0}$ & $V-I_{0}$ & $(V-J)_{0}$ & $J_{0}$ & $(V-J)_{0}$ & $J_{0}$ \\
\hline \multicolumn{5}{|c|}{ M92 } & \multicolumn{5}{|c|}{ M13 (cont.) } & \multicolumn{10}{|c|}{ M67 } \\
\hline 12.103 & 2.332 & 9.771 & 2.276 & 9.749 & 14.88 & 1.620 & 13.260 & 1.575 & 13.243 & 8.740 & 1.670 & 2.060 & 8.622 & 1.632 & 2.013 & 2.808 & 5.815 & 2.808 & 5.815 \\
\hline 12.235 & 2.262 & 9.973 & 2.206 & 9.951 & 15.036 & 1.601 & 13.435 & 1.556 & 13.418 & 8.860 & 1.590 & 1.670 & 8.742 & 1.552 & 1.623 & 2.573 & 6.169 & 2.573 & 6.169 \\
\hline 12.362 & 2.208 & 10.154 & 2.152 & 10.132 & 15.214 & 1.569 & 13.645 & 1.524 & 13.628 & 9.370 & 1.480 & 1.500 & 9.252 & 1.442 & 1.453 & 2.390 & 6.862 & 2.390 & 6.862 \\
\hline 12.503 & 2.147 & 10.356 & 2.091 & 10.334 & 15.408 & 1.546 & 13.862 & 1.501 & 13.845 & 9.530 & 1.380 & 1.330 & 9.412 & 1.342 & 1.283 & 2.218 & 7.194 & 2.204 & 7.373 \\
\hline 12.655 & 2.086 & 10.569 & 2.030 & 10.547 & 15.585 & 1.527 & 14.058 & 1.482 & 14.041 & 9.690 & 1.360 & 1.330 & 9.572 & 1.322 & 1.283 & 2.196 & 7.376 & 2.049 & 8.147 \\
\hline 12.798 & 2.046 & 10.752 & 1.990 & 10.730 & 15.747 & 1.511 & 14.236 & 1.466 & 14.219 & 9.720 & 1.370 & 1.360 & 9.602 & 1.332 & 1.313 & 2.218 & 7.384 & 1.897 & 8.915 \\
\hline 12.933 & 1.999 & 10.934 & 1.943 & 10.912 & 16.104 & 1.478 & 14.626 & 1.433 & 14.609 & 9.840 & 1.360 & 1.300 & 9.722 & 1.322 & 1.253 & 2.185 & 7.537 & 1.794 & 9.382 \\
\hline 13.085 & 1.964 & 11.121 & 1.908 & 11.099 & 16.433 & 1.451 & 14.982 & 1.406 & 14.965 & 10.120 & 1.300 & 1.270 & 10.002 & 1.262 & 1.223 & 2.109 & 7.894 & 1.690 & 10.337 \\
\hline 13.236 & 1.920 & 11.316 & 1.864 & 11.294 & 16.786 & 1.424 & 15.362 & 1.379 & 15.345 & 10.300 & 1.260 & 1.230 & 10.182 & 1.222 & 1.183 & 2.050 & 8.132 & 1.585 & 11.158 \\
\hline 13.379 & 1.889 & 11.490 & 1.833 & 11.468 & 16.952 & 1.411 & 15.541 & 1.366 & 15.524 & 10.520 & 1.230 & 1.150 & 10.402 & 1.192 & 1.103 & 1.987 & 8.415 & - & - \\
\hline 13.528 & 1.857 & 11.671 & 1.801 & 11.649 & 17.112 & 1.397 & 15.715 & 1.352 & 15.698 & 10.930 & 1.150 & 1.140 & 10.812 & 1.112 & 1.093 & 1.897 & 8.915 & - & - \\
\hline 13.682 & 1.818 & 11.864 & 1.762 & 11.842 & 17.271 & 1.381 & 15.890 & 1.336 & 15.873 & 11.200 & 1.080 & 1.080 & 11.082 & 1.042 & 1.033 & 1.799 & 9.283 & - & - \\
\hline 13.827 & 1.783 & 12.044 & 1.727 & 12.022 & 17.420 & 1.360 & 16.060 & 1.315 & 16.043 & 11.240 & 1.100 & 1.070 & 11.122 & 1.062 & 1.023 & 1.817 & 9.305 & - & - \\
\hline 13.984 & 1.761 & 12.223 & 1.705 & 12.201 & \multicolumn{5}{|c|}{ M71 } & 11.440 & 1.060 & 1.050 & 11.322 & 1.022 & 1.003 & 1.766 & 9.556 & - & - \\
\hline 14.140 & 1.727 & 12.413 & 1.671 & 12.391 & 11.997 & 4.109 & 7.888 & 3.479 & 7.644 & 12.094 & 1.007 & 1.016 & 11.976 & 0.969 & 0.969 & 1.696 & 10.280 & - & - \\
\hline 14.321 & 1.692 & 12.629 & 1.636 & 12.607 & 12.035 & 3.717 & 8.318 & 3.087 & 8.074 & 12.110 & 1.006 & 1.029 & 11.992 & 0.968 & 0.982 & 1.700 & 10.292 & - & - \\
\hline 14.541 & 1.653 & 12.888 & 1.597 & 12.866 & 12.116 & 3.469 & 8.647 & 2.839 & 8.403 & 12.230 & 0.993 & 1.002 & 12.112 & 0.955 & 0.955 & 1.675 & 10.437 & - & - \\
\hline 14.744 & 1.627 & 13.117 & 1.571 & 13.095 & 12.212 & 3.300 & 8.912 & 2.670 & 8.668 & 12.712 & 0.913 & 0.966 & 12.594 & 0.875 & 0.919 & 1.575 & 11.019 & - & - \\
\hline 14.925 & 1.595 & 13.330 & 1.539 & 13.308 & 12.347 & 3.137 & 9.210 & 2.507 & 8.966 & 12.862 & 0.941 & 0.981 & 12.744 & 0.903 & 0.934 & 1.611 & 11.133 & - & - \\
\hline 15.089 & 1.565 & 13.524 & 1.509 & 13.502 & 12.523 & 2.962 & 9.561 & 2.332 & 9.317 & 12.934 & 0.917 & 0.972 & 12.816 & 0.879 & 0.925 & 1.582 & 11.234 & - & - \\
\hline 15.256 & 1.547 & 13.709 & 1.491 & 13.687 & 12.693 & 2.833 & 9.860 & 2.203 & 9.616 & 12.934 & 0.919 & 0.940 & 12.816 & 0.881 & 0.893 & 1.572 & 11.244 & - & - \\
\hline 15.456 & 1.525 & 13.931 & 1.469 & 13.909 & 12.906 & 2.733 & 10.173 & 2.103 & 9.929 & $(V-J)_{0}$ & $M_{V}$ & $V$ & $V_{0}$ & $(V-J)_{0}$ & $J_{0}$ & - & - & - & - \\
\hline 15.637 & 1.507 & 14.130 & 1.451 & 14.108 & 13.139 & 2.648 & 10.491 & 2.018 & 10.247 & \multicolumn{6}{|c|}{ NGC 6791} & - & - & - & - \\
\hline 15.929 & 1.479 & 14.450 & 1.423 & 14.428 & 13.305 & 2.562 & 10.743 & 1.932 & 10.499 & 2.994 & -0.175 & 13.395 & 12.930 & 2.994 & 9.936 & - & - & - & - \\
\hline 16.118 & 1.463 & 14.655 & 1.407 & 14.633 & 13.459 & 2.516 & 10.943 & 1.886 & 10.699 & 2.935 & -0.140 & 13.430 & 12.965 & 2.935 & 10.030 & - & - & - & - \\
\hline 16.292 & 1.448 & 14.844 & 1.392 & 14.822 & 13.646 & 2.465 & 11.181 & 1.835 & 10.937 & 2.832 & -0.078 & 13.492 & 13.027 & 2.832 & 10.195 & - & - & - & - \\
\hline 16.453 & 1.435 & 15.018 & 1.379 & 14.996 & 13.818 & 2.422 & 11.396 & 1.792 & 11.152 & 2.742 & 0.007 & 13.577 & 13.112 & 2.742 & 10.370 & - & - & - & - \\
\hline 16.623 & 1.421 & 15.202 & 1.365 & 15.180 & 13.993 & 2.381 & 11.612 & 1.751 & 11.368 & 2.671 & 0.085 & 13.655 & 13.190 & 2.671 & 10.519 & - & - & - & - \\
\hline 16.801 & 1.406 & 15.395 & 1.350 & 15.373 & 14.177 & 2.342 & 11.835 & 1.712 & 11.591 & 2.592 & 0.197 & 13.767 & 13.302 & 2.592 & 10.710 & - & - & - & - \\
\hline 16.970 & 1.391 & 15.579 & 1.335 & 15.557 & 14.356 & 2.306 & 12.050 & 1.676 & 11.806 & 2.514 & 0.295 & 13.865 & 13.400 & 2.514 & 10.886 & - & - & - & - \\
\hline 17.130 & 1.376 & 15.754 & 1.320 & 15.732 & 14.538 & 2.274 & 12.264 & 1.644 & 12.020 & 2.443 & 0.422 & 13.992 & 13.527 & 2.443 & 11.084 & - & - & - & - \\
\hline 17.279 & 1.368 & 15.911 & 1.312 & 15.889 & 14.736 & 2.247 & 12.489 & 1.617 & 12.245 & 2.376 & 0.541 & 14.111 & 13.646 & 2.376 & 11.270 & - & - & - & - \\
\hline & & M13 & & & 14.986 & 2.209 & 12.777 & 1.579 & 12.533 & 2.328 & 0.647 & 14.217 & 13.752 & 2.328 & 11.424 & - & - & - & - \\
\hline 11.895 & 2.729 & 9.166 & 2.684 & 9.1486 & 15.217 & 2.177 & 13.040 & 1.547 & 12.796 & 2.280 & 0.766 & 14.336 & 13.871 & 2.280 & 11.591 & - & - & - & - \\
\hline 12.005 & 2.612 & 9.393 & 2.567 & 9.3756 & 15.426 & 2.152 & 13.274 & 1.522 & 13.030 & 2.221 & 0.899 & 14.469 & 14.004 & 2.221 & 11.783 & - & - & - & - \\
\hline 12.152 & 2.474 & 9.678 & 2.429 & 9.6606 & 15.627 & 2.120 & 13.507 & 1.490 & 13.263 & 2.167 & 1.054 & 14.624 & 14.159 & 2.167 & 11.992 & - & - & - & - \\
\hline 12.289 & 2.373 & 9.916 & 2.328 & 9.8986 & 15.854 & 2.080 & 13.774 & 1.450 & 13.530 & 2.124 & 1.180 & 14.750 & 14.285 & 2.124 & 12.161 & - & - & - & - \\
\hline 12.487 & 2.258 & 10.229 & 2.213 & 10.212 & 16.060 & 2.070 & 13.990 & 1.440 & 13.746 & 2.088 & 1.307 & 14.877 & 14.412 & 2.088 & 12.324 & - & - & - & - \\
\hline 12.642 & 2.182 & 10.460 & 2.137 & 10.443 & 16.268 & 2.035 & 14.233 & 1.405 & 13.989 & 2.040 & 1.461 & 15.031 & 14.566 & 2.040 & 12.526 & - & - & - & - \\
\hline 12.787 & 2.104 & 10.683 & 2.059 & 10.666 & 16.485 & 2.021 & 14.464 & 1.391 & 14.220 & 2.010 & 1.616 & 15.186 & 14.721 & 2.010 & 12.711 & - & - & - & - \\
\hline 12.923 & 2.053 & 10.870 & 2.008 & 10.853 & 16.688 & 1.989 & 14.699 & 1.359 & 14.455 & 1.986 & 1.735 & 15.305 & 14.840 & 1.986 & 12.854 & - & - & - & - \\
\hline 13.051 & 1.999 & 11.052 & 1.954 & 11.035 & 16.885 & 1.977 & 14.908 & 1.347 & 14.664 & 1.963 & 1.862 & 15.432 & 14.967 & 1.963 & 13.004 & - & - & - & - \\
\hline 13.211 & 1.944 & 11.267 & 1.899 & 11.25 & 17.084 & 1.959 & 15.125 & 1.329 & 14.881 & 1.933 & 2.002 & 15.572 & 15.107 & 1.933 & 13.174 & - & - & - & - \\
\hline 13.36 & 1.907 & 11.453 & 1.862 & 11.436 & 17.267 & 1.931 & 15.336 & 1.301 & 15.092 & 1.909 & 2.150 & 15.720 & 15.255 & 1.909 & 13.346 & - & - & - & - \\
\hline 13.499 & 1.875 & 11.624 & 1.830 & 11.607 & - & - & - & - & - & 1.878 & 2.297 & 15.867 & 15.402 & 1.878 & 13.524 & - & - & - & - \\
\hline 13.646 & 1.843 & 11.803 & 1.798 & 11.786 & - & - & - & - & - & 1.842 & 2.445 & 16.015 & 15.550 & 1.842 & 13.708 & - & - & - & - \\
\hline 13.800 & 1.802 & 11.998 & 1.757 & 11.981 & - & - & - & - & - & 1.830 & 2.606 & 16.176 & 15.711 & 1.830 & 13.881 & - & - & - & - \\
\hline 13.945 & 1.774 & 12.171 & 1.729 & 12.154 & - & - & - & - & - & 1.800 & 2.782 & 16.352 & 15.887 & 1.800 & 14.087 & - & - & - & - \\
\hline 14.088 & 1.748 & 12.340 & 1.703 & 12.323 & - & - & - & - & - & 1.782 & 2.993 & 16.563 & 16.098 & 1.782 & 14.316 & - & - & - & - \\
\hline 14.238 & 1.722 & 12.516 & 1.677 & 12.499 & - & - & - & - & - & 1.764 & 3.161 & 16.731 & 16.266 & 1.764 & 14.502 & - & - & - & - \\
\hline 14.393 & 1.697 & 12.696 & 1.652 & 12.679 & - & - & - & - & - & 1.746 & 3.380 & 16.950 & 16.485 & 1.746 & 14.739 & - & - & - & - \\
\hline 14.546 & 1.667 & 12.879 & 1.622 & 12.862 & - & - & - & - & - & 1.728 & 3.520 & 17.090 & 16.625 & 1.728 & 14.897 & - & - & - & - \\
\hline 14.715 & 1.643 & 13.072 & 1.598 & 13.055 & - & - & - & - & - & 1.722 & 3.682 & 17.252 & 16.787 & 1.722 & 15.065 & - & - & - & - \\
\hline
\end{tabular}

Note. The last two columns for the cluster M67 indicate the $(V-I)_{0}$ colours and $J_{0}$ magnitudes of the bins used to draw the diagram of M67 in Figure 1 . 


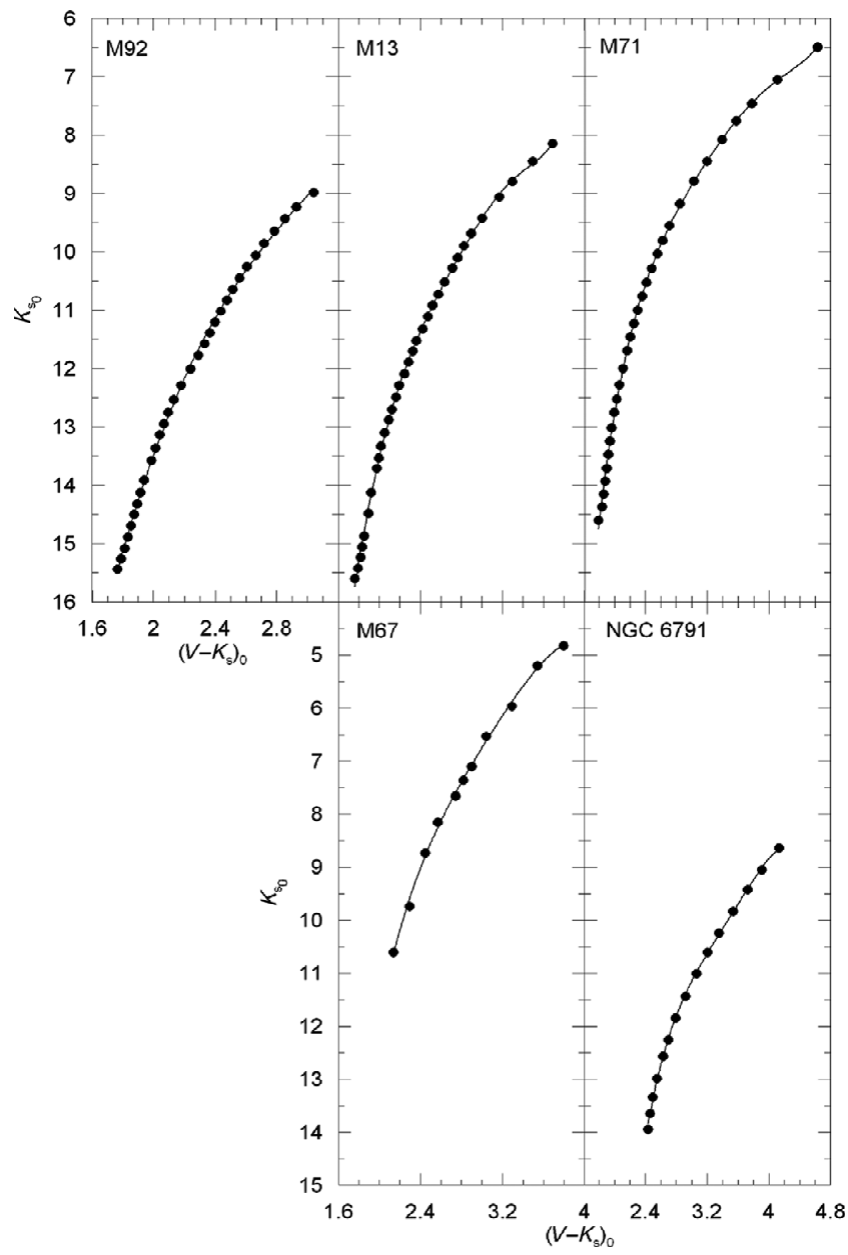

Figure 2. $K_{\mathrm{s}_{0}} \times\left(V-K_{\mathrm{s}}\right)_{0}$ colour-apparent magnitude diagrams for five Galactic clusters used for the absolute magnitude calibration.

from Paper I. These data are given in Table 1 , whereas the $J_{0}$ magnitudes and $(V-J)_{0}$ colours are presented in Table 3. We then fitted the fiducial sequence of the red giants to a fourth-degree polynomial for all clusters. The calibration of $J_{0}$ is as follows:

$$
J_{0}=\sum_{i=0}^{4} a_{i}(V-J)_{0}^{i} .
$$

The numerical values of the coefficients $a_{i}(i=1,2,3,4)$ are given in Table 4 and the corresponding diagrams are pre- sented in Figure 1. The $(V-J)_{0}$ interval in the second line of the table denotes the range of $(V-J)_{0}$ available for each cluster.

\subsection{Data for Calibration with $K_{\mathrm{s}_{0}}$ and $\left(V-K_{\mathrm{s}}\right)_{0}$}

We used the data of the same clusters as mentioned in Section 2.1, i.e. M92, M13, M71, M67, and NGC 6791, for calibration of the $M_{K_{\mathrm{s}}}$ absolute magnitudes. We adopted the same colour excesses, distance moduli, and metallicities as in Table 1. The $K_{\mathrm{s}_{0}}$ magnitudes and $\left(V-K_{\mathrm{s}}\right)_{0}$ colours for the clusters M92, M13, and M71 were taken from the tables of Brasseur et al. (2010). However, we used two different procedures for evaluation of the $K_{\mathrm{s}}$ and $V-K_{\mathrm{s}}$ data for the clusters M67 and NGC 6791, as explained in the following. For the cluster M67, we transformed the $V, B-V$, and $V-I$ data of Montgomery et al. (1993) to $\left(V-K_{\mathrm{s}}\right)_{0}$ by the following equation of Yaz et al. (2010), and then we evaluated the $K_{\mathrm{s}_{0}}$ magnitudes by the combination of $V_{0}$ magnitudes and $\left(V-K_{\mathrm{s}}\right)_{0}$ colours:

$$
\begin{aligned}
\left(V-K_{\mathrm{s}}\right)_{0}= & 1.791(B-V)_{0}+0.294(V-I)_{0} \\
& -0.129[\mathrm{Fe} / \mathrm{H}]+0.279
\end{aligned}
$$

The available 2MASS photometric data in Brasseur et al. (2010) for the cluster NGC 6791 are the $J-K_{\mathrm{s}}$ colours and $K_{\mathrm{s}}$ magnitudes, but not the $V-K_{\mathrm{s}}$ ones. Hence, we evaluated them by means of the $V_{0}$ and $J_{0}$ magnitudes for this cluster given in Table 3 in three steps. First, we plotted $V_{0}$ magnitudes versus $J_{0}$ magnitudes in a diagram (not given here) and obtained the following quadratic equation with a high correlation coefficient, $R^{2}=0.9997$ :

$$
V_{0}=0.0457 J_{0}^{2}-0.3744 J_{0}+12.9000 .
$$

In the second step, we evaluated the $J$ magnitudes by combining $J-K_{\mathrm{s}}$ and $K_{\mathrm{s}}$, and finally we de-reddened the $J$ magnitudes and transformed them to $V_{0}$ magnitudes by Equation (4). We used the equations $A_{J} / E(B-V)=0.87$ and $A_{K_{\mathrm{s}}} / E(B-V)=0.38$ of Savage \& Mathis (1979) for dereddening of the magnitudes $J$ and $K_{\mathrm{s}}$, respectively. As Equation (4) is defined for $9.94 \leq J_{0} \leq 15.06$, we could not consider five bright $J_{0}$ magnitudes in Table 3 . The $\left(V-K_{\mathrm{s}}\right)_{0}$ and $K_{\mathrm{s}_{0}}$ data for the clusters are given in Table 5.

\begin{tabular}{|c|c|c|c|c|c|}
\hline $\begin{array}{l}\text { Cluster } \rightarrow \\
(V-J)_{0}\end{array}$ & $\begin{array}{c}\text { M92 } \\
{[1.30,2.28]}\end{array}$ & $\begin{array}{c}\text { M13 } \\
{[1.30,2.68]}\end{array}$ & $\begin{array}{c}\text { M71 } \\
{[1.30,3.48]}\end{array}$ & $\begin{array}{c}\text { M67 } \\
{[1.58,2.81]}\end{array}$ & $\begin{array}{c}\text { NGC } 6791 \\
{[1.72,2.99]}\end{array}$ \\
\hline$a_{4}$ & 8.7460 & 2.1654 & 0.5646 & 3.0424 & 3.4182 \\
\hline$a_{3}$ & -67.6790 & -20.4910 & -6.5667 & -27.8780 & -34.682 \\
\hline$a_{2}$ & 198.3500 & 73.7370 & 28.9320 & 97.1910 & 132.690 \\
\hline$a_{1}^{2}$ & -265.4200 & -121.9800 & -58.6600 & -156.3900 & -229.450 \\
\hline$a_{0}$ & 149.6300 & 89.1650 & 55.3480 & 106.7000 & 163.660 \\
\hline
\end{tabular}

As in Section 2.1, we adopted different equations, i.e. $R=$ $A_{V} / E(B-V)=4.0($ Turner 2012), $E(J-H) / E(B-V)=$

Table 4. Numerical Values of the Coefficients $a_{i}(i=1,2,3,4)$ in Equation (2) 
Table 5. $K_{\mathrm{s}_{0}}$ Magnitudes and $\left(V-K_{\mathrm{s}}\right)_{0}$ Colours for Five Clusters Used for the Absolute Magnitude Calibration

\begin{tabular}{|c|c|c|c|c|c|c|c|c|c|c|c|c|c|c|}
\hline$V$ & $V-K_{\mathrm{s}}$ & $K_{\mathrm{s}}$ & $\left(V-K_{\mathrm{s}}\right)_{0}$ & $K_{\mathrm{s}_{0}}$ & V & $V-K_{\mathrm{s}}$ & $K_{\mathrm{s}}$ & $\left(V-K_{\mathrm{s}}\right)_{0}$ & $K_{\mathrm{s}_{0}}$ & & & & & \\
\hline & & M92 & & & & & M71 (col & & & & & & & \\
\hline 12.103 & 3.108 & 8.995 & 3.039 & 8.9855 & 12.347 & 4.164 & 8.183 & 3.391 & 8.077 & - & - & - & - & - \\
\hline 12.235 & 2.998 & 9.237 & 2.929 & 9.2275 & 12.523 & 3.970 & 8.553 & 3.197 & 8.447 & - & - & - & - & - \\
\hline 12.362 & 2.922 & 9.440 & 2.853 & 9.4305 & 12.693 & 3.800 & 8.893 & 3.027 & 8.787 & - & - & - & - & - \\
\hline 12.503 & 2.854 & 9.649 & 2.785 & 9.6395 & 12.906 & 3.619 & 9.287 & 2.846 & 9.181 & - & - & - & - & - \\
\hline 12.655 & 2.787 & 9.868 & 2.718 & 9.8585 & 13.139 & 3.481 & 9.658 & 2.708 & 9.552 & - & - & - & - & - \\
\hline 12.798 & 2.730 & 10.068 & 2.661 & 10.059 & 13.305 & 3.390 & 9.915 & 2.617 & 9.809 & - & - & - & - & - \\
\hline 12.933 & 2.675 & 10.258 & 2.606 & 10.249 & 13.459 & 3.324 & 10.135 & 2.551 & 10.029 & - & - & - & - & - \\
\hline 13.085 & 2.626 & 10.459 & 2.557 & 10.450 & 13.646 & 3.250 & 10.396 & 2.477 & 10.290 & - & - & - & - & - \\
\hline 13.236 & 2.583 & 10.653 & 2.514 & 10.644 & 13.818 & 3.187 & 10.631 & 2.414 & 10.525 & - & - & - & - & - \\
\hline 13.379 & 2.544 & 10.835 & 2.475 & 10.826 & 13.993 & 3.129 & 10.864 & 2.356 & 10.758 & - & - & - & - & - \\
\hline 13.528 & 2.505 & 11.023 & 2.436 & 11.014 & 14.177 & 3.071 & 11.106 & 2.298 & 11.000 & - & - & - & - & - \\
\hline 13.682 & 2.467 & 11.215 & 2.398 & 11.206 & 14.356 & 3.020 & 11.336 & 2.247 & 11.230 & - & - & - & - & - \\
\hline 13.827 & 2.433 & 11.394 & 2.364 & 11.385 & 14.538 & 2.974 & 11.564 & 2.201 & 11.458 & - & - & - & - & - \\
\hline 13.984 & 2.399 & 11.585 & 2.330 & 11.576 & 14.736 & 2.935 & 11.801 & 2.162 & 11.695 & - & - & - & - & - \\
\hline 14.140 & 2.357 & 11.783 & 2.288 & 11.774 & 14.986 & 2.880 & 12.106 & 2.107 & 12.000 & - & - & - & - & - \\
\hline 14.321 & 2.308 & 12.013 & 2.239 & 12.004 & 15.217 & 2.835 & 12.382 & 2.062 & 12.276 & - & - & - & - & - \\
\hline 14.541 & 2.247 & 12.294 & 2.178 & 12.285 & 15.426 & 2.798 & 12.628 & 2.025 & 12.522 & - & - & - & - & - \\
\hline 14.744 & 2.201 & 12.543 & 2.132 & 12.534 & 15.627 & 2.766 & 12.861 & 1.993 & 12.755 & - & - & - & - & - \\
\hline 14.925 & 2.162 & 12.763 & 2.093 & 12.754 & 15.854 & 2.734 & 13.120 & 1.961 & 13.014 & - & - & - & - & - \\
\hline 15.089 & 2.136 & 12.953 & 2.067 & 12.944 & 16.060 & 2.709 & 13.351 & 1.936 & 13.245 & - & - & - & - & - \\
\hline 15.256 & 2.109 & 13.147 & 2.040 & 13.138 & 16.268 & 2.687 & 13.581 & 1.914 & 13.475 & - & - & - & - & - \\
\hline 15.456 & 2.080 & 13.376 & 2.011 & 13.367 & 16.485 & 2.667 & 13.818 & 1.894 & 13.712 & - & - & - & - & - \\
\hline 15.637 & 2.054 & 13.583 & 1.985 & 13.574 & 16.688 & 2.649 & 14.039 & 1.876 & 13.933 & - & - & - & - & - \\
\hline 15.929 & 2.005 & 13.924 & 1.936 & 13.915 & 16.885 & 2.631 & 14.254 & 1.858 & 14.148 & - & - & - & - & - \\
\hline 16.118 & 1.982 & 14.136 & 1.913 & 14.127 & 17.084 & 2.606 & 14.478 & 1.833 & 14.372 & - & - & - & - & - \\
\hline 16.292 & 1.961 & 14.331 & 1.892 & 14.322 & 17.267 & 2.564 & 14.703 & 1.791 & 14.597 & - & - & - & - & - \\
\hline 16.453 & 1.942 & 14.511 & 1.873 & 14.502 & $J-K_{\mathrm{s}}$ & $K_{\mathrm{s}}$ & $J$ & $J_{0}$ & $V_{0}$ & $K_{\mathrm{s}_{0}}$ & $\left(V-K_{\mathrm{s}}\right)_{0}$ & & & \\
\hline 16.623 & 1.922 & 14.701 & 1.853 & 14.692 & & & & NGC 6791 & & & & & - & - \\
\hline 16.801 & 1.901 & 14.900 & 1.832 & 14.891 & 1.111 & 8.693 & 9.804 & 9.717 & 12.767 & 8.636 & 4.131 & - & - & - \\
\hline 16.970 & 1.880 & 15.090 & 1.811 & 15.081 & 1.060 & 9.103 & 10.163 & 10.076 & 12.957 & 9.046 & 3.911 & - & - & - \\
\hline 17.130 & 1.857 & 15.273 & 1.788 & 15.264 & 1.016 & 9.478 & 10.494 & 10.407 & 13.143 & 9.421 & 3.722 & - & - & - \\
\hline \multirow[t]{2}{*}{17.279} & 1.832 & 15.447 & 1.763 & 15.438 & 0.978 & 9.887 & 10.865 & 10.778 & 13.363 & 9.830 & 3.533 & - & - & - \\
\hline & & M13 & & & 0.930 & 10.297 & 11.227 & 11.140 & 13.591 & 10.240 & 3.351 & - & - & - \\
\hline 11.895 & 3.741 & 8.154 & 3.686 & 8.146 & 0.901 & 10.661 & 11.562 & 11.475 & 13.811 & 10.604 & 3.207 & - & - & - \\
\hline
\end{tabular}


Table 5. Continued.

\begin{tabular}{|c|c|c|c|c|c|c|c|c|c|c|c|c|c|c|}
\hline$V$ & $V-K_{\mathrm{s}}$ & $K_{\mathrm{s}}$ & $\left(V-K_{\mathrm{s}}\right)_{0}$ & $K_{\mathrm{s}_{0}}$ & V & $V-K_{\mathrm{s}}$ & $K_{\mathrm{s}}$ & $\left(V-K_{\mathrm{s}}\right)_{0}$ & $K_{\mathrm{s}_{0}}$ & & & & & \\
\hline \multicolumn{5}{|c|}{ M92 } & \multicolumn{5}{|c|}{ M71 (cont.) } & & & & & \\
\hline 12.005 & 3.547 & 8.458 & 3.492 & 8.450 & 0.870 & 11.060 & 11.930 & 11.843 & 14.066 & 11.003 & 3.063 & - & - & - \\
\hline 12.152 & 3.352 & 8.800 & 3.297 & 8.792 & 0.835 & 11.493 & 12.328 & 12.241 & 14.355 & 11.436 & 2.919 & - & - & - \\
\hline 12.289 & 3.223 & 9.066 & 3.168 & 9.058 & 0.800 & 11.903 & 12.703 & 12.616 & 14.640 & 11.846 & 2.794 & - & - & - \\
\hline 12.487 & 3.058 & 9.429 & 3.003 & 9.421 & 0.784 & 12.312 & 13.096 & 13.009 & 14.953 & 12.255 & 2.698 & - & - & - \\
\hline 12.642 & 2.952 & 9.690 & 2.897 & 9.682 & 0.762 & 12.617 & 13.379 & 13.292 & 15.188 & 12.560 & 2.628 & - & - & - \\
\hline 12.787 & 2.880 & 9.907 & 2.825 & 9.899 & 0.739 & 13.039 & 13.778 & 13.691 & 15.530 & 12.982 & 2.548 & - & - & - \\
\hline 12.923 & 2.818 & 10.105 & 2.763 & 10.097 & 0.721 & 13.390 & 14.111 & 14.024 & 15.827 & 13.333 & 2.494 & - & - & - \\
\hline 13.051 & 2.768 & 10.283 & 2.713 & 10.275 & 0.708 & 13.705 & 14.413 & 14.326 & 16.106 & 13.648 & 2.458 & - & - & - \\
\hline 13.211 & 2.689 & 10.522 & 2.634 & 10.514 & 0.701 & 13.999 & 14.700 & 14.613 & 16.378 & 13.942 & 2.436 & - & - & - \\
\hline 13.360 & 2.628 & 10.732 & 2.573 & 10.724 & 1.168 & 8.225 & 9.393 & 9.306 & - & - & - & - & - & - \\
\hline 13.499 & 2.573 & 10.926 & 2.518 & 10.918 & 1.229 & 7.768 & 8.997 & 8.910 & - & - & - & - & - & - \\
\hline 13.646 & 2.529 & 11.117 & 2.474 & 11.109 & 1.276 & 7.453 & 8.729 & 8.642 & - & - & - & - & - & - \\
\hline 13.800 & 2.475 & 11.325 & 2.420 & 11.317 & 1.330 & 7.124 & 8.454 & 8.367 & - & - & - & - & - & - \\
\hline 13.945 & 2.415 & 11.530 & 2.360 & 11.522 & 1.362 & 6.901 & 8.263 & 8.176 & - & - & - & - & - & - \\
\hline 14.088 & 2.379 & 11.709 & 2.324 & 11.701 & V & $B-V$ & $V-I$ & $V_{0}$ & $(B-V)_{0}$ & $(V-I)_{0}$ & $\left(V-K_{\mathrm{s}}\right)_{0}$ & $K_{\mathrm{s}}$ & $\left(V-K_{\mathrm{s}}\right)_{0}$ & $K_{\mathrm{s}_{\mathrm{s}}}$ \\
\hline 14.238 & 2.342 & 11.896 & 2.287 & 11.888 & \multicolumn{10}{|c|}{ M67 } \\
\hline 14.393 & 2.298 & 12.095 & 2.243 & 12.087 & 8.740 & 1.670 & 2.060 & 8.622 & 1.632 & 2.013 & 3.796 & 4.826 & 2.135 & 10.608 \\
\hline 14.546 & 2.249 & 12.297 & 2.194 & 12.289 & 8.860 & 1.590 & 1.670 & 8.742 & 1.552 & 1.623 & 3.538 & 5.204 & 2.292 & 9.735 \\
\hline 14.715 & 2.215 & 12.500 & 2.160 & 12.492 & 9.370 & 1.480 & 1.500 & 9.252 & 1.442 & 1.453 & 3.291 & 5.961 & 2.447 & 8.729 \\
\hline 14.880 & 2.173 & 12.707 & 2.118 & 12.699 & 9.530 & 1.380 & 1.330 & 9.412 & 1.342 & 1.283 & 3.062 & 6.350 & 2.570 & 8.157 \\
\hline 15.036 & 2.145 & 12.891 & 2.090 & 12.883 & 9.690 & 1.360 & 1.330 & 9.572 & 1.322 & 1.283 & 3.026 & 6.546 & 2.740 & 7.662 \\
\hline 15.214 & 2.104 & 13.110 & 2.049 & 13.102 & 9.720 & 1.370 & 1.360 & 9.602 & 1.332 & 1.313 & 3.053 & 6.550 & 2.817 & 7.365 \\
\hline 15.408 & 2.072 & 13.336 & 2.017 & 13.328 & 9.840 & 1.360 & 1.300 & 9.722 & 1.322 & 1.253 & 3.017 & 6.705 & 2.901 & 7.101 \\
\hline 15.585 & 2.050 & 13.535 & 1.995 & 13.527 & 10.120 & 1.300 & 1.270 & 10.002 & 1.262 & 1.223 & 2.901 & 7.101 & 3.039 & 6.538 \\
\hline 15.747 & 2.031 & 13.716 & 1.976 & 13.708 & 10.300 & 1.260 & 1.230 & 10.182 & 1.222 & 1.183 & 2.817 & 7.365 & 3.291 & 5.961 \\
\hline 16.104 & 1.974 & 14.130 & 1.919 & 14.122 & 10.520 & 1.230 & 1.150 & 10.402 & 1.192 & 1.103 & 2.740 & 7.662 & 3.538 & 5.204 \\
\hline 16.433 & 1.945 & 14.488 & 1.890 & 14.480 & 10.930 & 1.150 & 1.140 & 10.812 & 1.112 & 1.093 & 2.594 & 8.218 & 3.796 & 4.826 \\
\hline 16.786 & 1.907 & 14.879 & 1.852 & 14.871 & 11.200 & 1.080 & 1.080 & 11.082 & 1.042 & 1.033 & 2.451 & 8.631 & - & - \\
\hline 16.952 & 1.888 & 15.064 & 1.833 & 15.056 & 11.240 & 1.100 & 1.070 & 11.122 & 1.062 & 1.023 & 2.484 & 8.638 & - & - \\
\hline 17.112 & 1.868 & 15.244 & 1.813 & 15.236 & 11.440 & 1.060 & 1.050 & 11.322 & 1.022 & 1.003 & 2.406 & 8.916 & - & - \\
\hline 17.271 & 1.844 & 15.427 & 1.789 & 15.419 & 12.094 & 1.007 & 1.016 & 11.976 & 0.969 & 0.969 & 2.301 & 9.675 & - & - \\
\hline 17.420 & 1.814 & 15.606 & 1.759 & 15.598 & 12.110 & 1.006 & 1.029 & 11.992 & 0.968 & 0.982 & 2.303 & 9.689 & - & - \\
\hline & & M71 & & & 12.230 & 0.993 & 1.002 & 12.112 & 0.955 & 0.955 & 2.272 & 9.840 & - & - \\
\hline 11.997 & 5.401 & 6.596 & 4.628 & 6.490 & 12.712 & 0.913 & 0.966 & 12.594 & 0.875 & 0.919 & 2.118 & 10.476 & - & - \\
\hline 12.035 & 4.883 & 7.152 & 4.110 & 7.046 & 12.862 & 0.941 & 0.981 & 12.744 & 0.903 & 0.934 & 2.173 & 10.571 & - & - \\
\hline 12.116 & 4.552 & 7.564 & 3.779 & 7.458 & 12.934 & 0.917 & 0.972 & 12.816 & 0.879 & 0.925 & 2.127 & 10.689 & - & - \\
\hline 12.212 & 4.350 & 7.862 & 3.577 & 7.756 & 12.934 & 0.919 & 0.940 & 12.816 & 0.881 & 0.893 & 2.121 & 10.695 & - & - \\
\hline
\end{tabular}

Note. The last two columns for the cluster M67 indicate the $\left(V-K_{\mathrm{s}}\right)_{0}$ colours and $K_{\mathrm{s}_{0}}$ magnitudes of the bins used to draw the diagram of M67 in Figure 2. 
Table 6. Comparison of the Selective and Total Absorptions Evaluated by Using Different Extinction and Colour Excess Ratios

\begin{tabular}{|c|c|c|c|c|c|c|c|}
\hline $\begin{array}{l}\text { (1) } \\
\text { Cluster }\end{array}$ & $\begin{array}{c}(2) \\
E(B-V)\end{array}$ & $\begin{array}{c}(3) \\
E\left(V-K_{\mathrm{s}}\right)_{p}\end{array}$ & $\begin{array}{c}(4) \\
E\left(V-K_{\mathrm{s}}\right)_{c}\end{array}$ & $\begin{array}{c}(5) \\
\Delta E\left(V-K_{\mathrm{s}}\right)\end{array}$ & $\begin{array}{c}(6) \\
\left(A_{K_{\mathrm{s}}}\right)_{p}\end{array}$ & $\begin{array}{c}(7) \\
\left(A_{K_{\mathrm{s}}}\right)_{c}\end{array}$ & $\begin{array}{c}(8) \\
\Delta A_{K_{\mathrm{s}}}\end{array}$ \\
\hline M92 & 0.025 & 0.068 & 0.069 & 0.001 & 0.010 & 0.032 & 0.022 \\
\hline M13 & 0.020 & 0.054 & 0.055 & 0.001 & 0.008 & 0.025 & 0.018 \\
\hline M71 & 0.280 & 0.762 & 0.767 & 0.005 & 0.106 & 0.353 & 0.246 \\
\hline M67 & 0.038 & 0.103 & 0.104 & 0.001 & 0.014 & 0.048 & 0.033 \\
\hline NGC 6791 & 0.150 & 0.408 & 0.411 & 0.003 & 0.057 & 0.189 & 0.132 \\
\hline
\end{tabular}

Notes. Description of columns: (1) the cluster; (2) adopted $E(B-V)$ colour excess; (3) $E\left(V-K_{\mathrm{s}}\right)_{p}$, the colour excess evaluated by the equation $E\left(V-K_{\mathrm{s}}\right) / E(B-V)=2.72$ used in the paper; (4) $E\left(V-K_{\mathrm{s}}\right)$, the colour excess evaluated by the equation $E(V-$ $\left.K_{\mathrm{s}}\right) / E(B-V)=2.74$ for comparison purposes; (5) $\Delta E\left(V-K_{\mathrm{s}}\right)$, the difference between the colour excesses in columns (3) and (4); (6) $\left(A_{K_{\mathrm{s}}}\right)_{p}$, the total absorption evaluated by the equation $A_{K_{\mathrm{s}}} / E(B-V)=0.38$ used in the paper; (7) $\left(A_{K_{\mathrm{s}}}\right)_{c}$, the total absorption evaluated by the equation $A_{K_{\mathrm{s}}} / E(B-V)=1.26$; and (8) $\Delta A_{K_{\mathrm{s}}}{ }_{\mathrm{s}}$, the difference between the total absorptions in columns (6) and (7).

Table 7. Numerical Values of the Coefficients $b_{i}(i=1,2,3,4,5)$ in Equation (5)

\begin{tabular}{lcccrr}
\hline \hline $\begin{array}{l}\text { Cluster } \rightarrow \\
\left(V-K_{\mathrm{s}}\right)_{0} \rightarrow\end{array}$ & M92 & M13 & M71* & M67 & NGC 6791 \\
\hline$b_{5}$ & {$[1.76,3.04]$} & {$[1.76,3.69]$} & {$[1.79,3.10]$} & {$[2.14,3.80]$} & {$[2.44,4.13]$} \\
$b_{4}$ & - & - & - & 1.5121 & - \\
$b_{3}$ & 2.2478 & 0.9441 & 0.3976 & -21.290 & 1.2082 \\
$b_{2}$ & -22.7640 & -11.2930 & -5.6464 & 117.700 & -16.8610 \\
$b_{1}$ & 87.9930 & 51.2230 & 30.0540 & -317.680 & 88.1870 \\
$b_{0}$ & -157.9500 & -106.9700 & -72.7860 & 412.050 & -207.2100 \\
\hline \hline
\end{tabular}

${ }^{*}$ The $\left(V-K_{\mathrm{s}}\right)_{0}$ domain of the cluster M71 is in fact $[1.79,4.63]$. However, we restricted it with an upper limit of 3.10 mag due to some uncertainties claimed by the authors.

0.295, $E\left(H-K_{\mathrm{s}}\right) / E(J-H)=0.49$ (Turner 2011), and evaluated the corresponding $E\left(V-K_{\mathrm{s}}\right)=2.74 E(B-V)$ selective and $A_{K_{\mathrm{s}}}=1.26 E(B-V)$ total absorptions for the clusters. The results are given in Table 6. The differences in $E\left(V-K_{\mathrm{s}}\right)$ and $\Delta A_{K_{\mathrm{s}}}$ are almost the same as in Table 2. We prefer the extinction ratio and the colour excess ratios used in our study due to the reason explained in Section 2.1.

We fitted the $\left(V-K_{\mathrm{s}}\right)_{0}$ colours and $K_{\mathrm{s} 0}$ magnitudes to a fourth-degree polynomial for all clusters, except M67 for which a fifth-degree polynomial provided a higher correlation coefficient. The calibration of $K_{\mathrm{s}_{0}}$ is as follows:

$$
K_{\mathrm{s}_{0}}=\sum_{i=0}^{5} b_{i}\left(V-K_{\mathrm{s}}\right)_{0}^{i} .
$$

The numerical values of the coefficients $b_{i}(i=1,2,3,4$, $5)$ are given in Table 7 and the corresponding diagrams are presented in Figure 2. The $\left(V-K_{\mathrm{s}}\right)_{0}$ interval in the second line of the table indicates the range of $\left(V-K_{\mathrm{s}}\right)_{0}$ available for each cluster.

\section{THE PROCEDURE}

\subsection{Absolute Magnitude as a Function of Metallicity}

We adopted the procedure in Paper II, which consists of calibration of an absolute magnitude as a function of metallicity.
We calibrated the $M_{J}$ and $M_{K}$, absolute magnitudes in terms of metallicity for a given $(V-J)_{0}$ and $\left(V-K_{\mathrm{s}}\right)_{0}$ colour, respectively.

\subsubsection{Calibration of $M_{J}$ in Terms of Metallicity}

We estimated the $M_{J}$ absolute magnitudes for the $(V-J)_{0}$ colours given in Table 8 for the clusters M92, M13, M71, M67, and NGC 6791 by combining the $J_{0}$ apparent magnitudes evaluated by using Equation (2) and the true distance modulus $\left(\mu_{0}\right)$ of the cluster in question, i.e.

$$
M_{J}=J_{0}-\mu_{0} .
$$

Then, we plotted the absolute magnitudes versus $(V-J)_{0}$ colours. Figure 3 shows that the absolute magnitude is metallicity dependent. It increases (algebraically) with increasing metallicity and decreasing colour.

Now, we can fit the $M_{J}$ absolute magnitudes to the corresponding $[\mathrm{Fe} / \mathrm{H}]$ metallicity for a given $(V-J)_{0}$ colour index and obtain the required calibration. This is carried out for the colour indices $(V-J)_{0}=1.30,1.60,1.90,2.10,2.25,2.50$, and $2.65 \mathrm{mag}$ just for exhibition of the procedure. The results are given in Table 9 and Figure 4. The absolute magnitudes in all colour indices could be fitted to quadratic polynomials with high (squared) correlation coefficients, i.e. $R^{2} \geq 0.9982$, including $(V-J)_{0}=1.90,2.10$, and $2.25 \mathrm{mag}$, which cover the largest metallicity range, $-2.15 \leq[\mathrm{Fe} / \mathrm{H}] \leq+0.37$ dex. A high correlation coefficient plus (relatively) polynomial 


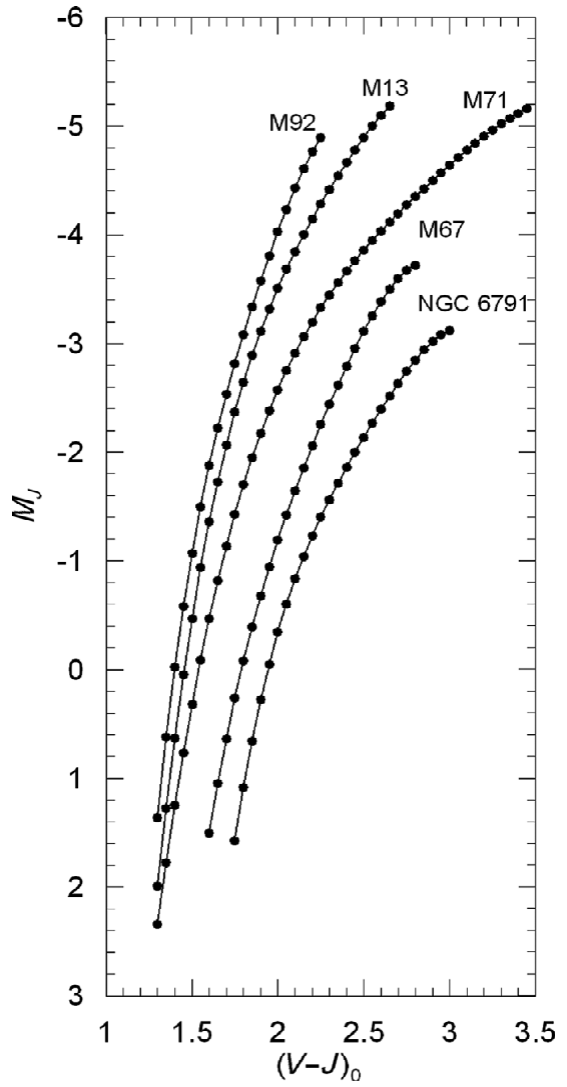

Figure 3. $M_{J} \times(V-J)_{0}$ colour-absolute magnitude diagrams for five clusters used for the absolute magnitude calibration.

with small degree is a strong clue for accurate absolute magnitude estimation.

This procedure can be applied to any $(V-J)_{0}$ colour interval for which the sample clusters are defined. The $(V-J)_{0}$ domain of the clusters is different. Hence, we adopted this interval as $1.3 \leq(V-J)_{0} \leq 2.8 \mathrm{mag}$, where at least two clusters are defined, and we evaluated $M_{J}$ absolute magnitude for each colour. Here, $1.72 \leq(V-J)_{0} \leq 2.28 \mathrm{mag}$ is the interval where all the clusters are defined, whereas the range of the interval where only two clusters (M67 and NGC 6791) are defined is rather small, i.e. $2.69 \leq(V-J)_{0} \leq 2.80$ mag. However, this small interval can be used for estimation of the absolute magnitudes for red giants with metallicities $-0.04 \leq[\mathrm{Fe} / \mathrm{H}] \leq+0.37 \mathrm{dex}$. The general form of the equation for the calibration is as follows:

$$
M_{J}=c_{0}+c_{1} X+c_{2} X^{2},
$$

where $X=[\mathrm{Fe} / \mathrm{H}] . M_{J}$ could be fitted in terms of metallicity by a quadratic polynomial for all $(V-J)_{0}$ colour intervals with a high correlation coefficient, $R^{2} \geq 0.996$, except two intervals with small ranges, i.e. a linear fitting was sufficient for the colour intervals $1.34 \leq(V-J)_{0} \leq 1.41$ and $2.69 \leq$ $(V-J)_{0} \leq 2.80$ mag where only two clusters are defined. The absolute magnitudes estimated via Equation (7) for 151 $(V-J)_{0}$ colour indices and the corresponding $c_{i}(i=0,1,2)$ coefficients are given in Table 10. However, the diagrams for
Table 8. $M_{J}$ Absolute Magnitudes Estimated for a Set of $(V-J)_{0}$ Colours for Five Clusters Used in the Calibration

\begin{tabular}{|c|c|c|c|c|c|}
\hline \multirow{2}{*}{$\begin{array}{l}\text { Cluster } \rightarrow \\
(V-J)_{0}\end{array}$} & M92 & M13 & M71 & M67 & NGC 6791 \\
\hline & \multicolumn{5}{|c|}{$M_{J}$} \\
\hline 1.30 & 1.364 & 1.992 & 2.341 & - & - \\
\hline 1.35 & 0.620 & 1.275 & 1.774 & - & - \\
\hline 1.40 & -0.025 & 0.629 & 1.251 & - & - \\
\hline 1.45 & -0.584 & 0.049 & 0.767 & - & - \\
\hline 1.50 & -1.073 & -0.472 & 0.321 & - & - \\
\hline 1.55 & -1.501 & -0.938 & -0.091 & - & - \\
\hline 1.60 & -1.881 & -1.356 & -0.469 & 1.505 & - \\
\hline 1.65 & -2.222 & -1.731 & -0.817 & 1.048 & - \\
\hline 1.70 & -2.532 & -2.068 & -1.137 & 0.635 & - \\
\hline 1.75 & -2.817 & -2.370 & -1.431 & 0.261 & 1.571 \\
\hline 1.80 & -3.084 & -2.643 & -1.700 & -0.080 & 1.083 \\
\hline 1.85 & -3.336 & -2.890 & -1.948 & -0.391 & 0.655 \\
\hline 1.90 & -3.576 & -3.114 & -2.175 & -0.678 & 0.278 \\
\hline 1.95 & -3.806 & -3.320 & -2.383 & -0.944 & -0.053 \\
\hline 2.00 & -4.026 & -3.509 & -2.574 & -1.192 & -0.345 \\
\hline 2.05 & -4.235 & -3.684 & -2.750 & -1.425 & -0.604 \\
\hline 2.10 & -4.431 & -3.847 & -2.912 & -1.646 & -0.835 \\
\hline 2.15 & -4.609 & -4.001 & -3.061 & -1.857 & -1.042 \\
\hline 2.20 & -4.766 & -4.146 & -3.199 & -2.058 & -1.231 \\
\hline 2.25 & -4.894 & -4.285 & -3.327 & -2.253 & -1.404 \\
\hline 2.30 & - & -4.417 & -3.447 & -2.439 & -1.566 \\
\hline 2.35 & - & -4.545 & -3.559 & -2.619 & -1.717 \\
\hline 2.40 & - & -4.667 & -3.664 & -2.792 & -1.862 \\
\hline 2.45 & - & -4.783 & -3.763 & -2.956 & -2.001 \\
\hline 2.50 & - & -4.895 & -3.857 & -3.111 & -2.135 \\
\hline 2.55 & - & -5.000 & -3.947 & -3.255 & -2.266 \\
\hline 2.60 & - & -5.097 & -4.033 & -3.386 & -2.393 \\
\hline 2.65 & - & -5.186 & -4.116 & -3.501 & -2.516 \\
\hline 2.70 & - & - & -4.197 & -3.598 & -2.634 \\
\hline 2.75 & - & - & -4.275 & -3.672 & -2.745 \\
\hline 2.80 & - & - & -4.352 & -3.719 & -2.848 \\
\hline 2.85 & - & - & -4.427 & - & -2.940 \\
\hline 2.90 & - & - & -4.500 & - & -3.019 \\
\hline 2.95 & - & - & -4.572 & - & -3.080 \\
\hline 3.00 & - & - & -4.642 & - & -3.120 \\
\hline 3.05 & - & - & -4.711 & - & - \\
\hline 3.10 & - & - & -4.778 & - & - \\
\hline 3.15 & - & - & -4.843 & - & - \\
\hline 3.20 & - & - & -4.905 & - & - \\
\hline 3.25 & - & - & -4.965 & - & - \\
\hline 3.30 & - & - & -5.021 & - & - \\
\hline 3.35 & - & - & -5.073 & - & - \\
\hline 3.40 & - & - & -5.120 & - & - \\
\hline 3.45 & - & - & -5.162 & - & - \\
\hline
\end{tabular}

the calibrations are not given in the paper because of space constraints.

\subsubsection{Calibration of $M_{K_{s}}$ in Terms of Metallicity}

We estimated the $M_{K}$ absolute magnitudes for the $\left(V-K_{\mathrm{s}}\right)_{0}$ colours given in Table 11 for the clusters M92, M13, M71, M67, and NGC 6791 by combining the $K_{\mathrm{s}_{0}}$ apparent magnitudes evaluated by Equation (5) and the true distance modulus $\left(\mu_{0}\right)$ of the cluster in question, i.e.

$$
M_{K_{\mathrm{s}}}=K_{\mathrm{s}_{0}}-\mu_{0} .
$$


Table 9. $M_{J}$ Absolute Magnitudes and $[\mathrm{Fe} / \mathrm{H}]$ Metallicities for Seven $(V-J)_{0}$ Intervals

\begin{tabular}{lrr}
\hline \hline$(V-J)_{0}$ & {$[\mathrm{Fe} / \mathrm{H}](\mathrm{dex})$} & $M_{J}$ \\
\hline 1.30 & -2.15 & 1.364 \\
& -1.41 & 1.992 \\
& -0.78 & 2.341 \\
1.60 & -2.15 & -1.881 \\
& -1.41 & -1.356 \\
& -0.78 & -0.469 \\
& -0.04 & 1.505 \\
1.90 & -2.15 & -3.576 \\
& -1.41 & -3.114 \\
& -0.78 & -2.175 \\
& -0.04 & -0.678 \\
2.10 & 0.37 & 0.278 \\
& -2.15 & -4.431 \\
& -1.41 & -3.847 \\
& -0.78 & -2.912 \\
2.25 & -0.04 & -1.646 \\
& 0.37 & -0.835 \\
& -2.15 & -4.894 \\
& -1.41 & -4.285 \\
& -0.78 & -3.327 \\
& -0.04 & -2.253 \\
& 0.37 & -1.404 \\
& -1.41 & -4.895 \\
& -0.04 & -3.111 \\
& 0.37 & -2.135 \\
& -1.41 & -5.186 \\
& -0.04 & -3.501 \\
& 0.37 & -2.516 \\
\hline & &
\end{tabular}

Then, we plotted the absolute magnitudes versus $\left(V-K_{\mathrm{s}}\right)_{0}$ colours. Figure 5 shows a similar trend of Figure 3, i.e. the absolute magnitude increases (algebraically) with increasing metallicity and decreasing colour.

We fitted the $M_{K_{\mathrm{s}}}$ absolute magnitudes to the corresponding $[\mathrm{Fe} / \mathrm{H}]$ metallicity for the colour indices $\left(V-K_{\mathrm{s}}\right)_{0}=$ $1.80,2.00,2.20,2.40,2.60,3.00$, and $3.30 \mathrm{mag}$ just for the exhibition of the procedure. The results are given in Table 12 and Figure 6. We can extend this fitting to a larger $\left(V-K_{\mathrm{s}}\right)_{0}$ interval for which the sample clusters are defined. The $\left(V-K_{\mathrm{s}}\right)_{0}$ domains of the clusters are different. Hence, we adopted this interval as $1.75 \leq\left(V-K_{\mathrm{s}}\right)_{0} \leq$ $3.80 \mathrm{mag}$ where at least two clusters are defined. However, the range of the interval where only two clusters are defined is rather limited, i.e. $1.75 \leq\left(V-K_{\mathrm{s}}\right)_{0} \leq 1.78$ and $3.70 \leq\left(V-K_{\mathrm{s}}\right)_{0} \leq 3.80 \mathrm{mag}$. The common domain of all the clusters is $2.45 \leq\left(V-K_{\mathrm{s}}\right)_{0} \leq 3.04 \mathrm{mag}$. The general form of the equation for the calibration is as follows:

$$
M_{K_{\mathrm{s}}}=d_{0}+d_{1} X+d_{2} X^{2},
$$

where $X=[\mathrm{Fe} / \mathrm{H}]$. Note that $M_{K_{\mathrm{s}}}$ could be fitted in terms of metallicity by a quadratic polynomial for all $\left(V-K_{\mathrm{s}}\right)_{0}$ colour intervals with a high correlation coefficient, $R^{2} \geq$ 0.9972 , except two intervals with small ranges, i.e. a linear fitting was sufficient for the colour intervals $1.75 \leq$

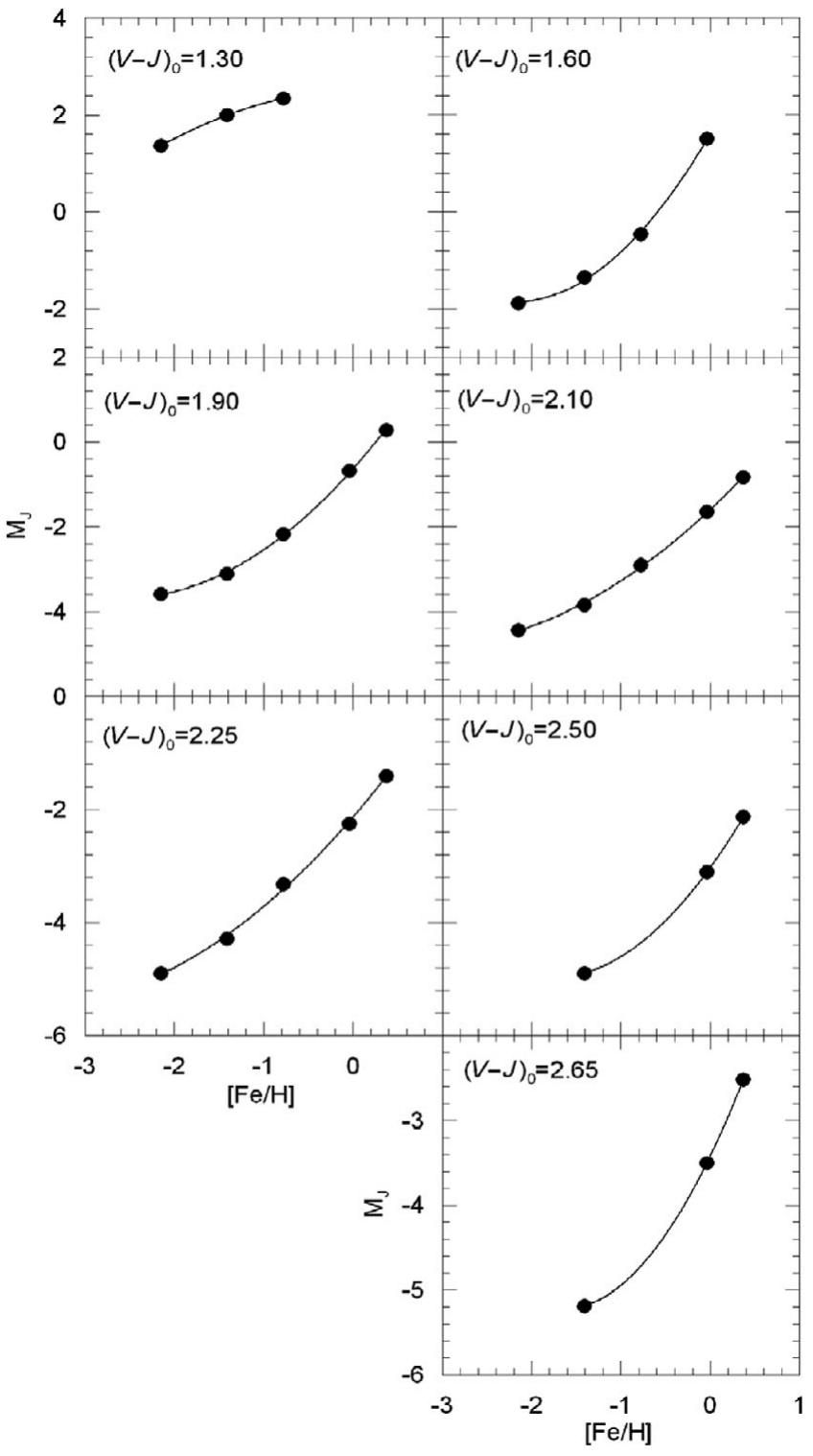

Figure 4. Calibration of the absolute magnitude $M_{J}$ as a function of metallicity $[\mathrm{Fe} / \mathrm{H}]$ for seven colour indices.

$\left(V-K_{\mathrm{s}}\right)_{0} \leq 1.78$ and $3.70 \leq\left(V-K_{\mathrm{s}}\right)_{0} \leq 3.80$ mag where only two clusters are defined. The absolute magnitudes estimated via Equation (9) for $206\left(V-K_{\mathrm{s}}\right)_{0}$ colour indices and the corresponding $d_{i}(i=0,1,2)$ coefficients are given in Table 13. However, the diagrams for the calibrations are not given here because of space constraints.

The calibration of the absolute magnitude $M_{K_{\mathrm{s}}}$ (and $M_{J}$ ) in terms of $[\mathrm{Fe} / \mathrm{H}]$ is carried out in steps of $0.01 \mathrm{mag}$. A small step is necessary to isolate an observational error on the colour $V-K_{\mathrm{s}}$ (and $V-J$ ) plus an error due to reddening. The origin of the errors mentioned is the trend of the RGB. Although they are not as steep as in $B V$ and $g r$ photometry, a small error in $V-K_{\mathrm{s}}$ (and $V-J$ ) implies a large change in the absolute magnitude. 
Table 10. $M_{J}$ Absolute Magnitudes Estimated for Five Galactic Clusters and the Numerical Values of $c_{i}(i=0,1,2)$ Coefficients in Equation $(7)$

\begin{tabular}{|c|c|c|c|c|c|c|c|c|c|c|c|c|c|c|c|c|c|c|c|c|c|}
\hline \multirow{2}{*}{$\begin{array}{l}\text { Cluster } \rightarrow \\
(V-J)_{0}\end{array}$} & M92 & M13 & M71 & M67 & NGC 6791 & \multirow[b]{2}{*}{$c_{0}$} & \multirow[b]{2}{*}{$c_{1}$} & \multirow[b]{2}{*}{$c_{2}$} & \multirow[b]{2}{*}{$R^{2}$} & \multirow[b]{2}{*}[\mathrm{Fe}/\mathrm{H}]{ Int. } & Cluster $\rightarrow$ & \multirow[t]{2}{*}{ M92 } & \multirow[t]{2}{*}{ M13 } & \multirow{2}{*}{$\begin{array}{r}\mathrm{M} 71 \\
M_{J}\end{array}$} & \multirow[t]{2}{*}{ M67 } & \multirow[t]{2}{*}{ NGC 6791} & \multirow[b]{2}{*}{$c_{0}$} & \multirow[b]{2}{*}{$c_{1}$} & \multirow[b]{2}{*}{$c_{2}$} & \multirow[b]{2}{*}{$R^{2}$} & \\
\hline & & & $M_{J}$ & & & & & & & & $(V-J)_{0}$ & & & & & & & & & & {$[\mathrm{Fe} / \mathrm{H}]$ Int. } \\
\hline 1.30 & 1.364 & 1.992 & 2.341 & - & - & 2.5339 & 0.0791 & -0.2162 & 1 & {$[-2.15,-0.78]$} & 2.06 & -4.275 & -3.717 & -2.783 & -1.470 & -0.652 & -1.4238 & 2.0438 & 0.3285 & 0.9991 & {$[-2.15,0.37]$} \\
\hline 1.31 & 1.207 & 1.843 & 2.224 & - & - & 2.4912 & 0.1978 & -0.1859 & 1 & {$[-2.15,-0.78]$} & 2.07 & -4.315 & -3.750 & -2.816 & -1.515 & -0.699 & -1.4661 & 2.0284 & 0.3220 & 0.9991 & {$[-2$.} \\
\hline 1.32 & 1.054 & 1.696 & 2.109 & - & - & 2.4485 & 0.3140 & -0.1557 & 1 & {$[-2.15,-0.78]$} & 2.08 & -4.354 & -3.783 & -2.849 & -1.559 & -0.745 & -1.5077 & 2.0134 & 0.3156 & 0.9992 & {$[-2.15,0.37]$} \\
\hline 1.33 & 0.905 & 1.553 & 1.996 & - & - & 2.4057 & 0.4277 & -0.1257 & 1 & {$[-2.15,-0.78]$} & 2.09 & -4.393 & -3.815 & -2.880 & -1.603 & -0.790 & -1.5486 & 1.9990 & 0.3095 & 0.9992 & {$[-2.15$} \\
\hline 1.34 & 0.760 & 1.412 & 1.884 & - & - & 2.5413 & 0.8220 & - & 0.9979 & {$[-2.15,-0.78]$} & 2.10 & -4.431 & -3.847 & -2.912 & -1.646 & -0.835 & -1.5888 & 1.9851 & 0.3037 & 0.9992 & {$[-2.15,0.37]$} \\
\hline 1.35 & 0.620 & 1.275 & 1.774 & - & - & 2.4436 & 0.8438 & - & 0.9990 & {$[-2.15,-0.78]$} & 2.11 & -4.468 & -3.879 & -2.943 & -1.689 & -0.878 & -1.6284 & 1.9717 & 0.2981 & 0.9992 & {$[-2.15,0.37]$} \\
\hline 1.36 & 0.484 & 1.140 & 1.666 & - & - & 2.3464 & 0.8639 & - & 0.9997 & {$[-2.15,-0.78]$} & 2.12 & -4.504 & -3.910 & -2.973 & -1.731 & -0.920 & -1.6675 & 1.9587 & 0.2927 & 0.9992 & {$[-2.15,0.37]$} \\
\hline 1.37 & 0.351 & 1.008 & 1.560 & - & - & 2.2497 & 0.8825 & - & 1 & {$[-2.15,-0.78]$} & 2.13 & -4.540 & -3.940 & -3.003 & -1.773 & -0.962 & -1.7059 & 1.9463 & 0.2876 & 0.9992 & {$[-2.15,0.37]$} \\
\hline 1.38 & 0.222 & 0.879 & 1.455 & - & - & 2.1535 & 0.8995 & - & 0.9999 & {$[-2.15,-0.78]$} & 2.14 & -4.575 & -3.971 & -3.032 & -1.815 & -1.002 & -1.7439 & 1.9343 & 0.2828 & 0.9992 & {$[-2.15,0.37]$} \\
\hline 1.39 & 0.097 & 0.752 & 1.352 & - & - & 2.0579 & 0.9152 & - & 0.9996 & {$[-2.15,-0.78]$} & 2.15 & -4.609 & -4.001 & -3.061 & -1.857 & -1.042 & -1.7813 & 1.9228 & 0.2782 & 0.9992 & {$[-2.15,0.37]$} \\
\hline 1.40 & -0.025 & 0.629 & 1.251 & - & - & 1.9629 & 0.9294 & - & 0.9990 & {$[-2.15,-0.78]$} & 2.16 & -4.642 & -4.030 & -3.090 & -1.898 & -1.081 & -1.8182 & 1.9118 & 0.2740 & 0.9992 & {$[-2.15,0.37]$} \\
\hline 1.41 & -0.143 & 0.508 & 1.151 & - & - & 1.8686 & 0.9424 & - & 0.9982 & {$[-2.15,-0.78]$} & 2.17 & -4.675 & -4.060 & -3.118 & -1.938 & -1.120 & -1.8547 & 1.9012 & 0.2700 & 0.9991 & {$[-2.15,0.37]$} \\
\hline 1.42 & -0.258 & 0.389 & 1.053 & - & - & 2.0168 & 1.3377 & 0.1301 & 1 & {$[-2.15,-0.78]$} & 2.18 & -4.706 & -4.089 & -3.145 & -1.979 & -1.158 & -1.8907 & 1.8911 & 0.2664 & 0.9991 & {$[-2.15,0.37]$} \\
\hline 1.43 & -0.370 & 0.273 & 0.956 & - & - & 1.9728 & 1.4259 & 0.1564 & 1 & {$[-2.15,-0.78]$} & 2.19 & -4.736 & -4.118 & -3.172 & -2.019 & -1.195 & -1.9263 & 1.8815 & 0.2632 & 0.9990 & {$[-2.15,0.37]$} \\
\hline 1.44 & -0.478 & 0.160 & 0.861 & - & - & 1.9286 & 1.5114 & 0.1823 & 1 & {$[-2.15,-0.78]$} & 2.20 & -4.766 & -4.146 & -3.199 & -2.058 & -1.2 & -1.9615 & 1.8724 & 0.2603 & 0.9990 & {$[-2.15,0.37]$} \\
\hline 1.45 & -0.584 & 0.049 & 0.767 & - & - & 1.8843 & 1.5943 & 0.2075 & 1 & {$[-2.15,-0.7$} & & -4.7 & -4.175 & -3.2 & -2.0 & -1.2 & -1.9 & 1.8638 & 0.2578 & 0.9989 & {$[-2.15,0.37]$} \\
\hline 1.46 & -0.687 & -0.060 & 0.675 & - & - & 397 & 1.6746 & 0.2322 & 1 & {$[-2$.} & 2.22 & -4.8 & -4.2 & -3.2 & -2.137 & -1 . & -2.0 & 1.8857 & 0.2557 & 0.9987 & {$[-2.15,0.37]$} \\
\hline 1.47 & -0.787 & -0.166 & 0.584 & - & - & 1.7949 & 1.7523 & 0.2564 & 1 & {$[-2.15,-0.78]$} & 2.23 & -4.846 & -4.230 & -3.277 & -2.176 & -1.337 & -2.0649 & 1.8480 & 0.2540 & 0.9986 & {$[-2.15,0.37]$} \\
\hline 1.48 & -0.885 & -0.270 & 0.495 & - & - & 1.7498 & 1.8273 & 0.2799 & 1 & {$[-2.15,-0.78]$} & 2.24 & -4.871 & -4.258 & -3.303 & -2.214 & -1.371 & -2.0987 & 1.8409 & 0.2527 & 0.9985 & {$[-2.15,0.37]$} \\
\hline 1.49 & -0.980 & -0.372 & 0.407 & - & - & 1.7046 & 1.8997 & 0.3028 & 1 & {$[-2.15,-0.78]$} & 2.25 & -4.894 & -4.285 & -3.327 & -2.253 & -1.404 & -2.1322 & 1.8343 & 0.2519 & 0.9983 & {$[-2.15,0.37]$} \\
\hline 1.50 & -1.073 & -0.472 & 0.321 & - & - & 1.6590 & 1.9694 & 0.3251 & 1 & {$[-2.15,-0.78]$} & 2.26 & -4.915 & -4.312 & -3.352 & -2.290 & -1.437 & -2.1654 & 1.8282 & 0.2516 & 0.9981 & {$[-2.15,0.37]$} \\
\hline 1.51 & -1.163 & -0.569 & 0.236 & - & - & 1.6133 & 2.0366 & 0.3467 & 1 & {$[-2.15,-0.78]$} & 2.27 & -4.935 & -4.339 & -3.376 & -2.328 & -1.470 & -2.1984 & 1.8226 & 0.2517 & 0.9979 & {$[-2.15,0.37]$} \\
\hline 1.52 & -1.251 & -0.664 & 0.152 & - & - & 1.5673 & 2.1011 & 0.3677 & 1 & {$[-2.15,-0.78]$} & 2.28 & -4.954 & -4.365 & -3.400 & -2.365 & -1.502 & -2.2311 & 1.8176 & 0.2524 & 0.9976 & {$[-2.15,0.37]$} \\
\hline 1.53 & -1.336 & -0.758 & 0.070 & - & - & 1.5210 & 2.1630 & 0.3879 & 1 & {$[-2.15,-0.78]$} & 2.29 & - & -4.391 & -3.424 & -2.403 & -1.534 & -2.2417 & 1.7305 & 0.1585 & 0.9965 & {$[-1.41,0.37]$} \\
\hline 1.54 & -1.420 & -0.849 & -0.011 & - & - & 1.4745 & 2.2223 & 0.4075 & 1 & {$[-2.15,-0.78]$} & 2.30 & - & -4.417 & -3.447 & -2.439 & -1.566 & -2.2734 & 1.7244 & 0.1577 & 0.9960 & {$[-1.41,0.37]$} \\
\hline 1.55 & -1.501 & -0.938 & -0.091 & - & - & 1.4277 & 2.2791 & 0.4264 & 1 & {$[-2.15,-0.78]$} & 2.31 & - & -4.443 & - & -2.476 & -1.597 & -2.3960 & 2.0134 & 0.3982 & 1 & {$[-1.41,0.37]$} \\
\hline 1.56 & -1.581 & -1.025 & -0.169 & - & - & 1.3806 & 2.3332 & 0.4445 & 1 & {$[-2.15,-0.78]$} & 2.32 & - & -4.469 & - & -2.512 & -1.627 & -2.4319 & 2.0230 & 0.4102 & 1 & {$[-1.41,0.37]$} \\
\hline 1.57 & -1.659 & -1.111 & -0.246 & - & - & 1.3333 & 2.3849 & 0.4620 & 1 & {$[-2.15,-0.78]$} & 2.33 & - & -4.494 & - & -2.548 & -1.658 & -2.4675 & 2.0328 & 0.4222 & 1 & {$[-1.41,0.37]$} \\
\hline 1.58 & -1.735 & -1.194 & -0.321 & 1.702 & - & 1.8084 & 3.2112 & 0.7317 & 0.9984 & {$[-2.15,-0.04]$} & 2.34 & - & -4.520 & - & -2.584 & -1.688 & -2.5029 & 2.0426 & 0.4342 & 1 & {$[-1.41,0.37]$} \\
\hline 1.59 & -1.809 & -1.276 & -0.396 & 1.603 & - & 1.7099 & 3.1824 & 0.7231 & 0.9987 & {$[-2.15,-0.04]$} & 2.35 & - & -4.545 & - & -2.619 & -1.717 & -2.5379 & 2.0525 & 0.4463 & 1 & {$[-1.41,0.37]$} \\
\hline 1.60 & -1.881 & -1.356 & -0.469 & 1.505 & - & 1.6134 & 3.1544 & 0.7148 & 0.9989 & {$[-2.15,-0.04]$} & 2.36 & - & -4.569 & - & -2.654 & -1.747 & -2.5726 & 2.0624 & 0.4583 & 1 & {$[-1.41,0.37]$} \\
\hline 1.61 & -1.952 & -1.435 & -0.541 & 1.410 & - & 1.5188 & 3.1272 & 0.7069 & 0.9991 & {$[-2.15,-0.04]$} & 2.37 & - & -4.594 & - & -2.689 & -1.776 & -2.6070 & 2.0723 & 0.4702 & 1 & {$[-1.41,0.37]$} \\
\hline 1.62 & -2.022 & -1.511 & -0.612 & 1.317 & - & 1.4262 & 3.1008 & 0.6992 & 0.9993 & {$[-2.15,-0.04]$} & 2.38 & - & -4.618 & - & -2.724 & -1.805 & -2.6412 & 2.0821 & 0.4821 & 1 & {$[-1.41,0.37]$} \\
\hline 1.63 & -2.090 & -1.586 & -0.682 & 1.225 & - & 1.3354 & 3.0752 & 0.6918 & 0.9995 & {$[-2.15,-0.04]$} & 2.39 & - & -4.643 & - & -2.758 & -1.833 & -2.6750 & 2.0917 & 0.4938 & 1 & {$[-1.41,0.37]$} \\
\hline 1.64 & -2.157 & -1.659 & -0.750 & 1.136 & - & 1.2464 & 3.0502 & 0.6846 & 0.9996 & {$[-2.15,-0.04]$} & 2.40 & - & -4.667 & - & -2.792 & -1.862 & -2.7085 & 2.1013 & 0.5053 & 1 & {$[-1.41,0.37]$} \\
\hline 1.65 & -2.222 & -1.731 & -0.817 & 1.048 & - & 1.1592 & 3.0258 & 0.6777 & 0.9997 & {$[-2.15,-0.04]$} & 2.41 & - & -4.690 & - & -2.825 & -1.890 & -2.7417 & 2.1106 & 0.5167 & 1 & {$[-1.41,0.37]$} \\
\hline 1.66 & -2.286 & -1.801 & -0.883 & 0.962 & - & 1.0737 & 3.0019 & 0.6709 & 0.9998 & {$[-2.15,-0.04]$} & 2.42 & - & -4.714 & - & -2.859 & -1.918 & -2.7746 & 2.1197 & 0.5278 & 1 & {$[-1.41,0.37]$} \\
\hline 1.67 & -2.349 & -1.870 & -0.948 & 0.878 & - & 0.9899 & 2.9786 & 0.6642 & 0.9999 & {$[-2.15,-0.04]$} & 2.43 & - & -4.737 & - & -2.891 & -1.946 & -2.8072 & 2.1286 & 0.5388 & 1 & {$[-1.41,0.37]$} \\
\hline 1.68 & -2.411 & -1.937 & -1.012 & 0.795 & - & 0.9078 & 2.9558 & 0.6577 & 0.9999 & {$[-2.15,-0.04]$} & 2.44 & - & -4.761 & - & -2.924 & -1.973 & -2.8394 & 2.1372 & 0.5494 & 1 & $-1.41,0.37]$ \\
\hline 1.69 & -2.472 & -2.003 & -1.075 & 0.714 & - & 0.8272 & 2.9333 & 0.6513 & 1 & {$[-2.15,-0.04]$} & 2.45 & - & -4.783 & - & -2.956 & -2.001 & -2.8713 & 2.1454 & 0.5597 & 1 & {$[-1.41,0.37]$} \\
\hline 1.70 & -2.532 & -2.068 & -1.137 & 0.635 & - & 482 & 2.9113 & 0.6449 & 1 & {$[-2.15,-0.04]$} & 2.46 & - & -4.806 & - & -2.988 & -2.028 & -2.9028 & 2.1533 & 0.5697 & 1 & {$[-1.41,0.37]$} \\
\hline 1.71 & & -2.131 & -1.198 & 0.557 & - & 0.6706 & 2.8896 & 0.6387 & 1 & {$[-2.15,-0.04]$} & 2.47 & - & -4.829 & - & -3.019 & -2.055 & -2.9339 & 2.1608 & 0.5794 & 1 & {$[-1.41,0.37]$} \\
\hline 1.72 & -2.649 & -2.193 & -1.258 & 0.481 & 1.895 & 0.6564 & 3.0018 & 0.6839 & 0.9996 & {$[-2.15,0.37]$} & 2.48 & - & -4.851 & - & -3.050 & -2.082 & -2.9647 & 2.1678 & 0.5887 & 1 & {$[-1.41,0.37]$} \\
\hline
\end{tabular}


Table 10. Continued.

\begin{tabular}{|c|c|c|c|c|c|c|c|c|c|c|c|c|c|c|c|c|c|c|c|c|c|}
\hline \multirow{2}{*}{$\begin{array}{l}\text { Cluster } \rightarrow \\
(V-J)_{0}\end{array}$} & M92 & M13 & M71 & M67 & NGC 6791 & \multicolumn{6}{|r|}{ Cluster $\rightarrow$} & M92 & M13 & M71 & M67 & \multicolumn{6}{|l|}{ NGC 6791} \\
\hline & & & $M_{J}$ & & & $c_{0}$ & $c_{1}$ & $c_{2}$ & $R^{2}$ & {$[\mathrm{Fe} / \mathrm{H}]$ Int. } & $(V-J)_{0}$ & & & & & & $c_{0}$ & $c_{1}$ & $c_{2}$ & $R^{2}$ & {$[\mathrm{Fe} / \mathrm{H}] \mathrm{Int}$.} \\
\hline 1.73 & -2.706 & -2.253 & -1.316 & 0.406 & 1.784 & 0.5706 & 2.9565 & 0.6684 & 0.9997 & {$[-2.15,0.37]$} & 2.49 & - & -4.873 & - & -3.081 & -2.109 & -2.9950 & 2.1743 & 0.5975 & 1 & {$[-1.41,0.37]$} \\
\hline 1.74 & -2.762 & -2.312 & -1.374 & 0.333 & 1.676 & 0.4867 & 2.9127 & 0.6533 & 0.9998 & {$[-2.15,0.37]$} & 2.50 & - & -4.895 & - & -3.111 & -2.135 & -3.0250 & 2.1804 & 0.6059 & 1 & {$[-1.41,0.37]$} \\
\hline 1.75 & -2.817 & -2.370 & -1.431 & 0.261 & 1.571 & 0.4047 & 2.8703 & 0.6387 & 0.9999 & {$[-2.15,0.37]$} & 2.51 & - & -4.916 & - & -3.141 & -2.162 & -3.0546 & 2.1859 & 0.6139 & 1 & {$[-1.41,0.37]$} \\
\hline 1.76 & -2.872 & -2.427 & -1.487 & 0.190 & 1.468 & 0.3245 & 2.8291 & 0.6245 & 0.9999 & {$[-2.15,0.37]$} & 2.52 & - & -4.937 & - & -3.170 & -2.188 & -3.0837 & 2.1908 & 0.6213 & 1 & {$[-1.41,0.37]$} \\
\hline 1.77 & -2.926 & -2.483 & -1.541 & 0.121 & 1.368 & 0.2461 & 2.7893 & 0.6107 & 1 & {$[-2.15,0.37]$} & 2.53 & - & -4.958 & - & -3.199 & -2.214 & -3.1123 & 2.1951 & 0.6282 & 1 & {$[-1.41,0.37]$} \\
\hline 1.78 & -2.979 & -2.537 & -1.595 & 0.053 & 1.271 & 0.1695 & 2.7507 & 0.5973 & 1 & {$[-2.15,0.37]$} & 2.54 & - & -4.979 & - & -3.227 & -2.240 & -3.1405 & 2.1987 & 0.6346 & 1 & {$[-1.41,0.37]$} \\
\hline 1.79 & -3.032 & -2.591 & -1.648 & -0.014 & 1.176 & 0.0945 & 2.7133 & 0.5842 & 0.9999 & {$[-2.15,0.37]$} & 2.55 & - & -5.000 & - & -3.255 & -2.266 & -3.1683 & 2.2017 & 0.6404 & 1 & {$[-1.41,0.37]$} \\
\hline 1.80 & -3.084 & -2.643 & -1.700 & -0.080 & 1.083 & 0.0213 & 2.6771 & 0.5715 & 0.9999 & {$[-2.15,0.37]$} & 2.56 & - & -5.020 & - & -3.283 & -2.292 & -3.1955 & 2.2039 & 0.6455 & 1 & {$[-1.41,0.37]$} \\
\hline 1.81 & -3.135 & -2.694 & -1.752 & -0.144 & 0.993 & -0.0504 & 2.6420 & 0.5591 & 0.9999 & {$[-2.15,0.37]$} & 2.57 & - & -5.040 & - & -3.309 & -2.317 & -3.2222 & 2.2054 & 0.6500 & 1 & {$[-1.41,0.37]$} \\
\hline 1.82 & -3.186 & -2.745 & -1.802 & -0.208 & 0.905 & -0.1204 & 2.6080 & 0.5470 & 0.9998 & {$[-2.15,0.37]$} & 2.58 & - & -5.059 & - & -3.336 & -2.343 & -3.2484 & 2.2061 & 0.6539 & 1 & {$[-1.41,0.37]$} \\
\hline 1.83 & -3.237 & -2.794 & -1.851 & -0.270 & 0.819 & -0.1890 & 2.5750 & 0.5353 & 0.9998 & {$[-2.15,0.37]$} & 2.59 & - & -5.078 & - & -3.361 & -2.368 & -3.2740 & 2.2060 & 0.6570 & 1 & {$[-1.41,0.37]$} \\
\hline 1.84 & -3.286 & -2.843 & -1.900 & -0.331 & 0.736 & -0.2560 & 2.5431 & 0.5238 & 0.9997 & {$[-2.15,0.37]$} & 2.60 & - & -5.097 & - & -3.386 & -2.393 & -3.2990 & 2.2051 & 0.6594 & 1 & {$[-1.41,0.37]$} \\
\hline 1.85 & -3.336 & -2.890 & -1.948 & -0.391 & 0.655 & -0.3215 & 2.5122 & 0.5126 & 0.9996 & {$[-2.15,0.37]$} & 2.61 & - & -5.116 & - & -3.411 & -2.418 & -3.3235 & 2.2032 & 0.6611 & 1 & {$[-1.41,0.37]$} \\
\hline 1.86 & -3.385 & -2.937 & -1.995 & -0.450 & 0.576 & -0.3856 & 2.4822 & 0.5017 & 0.9996 & {$[-2.15,0.37]$} & 2.62 & - & -5.134 & - & -3.434 & -2.443 & -3.3473 & 2.2004 & 0.6620 & 1 & {$[-1.41,0.37]$} \\
\hline 1.87 & -3.433 & -2.982 & -2.041 & -0.509 & 0.498 & -0.4484 & 2.4532 & 0.4910 & 0.9995 & {$[-2.15,0.37]$} & 2.63 & - & -5.152 & - & -3.457 & -2.467 & -3.3705 & 2.1967 & 0.6621 & 1 & {$[-1.41,0.37]$} \\
\hline 1.88 & -3.481 & -3.027 & -2.086 & -0.566 & 0.423 & -0.5097 & 2.4251 & 0.4806 & 0.9994 & {$[-2.15,0.37]$} & 2.64 & - & -5.169 & - & -3.480 & -2.492 & -3.3931 & 2.1920 & 0.6613 & 1 & , 0.37] \\
\hline 1.89 & -3.529 & -3.071 & -2.131 & -0.622 & 0.350 & -0.5698 & 2.3978 & 0.4704 & 0.9994 & {$[-2.15,0.37]$} & 2.65 & - & -5.186 & - & -3.501 & -2.516 & -3.4149 & 2.1862 & 0.6597 & 1 & {$[-1.41,0.37]$} \\
\hline 1.90 & -3.576 & -3.114 & -2.175 & -0.678 & 0.278 & -0.6286 & 2.3713 & 0.4604 & 0.9993 & {$[-2.15,0.37]$} & 2.66 & - & -5.203 & - & -3.522 & -2.540 & -3.4361 & 2.1794 & 0.6572 & 1 & {$[-1.41,0.37]$} \\
\hline 1.91 & -3.623 & -3.157 & -2.218 & -0.733 & 0.209 & -0.6861 & 2.3457 & 0.4507 & 0.9992 & {$[-2.15,0.37]$} & 2.67 & - & -5.219 & - & -3.542 & -2.564 & -3.4565 & 2.1716 & 0.6537 & 1 & {$[-1.41,0.37]$} \\
\hline 1.92 & -3.669 & -3.199 & -2.260 & -0.786 & 0.141 & -0.7425 & 2.3209 & 0.4412 & 0.9992 & {$[-2.15,0.37]$} & 2.68 & - & -5.234 & - & -3.562 & -2.587 & -3.4761 & 2.1626 & 0.6494 & 1 & {$[-1.41,0.37]$} \\
\hline 1.93 & -3.715 & -3.240 & -2.302 & -0.840 & 0.075 & -0.7977 & 2.2968 & 0.4318 & 0.9991 & {$[-2.15,0.37]$} & 2.69 & - & - & - & -3.580 & -2.610 & 2.3650 & -3.4855 & - & 1 & {$[-0.04,0.37]$} \\
\hline 1.94 & -3.761 & -3.280 & -2.343 & -0.892 & 0.010 & -0.8517 & 2.2735 & 0.4227 & 0.9991 & {$[-2.15,0.37]$} & 2.70 & - & - & - & -3.598 & -2.634 & 2.3515 & -3.5036 & - & 1 & {$[-0.04,0.37]$} \\
\hline 1.95 & -3.806 & -3.320 & -2.383 & -0.944 & -0.053 & -0.9047 & 2.2509 & 0.4138 & 0.9991 & {$[-2.15,0.37]$} & 2.71 & - & - & - & -3.614 & -2.656 & 2.3366 & -3.5209 & - & 1 & {$[-0.04,0.37]$} \\
\hline 1.96 & -3.851 & -3.359 & -2.422 & -0.994 & -0.114 & -0.9565 & 2.2289 & 0.4051 & 0.9990 & {$[-2.15,0.37]$} & 2.72 & - & - & - & -3.630 & -2.679 & 2.3200 & -3.5374 & - & 1 & {$[-0.04,0.37]$} \\
\hline 1.97 & -3.895 & -3.397 & -2.461 & -1.045 & -0.174 & -1.0074 & 2.2077 & 0.3966 & 0.9990 & {$[-2.15,0.37]$} & 2.73 & - & - & - & -3.645 & -2.701 & 2.3018 & -3.5529 & - & 1 & {$[-0.04,0.37]$} \\
\hline 1.98 & -3.939 & -3.435 & -2.499 & -1.094 & -0.232 & -1.0572 & 2.1871 & 0.3882 & 0.9990 & {$[-2.15,0.37]$} & 2.74 & - & - & - & -3.659 & -2.723 & 2.2820 & -3.5676 & - & 1 & {$[-0.04,0.37]$} \\
\hline 1.99 & -3.983 & -3.472 & -2.537 & -1.143 & -0.289 & -1.1061 & 2.1671 & 0.3801 & 0.9990 & {$[-2.15,0.37]$} & 2.75 & - & - & - & -3.672 & -2.745 & 2.2604 & -3.5813 & - & 1 & {$[-0.04,0.37]$} \\
\hline 2.00 & -4.026 & -3.509 & -2.574 & -1.192 & -0.345 & -1.1541 & 2.1478 & 0.3721 & 0.9990 & {$[-2.15,0.37]$} & 2.76 & - & - & - & -3.683 & -2.766 & 2.2371 & -3.5940 & - & 1 & {$[-0.04,0.37]$} \\
\hline 2.01 & -4.069 & -3.545 & -2.610 & -1.239 & -0.399 & -1.2011 & 2.1290 & 0.3644 & 0.9990 & {$[-2.15,0.37]$} & 2.77 & - & - & - & -3.694 & -2.787 & 2.2120 & -3.6057 & - & 1 & {$[-0.04,0.37]$} \\
\hline 2.02 & -4.111 & -3.580 & -2.646 & -1.287 & -0.452 & -1.2473 & 2.1109 & 0.3568 & 0.9990 & {$[-2.15,0.37]$} & 2.78 & - & - & - & -3.704 & -2.808 & 2.1851 & -3.6164 & - & 1 & {$[-0.04,0.37]$} \\
\hline 2.03 & -4.153 & -3.615 & -2.681 & -1.333 & -0.504 & -1.2926 & 2.0933 & 0.3494 & 0.9991 & {$[-2.15,0.37]$} & 2.79 & - & - & - & -3.712 & -2.828 & 2.1564 & -3.6260 & - & 1 & {$[-0.04,0.37]$} \\
\hline 2.04 & -4.194 & -3.650 & -2.716 & -1.379 & -0.554 & -1.3371 & 2.0762 & 0.3423 & 0.9991 & {$[-2.15,0.37]$} & 2.80 & - & - & - & -3.719 & -2.848 & 2.1257 & -3.6344 & - & 1 & {$[-0.04,0.37]$} \\
\hline 2.05 & -4.235 & -3.684 & -2.750 & -1.425 & -0.604 & -1.3809 & 2.0598 & 0.3353 & 0.9991 & {$[-2.15,0.37]$} & - & - & - & - & - & - & - & - & - & - & - \\
\hline
\end{tabular}

Note. The 11th and 22nd columns give the range of the metallicity $[\mathrm{Fe} / \mathrm{H}](\mathrm{dex})$ for the star whose absolute magnitude would be estimated. $R^{2}$ is the square of the correlation coefficient. 
Table 11. $M_{K}$ Absolute Magnitudes Estimated for a Set of ( $V-$ $\left.K_{\mathrm{s}}\right)_{0}$ Colours for Five Clusters used in the Calibration

\begin{tabular}{|c|c|c|c|c|c|}
\hline \multirow{2}{*}{$\begin{array}{l}\text { Cluster } \rightarrow \\
\left(V-K_{\mathrm{s}}\right)_{0}\end{array}$} & M92 & M13 & M71 & M67 & NGC 6791 \\
\hline & \multicolumn{5}{|c|}{$M_{K_{\mathrm{s}}}$} \\
\hline 1.75 & 0.957 & 1.456 & - & - & - \\
\hline 1.80 & 0.434 & 0.919 & 1.742 & - & - \\
\hline 1.85 & -0.045 & 0.424 & 1.250 & - & - \\
\hline 1.90 & -0.485 & -0.031 & 0.792 & - & - \\
\hline 1.95 & -0.890 & -0.449 & 0.367 & - & - \\
\hline 2.00 & -1.265 & -0.834 & -0.028 & - & - \\
\hline 2.05 & -1.613 & -1.189 & -0.394 & - & - \\
\hline 2.10 & -1.938 & -1.515 & -0.733 & - & - \\
\hline 2.15 & -2.242 & -1.816 & -1.048 & 0.960 & - \\
\hline 2.20 & -2.529 & -2.094 & -1.339 & 0.635 & - \\
\hline 2.25 & -2.800 & -2.352 & -1.608 & 0.320 & - \\
\hline 2.30 & -3.059 & -2.591 & -1.857 & 0.016 & - \\
\hline 2.35 & -3.306 & -2.814 & -2.088 & -0.274 & - \\
\hline 2.40 & -3.543 & -3.023 & -2.302 & -0.550 & - \\
\hline 2.45 & -3.771 & -3.219 & -2.500 & -0.811 & 0.619 \\
\hline 2.50 & -3.992 & -3.403 & -2.683 & -1.057 & 0.256 \\
\hline 2.55 & -4.204 & -3.578 & -2.854 & -1.288 & -0.072 \\
\hline 2.60 & -4.408 & -3.745 & -3.012 & -1.505 & -0.369 \\
\hline 2.65 & -4.605 & -3.905 & -3.160 & -1.709 & -0.637 \\
\hline 2.70 & -4.793 & -4.058 & -3.299 & -1.901 & -0.880 \\
\hline 2.75 & -4.971 & -4.206 & -3.428 & -2.083 & -1.101 \\
\hline 2.80 & -5.138 & -4.350 & -3.550 & -2.255 & -1.302 \\
\hline 2.85 & -5.292 & -4.490 & -3.666 & -2.421 & -1.486 \\
\hline 2.90 & -5.432 & -4.626 & -3.776 & -2.581 & -1.656 \\
\hline 2.95 & -5.556 & -4.759 & -3.881 & -2.736 & -1.813 \\
\hline 3.00 & -5.659 & -4.890 & -3.981 & -2.890 & -1.960 \\
\hline 3.05 & -5.740 & -5.017 & -4.078 & -3.042 & -2.098 \\
\hline 3.10 & - & -5.142 & -4.172 & -3.194 & -2.230 \\
\hline 3.15 & - & -5.264 & & -3.347 & -2.357 \\
\hline 3.20 & - & -5.381 & - & -3.501 & -2.479 \\
\hline 3.30 & - & -5.604 & - & -3.815 & -2.717 \\
\hline 3.35 & - & -5.708 & - & -3.973 & -2.834 \\
\hline 3.40 & - & -5.805 & - & -4.130 & -2.951 \\
\hline 3.45 & - & -5.894 & - & -4.285 & -3.067 \\
\hline 3.50 & - & -5.975 & - & -4.435 & -3.184 \\
\hline 3.55 & - & -6.045 & - & -4.576 & -3.301 \\
\hline 3.60 & - & -6.104 & - & -4.705 & -3.418 \\
\hline 3.65 & - & -6.149 & - & -4.817 & -3.535 \\
\hline 3.70 & - & -6.179 & - & -4.905 & -3.651 \\
\hline 3.75 & - & - & - & -4.963 & -3.766 \\
\hline 3.80 & - & - & - & -4.984 & -3.878 \\
\hline 3.85 & - & - & - & - & -3.988 \\
\hline 3.90 & - & - & - & - & -4.092 \\
\hline 3.95 & - & - & - & - & -4.191 \\
\hline 4.00 & - & - & - & - & -4.283 \\
\hline 4.05 & - & - & - & - & -4.365 \\
\hline 4.10 & - & - & - & - & -4.436 \\
\hline
\end{tabular}

Iron abundance, $[\mathrm{Fe} / \mathrm{H}]$, is not the only parameter that determines the chemistry of the star, but $\alpha$ enhancement, $[\alpha / \mathrm{Fe}]$, is also equally important. However, as stated in Paper $\mathrm{I}$, there is a correlation between the two sets of abundances. Hence, we do not expect any considerable change in the numerical values of the estimated absolute magnitudes in the case of addition of the $\alpha$-enhancement term in Equations (7) and (9).

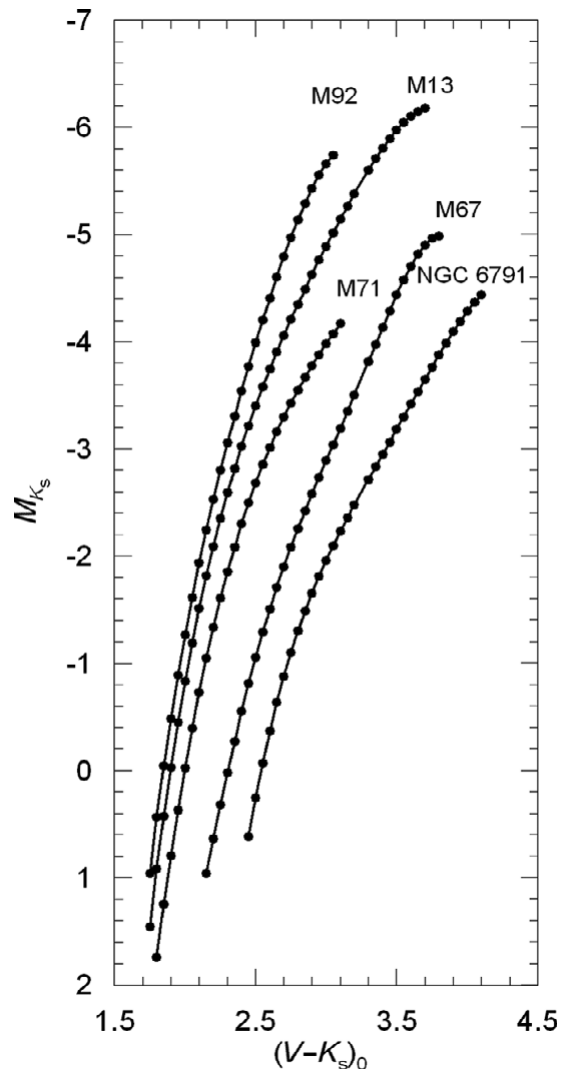

Figure 5. $M_{K_{\mathrm{s}}} \times\left(V-K_{\mathrm{s}}\right)_{0}$ colour-absolute magnitude diagrams for five clusters used for the absolute magnitude calibration.

\subsection{Application of the Method}

\subsubsection{Application of the $M_{J} \times[\mathrm{Fe} / \mathrm{H}]$ Calibration}

We applied the $M_{J} \times[\mathrm{Fe} / \mathrm{H}]$ calibration to the clusters M5 and M68. The reason for choosing clusters instead of field giants is that clusters provide absolute magnitudes for comparison with the ones estimated by means of our method. The $J$ magnitudes and $V-J$ colours for the cluster M5 are taken from Brasseur et al. (2010). 2MASS photometric data are not available for the cluster M68. Hence, the $J_{0}$ magnitudes and $(V-J)_{0}$ colours are provided by transformation of the $V, B-V$, and $V-I$ data of Walker (1994) to $J_{0}$ magnitudes and $(V-J)_{0}$ colours by Equation (1) and the procedure explained in Section 2.1. The data for the clusters are given in Table 14. Two references are given for the cluster M5. The colour excess $E(B-V)$ and the true distance modulus $\mu_{0}$ refer to the first author, whereas the metallicity which was tested in Paper I is taken from the second author. The $J_{0}$ magnitudes and $(V-J)_{0}$ colours as well as the original $V$, $B-V$, and $V-I$ data are given in Table 15 .

We evaluated the $M_{J}$ absolute magnitude by Equation (7) for a set of $(V-J)_{0}$ colour indices where the clusters are defined. The results are given in Table 16. The columns refer to (1) $(V-J)_{0}$ colour index; (2) $\left(M_{J}\right)_{\mathrm{cl}}$, the absolute magnitude for a cluster estimated by its colour-magnitude diagram; (3) $\left(M_{J}\right)_{\mathrm{ev}}$, the absolute magnitude estimated by the procedure; 
Table 12. $M_{K}$ Absolute Magnitudes and $[\mathrm{Fe} / \mathrm{H}]$ Metallicities for Seven $\left(V-K_{\mathrm{s}}^{\mathrm{s}}\right)_{0}$ Intervals

\begin{tabular}{lrr}
\hline \hline$\left(V-K_{\mathrm{s}}\right)_{0}$ & {$[\mathrm{Fe} / \mathrm{H}](\mathrm{dex})$} & $M_{K_{\mathrm{s}}}$ \\
\hline 1.80 & -2.15 & 0.434 \\
& -1.41 & 0.919 \\
& -0.78 & 1.742 \\
2.00 & -2.15 & -1.265 \\
& -1.41 & -0.834 \\
& -0.78 & -0.028 \\
2.20 & -2.15 & -2.529 \\
& -1.41 & -2.094 \\
& -0.78 & -1.339 \\
2.40 & -0.04 & 0.635 \\
& -2.15 & -3.543 \\
& -1.41 & -3.023 \\
& -0.78 & -2.302 \\
2.60 & -0.04 & -0.550 \\
& -2.15 & -4.408 \\
& -1.41 & -3.745 \\
& -0.78 & -3.012 \\
& -0.04 & -1.505 \\
3.30 & 0.37 & -0.369 \\
& -2.15 & -5.659 \\
& -1.41 & -4.890 \\
& -0.78 & -3.981 \\
& -0.04 & -2.890 \\
& 0.37 & -1.960 \\
& -1.41 & -5.604 \\
& -0.04 & -3.815 \\
& 0.37 & -2.717 \\
\hline & & \\
& &
\end{tabular}

(4) $\Delta M_{J}$, absolute magnitude residuals. Also, the metallicity for each cluster is indicated near the name of the cluster. The differences between the absolute magnitudes estimated by the procedure presented in this study and those evaluated via colour-magnitude diagrams of the clusters (the residuals) lie in a (relatively) short interval, i.e. -0.08 and $+0.34 \mathrm{mag}$, and the range of $94 \%$ of the absolute magnitude residuals is only $0<M_{J} \leq 0.3 \mathrm{mag}$. The mean and the standard deviation of (all) residuals are $\left\langle\Delta M_{J}\right\rangle=0.137$ and $\sigma_{M_{J}}=0.080$ mag, respectively. The distribution of the residuals are given in Table 17 and Figure 7.

\subsubsection{Application of the $M_{K_{\mathrm{s}}} \times[\mathrm{Fe} / \mathrm{H}]$ Calibration}

NGC 1851 is the last cluster in Brasseur et al. (2010) for which $K_{\mathrm{s}}$ magnitudes and $V-K_{\mathrm{s}}$ colours are available. However, the $M_{K_{\mathrm{s}}} \times\left(V-K_{\mathrm{s}}\right)_{0}$ colour-magnitude diagram of this cluster shows that the uncertainties in the data of this cluster are (relatively) large. Actually, the absolute magnitudes are fainter than the corresponding ones of the cluster M71 for the colour interval $\left(V-K_{\mathrm{s}}\right)_{0}<2.5 \mathrm{mag}$ but brighter for $\left(V-K_{\mathrm{s}}\right)_{0}>2.5 \mathrm{mag}$. This trend holds for distance modulus and colour excess in Brasseur et al. (2010), i.e. $\mu=15.50$ and $E(B-V)=0.034 \mathrm{mag}$, as well as for alternative data such as $E(B-V)=0.02$ and $\mu_{0}=15.50 \pm 0.20$ mag of Saviane et al. (1998). If we regard the metallicity claimed by Brasseur et al. (2010), $[\mathrm{Fe} / \mathrm{H}]=-1.40 \mathrm{dex}$, the absolute magnitude

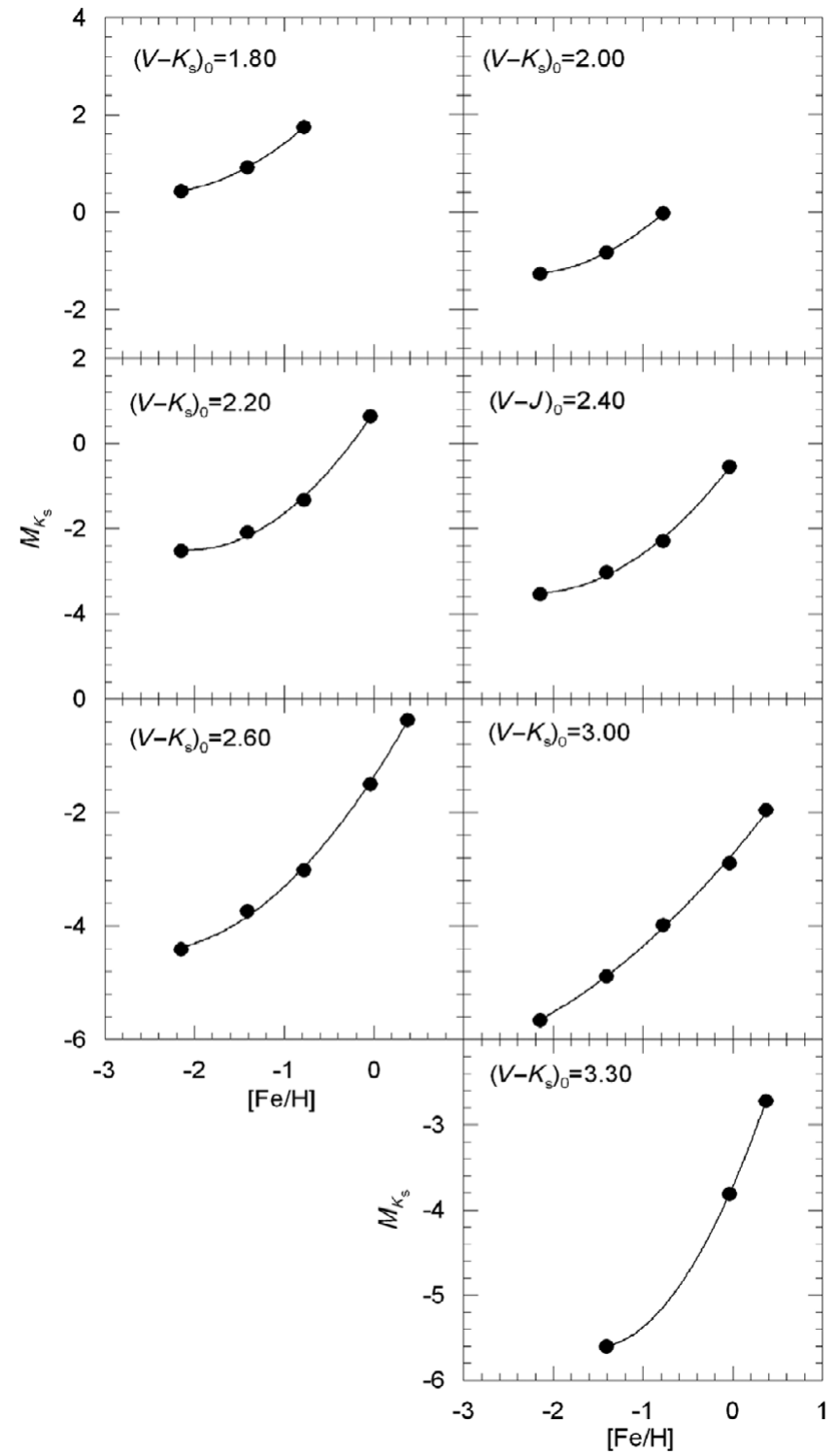

Figure 6. Calibration of the absolute magnitude $M_{K_{\mathrm{s}}}$ as a function of metallicity $[\mathrm{Fe} / \mathrm{H}]$ for seven colour indices.

colour diagram of the cluster NGC 1851 should coincide with that of M13 $([\mathrm{Fe} / \mathrm{H}]=-1.41 \mathrm{dex})$. The alternative metallicity of Rosenberg et al. (1999), $[\mathrm{Fe} / \mathrm{H}]=-1.03 \pm 0.06$ dex, should replace the absolute magnitude colour diagram of NGC 1851 between the diagrams of M13 and M71, which is not the case. $M_{K} \times\left(V-K_{\mathrm{s}}\right)_{0}$ diagrams for three clusters mentioned in this paragraph are not plotted because of space constraints.

Then, we transformed the $V, B-V$, and $V-I$ data of NGC 188 (Stetson et al. 2004) and M68 (Walker 1994) to the $K_{\mathrm{s}_{0}}$ magnitudes and $\left(V-K_{\mathrm{s}}\right)_{0}$ colours by Equation (3) and the procedure explained in Section 2.2. Colour excess $E(B-V)$, distance modulus $\mu_{0}$, and metallicity for the clusters are given in Table 14. The second reference for the cluster NGC 188 is given for the metallicity only, whereas the first 
Table 13. $M_{K_{\mathrm{s}}}$ Absolute Magnitudes Estimated for Five Galactic Clusters and the Numerical Values of $d_{i}(i=0,1,2)$ Coefficients in Equation (9)

\begin{tabular}{|c|c|c|c|c|c|c|c|c|c|c|c|c|c|c|c|c|c|c|c|c|c|}
\hline \multirow{2}{*}{$\begin{array}{l}\text { Cluster } \rightarrow \\
\left(V-K_{\mathrm{s}}\right)_{0}\end{array}$} & M92 & M13 & M71 & M67 & NGC 6791 & \multicolumn{6}{|r|}{ Cluster $\rightarrow$} & \multirow[t]{2}{*}{ M92 } & \multirow[t]{2}{*}{ M13 } & \multirow{2}{*}{$\begin{array}{l}\mathrm{M} 71 \\
M_{K_{\mathrm{s}}}\end{array}$} & \multirow[t]{2}{*}{ M67 } & \multirow[t]{2}{*}{ NGC 6791} & \multirow[b]{2}{*}{$d_{0}$} & \multirow[b]{2}{*}{$d_{1}$} & \multirow[b]{2}{*}{$d_{2}$} & \multirow[b]{2}{*}{$R^{2}$} & \multirow[b]{2}{*}[\mathrm{Fe}/\mathrm{H}]{$\mathrm{Int}}$. \\
\hline & & & $M_{K_{\mathrm{s}}}$ & & & $d_{0}$ & $d_{1}$ & $d_{2}$ & $R^{2}$ & {$[\mathrm{Fe} / \mathrm{H}]$ Int. } & $\left(V-K_{\mathrm{s}}\right)_{0}$ & & & & & & & & & & \\
\hline 1.75 & 0.957 & 1.456 & - & - & - & 2.4069 & 0.6743 & - & 1 & {$[-2.15,-1.41]$} & 2.78 & -5.072 & -4.293 & -3.502 & -2.187 & -1.224 & -2.0618 & 2.0624 & 0.3127 & 0.9993 & {$[-2.15,0.37]$} \\
\hline 1.76 & 0.849 & 1.345 & - & - & - & 2.2905 & 0.6705 & - & 1 & {$[-2.15,-1.41]$} & 2.79 & -5.105 & -4.322 & -3.527 & -2.222 & -1.263 & -2.0954 & 2.0480 & 0.3060 & 0.9993 & {$[-2.15,0.37]$} \\
\hline 1.77 & 0.742 & 1.236 & - & - & - & 2.1757 & 0.6667 & - & 1 & {$[-2.15,-1.41]$} & 2.80 & -5.138 & -4.350 & -3.550 & -2.255 & -1.302 & -2.1286 & 2.0342 & 0.2996 & 0.9993 & {$[-2.15,0.37]$} \\
\hline 1.78 & 0.638 & 1.128 & - & - & - & 2.0626 & 0.6627 & - & 1 & {$[-2.15,-1.41]$} & 2.81 & -5.170 & -4.378 & -3.574 & -2.289 & -1.340 & -2.1613 & 2.0209 & 0.2935 & 0.9993 & {$[-2.15,0.37]$} \\
\hline 1.79 & 0.535 & 1.023 & 1.845 & - & - & 3.3824 & 2.3393 & 0.4721 & 1 & {$[-2.15,-0.78]$} & 2.82 & -5.201 & -4.406 & -3.597 & -2.322 & -1.377 & -2.1937 & 2.0081 & 0.2876 & 0.9993 & {$[-2.15,0.37]$} \\
\hline 1.80 & 0.434 & 0.919 & 1.742 & - & - & 3.2861 & 2.3511 & 0.4766 & 1 & {$[-2.15,-0.78]$} & 2.83 & -5.232 & -4.434 & -3.621 & -2.355 & -1.414 & -2.2256 & 1.9957 & 0.2819 & 0.9993 & {$[-2.15,0.37]$} \\
\hline 1.81 & 0.335 & 0.816 & 1.641 & - & - & 3.1907 & 2.3618 & 0.4807 & 1 & {$[-2.15,-0.78]$} & 2.84 & -5.263 & -4.462 & -3.643 & -2.388 & -1.450 & -2.2572 & 1.9839 & 0.2766 & 0.9994 & {$[-2.15,0.37]$} \\
\hline 1.82 & 0.238 & 0.716 & 1.541 & - & - & 3.0961 & 2.3716 & 0.4847 & 1 & {$[-2.15,-0.78]$} & 2.85 & -5.292 & -4.490 & -3.666 & -2.421 & -1.486 & -2.2885 & 1.9725 & 0.2715 & 0.9994 & {$[-2.15,0.37]$} \\
\hline 1.83 & 0.142 & 0.617 & 1.443 & - & - & 3.0023 & 2.3804 & 0.4883 & 1 & {$[-2.15,-0.78]$} & 86 & -5.322 & -4.517 & -3.688 & -2.453 & -1.521 & -2.3194 & 1.9616 & 0.2666 & 0.9993 & {$[-2.15,0.37]$} \\
\hline 1.84 & 0.048 & 0.520 & 1.346 & - & - & 2.9094 & 2.3882 & 0.4917 & 1 & {$[-2.15,-0.78]$} & 87 & -5.350 & -4.545 & -3.711 & -2.485 & -1.555 & -2.3501 & 1.9512 & 0.2621 & 0.9993 & {$[-2.15,0.37]$} \\
\hline 1.85 & -0.045 & 0.424 & 1.250 & - & - & 2.8172 & 2.3951 & 0.4948 & 1 & {$[-2.15,-0.78]$} & 2. & -5.378 & -4.572 & -3.733 & -2.517 & -1.589 & -2.3804 & 1.9413 & 0.2578 & 0.9993 & {$[-2.15,0.37]$} \\
\hline 1.86 & -0.136 & 0.330 & 1.156 & - & - & 2.7259 & 2.4011 & 0.4976 & 1 & {$[-2.15,-0.78]$} & 2.89 & -5.406 & -4.599 & -3.754 & -2.549 & -1.623 & -2.4104 & 1.9318 & 0.2538 & 0.9993 & {$[-2.15,0.37]$} \\
\hline 1.87 & -0.225 & 0.237 & 1.063 & - & - & 2.6355 & 2.4061 & 0.5002 & 1 & {$[-2.15,-0.78]$} & 2.90 & -5.432 & -4.626 & -3.776 & -2.581 & -1.656 & -2.4402 & 1.9227 & 0.2501 & 0.9993 & {$[-2.15,0.37]$} \\
\hline 1.88 & -0.313 & 0.146 & 0.972 & - & - & 2.5458 & 2.4103 & 0.5025 & 1 & {$[-2.15,-0.78]$} & 2.91 & -5.459 & -4.653 & -3.797 & -2.612 & -1.688 & -2.4697 & 1.9142 & 0.2467 & 0.9992 & {$[-2.15,0.37]$} \\
\hline 1.89 & -0.400 & 0.057 & 0.881 & - & - & 2.4570 & 2.4136 & 0.5046 & 1 & {$[-2.15,-0.78]$} & 2.92 & -5.484 & -4.680 & -3.818 & -2.643 & -1.720 & -2.4989 & 1.9060 & 0.2436 & 0.9992 & {$[-2.15,0.37]$} \\
\hline 1.90 & -0.485 & -0.031 & 0.792 & - & - & 2.3689 & 2.4161 & 0.5064 & 1 & {$[-2.15,-0.78]$} & 2.93 & -5.509 & -4.706 & -3.839 & -2.675 & -1.751 & -2.5280 & 1.8983 & 0.2409 & 0.9991 & {$[-2.15,0.37]$} \\
\hline 1.91 & -0.569 & -0.118 & 0.705 & - & - & 2.2817 & 2.4177 & 0.5079 & 1 & {$[-2.15,-0.78]$} & 2.94 & -5.532 & -4.733 & -3.860 & -2.706 & -1.782 & -2.5568 & 1.8911 & 0.2384 & 0.9991 & {$[-2.15,0.37]$} \\
\hline 1.92 & -0.651 & -0.203 & 0.619 & - & - & 2.1952 & 2.4184 & 0.5091 & 1 & {$[-2.15,-0.78]$} & 2.95 & -5.556 & -4.759 & -3.881 & -2.736 & -1.813 & -2.5854 & 1.8842 & 0.2362 & 0.9990 & {$[-2.15,0.37]$} \\
\hline 1.93 & -0.732 & -0.286 & 0.534 & - & - & 2.1096 & 2.4184 & 0.5101 & 1 & {$[-2.15,-0.78]$} & 2.96 & -5.578 & -4.786 & -3.901 & -2.767 & -1.843 & -2.6138 & 1.8779 & 0.2344 & 0.9989 & {$[-2.15,0.37]$} \\
\hline 1.94 & -0.812 & -0.369 & 0.450 & - & - & 2.0247 & 2.4176 & 0.5108 & 1 & {$[-2.15,-0.78]$} & 2.97 & -5.600 & -4.812 & -3.921 & -2.798 & -1.873 & -2.6421 & 1.8719 & 0.2329 & 0.9989 & {$[-2.15,0.37]$} \\
\hline 1.95 & -0.890 & -0.449 & 0.367 & - & - & 1.9406 & 2.4160 & 0.5113 & 1 & {$[-2.15,-0.78]$} & 2.98 & -5.620 & -4.838 & -3.941 & -2.829 & -1.902 & -2.6707 & 1.8664 & 0.2317 & 0.9988 & {$[-2.15,0.37]$} \\
\hline 1.96 & -0.968 & -0.529 & 0.286 & - & - & 1.8573 & 2.4137 & 0.5115 & 1 & {$[-2.15,-0.78]$} & 2.99 & -5.640 & -4.864 & -3.961 & -2.859 & -1.931 & -2.6982 & 1.8613 & 0.2309 & 0.9986 & {$[-2.15,0.37]$} \\
\hline 1.97 & -1.044 & -0.607 & 0.206 & - & - & 1.7748 & 2.4106 & 0.5115 & 1 & {$[-2.15,-0.78]$} & 3.00 & -5.659 & -4.890 & -3.981 & -2.890 & -1.960 & -2.7260 & 1.8566 & 0.2304 & 0.9985 & {$[-2.15,0.37]$} \\
\hline 1.98 & -1.119 & -0.684 & 0.127 & - & - & 1.6931 & 2.4068 & 0.5112 & 1 & {$[-2.15,-0.78]$} & 3.01 & -5.677 & -4.916 & -4.001 & -2.920 & -1.988 & -2.7537 & 1.8524 & 0.2303 & 0.9984 & {$[-2.15,0.37]$} \\
\hline 1.99 & -1.192 & -0.760 & 0.049 & - & - & 1.6112 & 2.4023 & 0.5106 & 1 & {$[-2.15,-0.78]$} & 3.02 & -5.695 & -4.941 & -4.020 & -2.951 & -2.016 & -2.7813 & 1.8485 & 0.2303 & 0.9982 & {$[-2.15,0.37]$} \\
\hline 2.00 & -1.265 & -0.834 & -0.028 & - & - & 1.5320 & 2.3972 & 0.5098 & 1 & {$[-2.15,-0.78]$} & 3.03 & -5.711 & -4.967 & -4.040 & -2.981 & -2.044 & -2.8088 & 1.8451 & 0.2312 & 0.9980 & {$[-2.15,0.37]$} \\
\hline 2.01 & -1.337 & -0.908 & -0.103 & - & - & 1.4526 & 2.3913 & 0.5088 & 1 & {$[-2.15,-0.78]$} & 3.04 & -5.726 & -4.992 & -4.059 & -3.011 & -2.071 & -2.8362 & 1.8421 & 0.2322 & 0.9978 & {$[-2.15,0.37]$} \\
\hline 2.02 & -1.407 & -0.980 & -0.177 & - & - & 1.3740 & 2.3848 & 0.5075 & 1 & {$[-2.15,-0.78]$} & 3.05 & - & -5.017 & -4.078 & -3.042 & -2.098 & -2.8651 & 1.8452 & 0.2401 & 0.9957 & {$[-1.41,0.37]$} \\
\hline 2.03 & -1.477 & -1.050 & -0.251 & - & - & 1.2961 & 2.3777 & 0.5060 & 1 & {$[-2.15,-0.78]$} & 3.06 & - & -5.043 & -4.097 & -3.072 & -2.125 & -2.8910 & 1.8379 & 0.2359 & 0.9953 & {$[-1.41,0.37]$} \\
\hline 2.04 & -1.546 & -1.120 & -0.323 & - & - & 1.2191 & 2.3700 & 0.5042 & 1 & {$[-2.15,-0.78]$} & 3.07 & - & -5.068 & -4.116 & -3.103 & -2.152 & -2.9168 & 1.8307 & 0.2319 & 0.9949 & {$[-1.41,0.37]$} \\
\hline 2.05 & -1.613 & -1.189 & -0.394 & - & - & 1.1427 & 2.3617 & 0.5022 & 1 & {$[-2.15,-0.78]$} & 3.08 & - & -5.093 & -4.135 & -3.133 & -2.178 & -2.9426 & 1.8238 & 0.2281 & 0.9944 & {$[-1.41,0.37]$} \\
\hline 2.06 & -1.680 & -1.256 & -0.464 & - & - & 1.0672 & 2.3528 & 0.5000 & 1 & {$[-2.15,-0.78]$} & 3.09 & - & -5.117 & -4.154 & -3.163 & -2.204 & -2.9682 & 1.8171 & 0.2244 & 0.9939 & {$[-1.41,0.37]$} \\
\hline 2.07 & -1.746 & -1.322 & -0.533 & - & - & 0.9924 & 2.3433 & 0.4976 & 1 & {$[-2.15,-0.78]$} & 3.10 & - & -5.142 & -4.172 & -3.194 & -2.230 & -2.9938 & 1.8106 & 0.2210 & 0.9933 & {$[-1.41,0.37]$} \\
\hline 2.08 & -1.811 & -1.388 & -0.601 & - & - & 0.9184 & 2.3333 & 0.4949 & 1 & {$[-2.15,-0.78]$} & 3.11 & - & -5.167 & - & -3.224 & -2.256 & -3.1378 & 2.1872 & 0.5307 & 1 & {$[-1.41,0.37]$} \\
\hline 2.09 & -1.875 & -1.452 & -0.667 & - & - & 0.8451 & 2.3228 & 0.4920 & 1 & {$[-2.15,-0.78]$} & 3.12 & - & -5.191 & - & -3.255 & -2.281 & -3.1680 & 2.1964 & 0.5401 & 1 & {$[-1.41,0.37]$} \\
\hline 2.10 & -1.938 & -1.515 & -0.733 & - & - & 0.7726 & 2.3118 & 0.4889 & 1 & {$[-2.15,-0.78]$} & 3.13 & - & -5.215 & - & -3.286 & -2.307 & -3.1982 & 2.2061 & 0.5500 & 1 & {$[-1.41,0.37]$} \\
\hline 2.11 & -2.000 & -1.577 & -0.798 & - & - & 0.7008 & 2.3003 & 0.4856 & 1 & {$[-2.15,-0.78]$} & 3.14 & - & -5.240 & - & -3.316 & -2.332 & -3.2285 & 2.2162 & 0.5602 & 1 & {$[-1.41,0.37]$} \\
\hline 2.12 & -2.062 & -1.638 & -0.862 & - & - & 0.6298 & 2.2884 & 0.4821 & 1 & {$[-2.15,-0.78]$} & 3.15 & - & -5.264 & - & -3.347 & -2.357 & -3.2588 & 2.2266 & 0.5708 & 1 & {$[-1.41,0.37]$} \\
\hline 2.13 & -2.123 & -1.699 & -0.925 & - & - & 0.5595 & 2.2760 & 0.4784 & 1 & {$[-2.15,-0.78]$} & 3.16 & - & -5.287 & - & -3.378 & -2.382 & -3.2891 & 2.2375 & 0.5818 & 1 & {$[-1.41,0.37]$} \\
\hline 2.14 & -2.183 & -1.758 & -0.987 & - & - & 0.4900 & 2.2632 & 0.4744 & 1 & {$[-2.15,-0.78]$} & 3.17 & - & -5.311 & - & -3.408 & -2.406 & -3.3195 & 2.2488 & 0.5931 & 1 & {$[-1.41,0.37]$} \\
\hline 2.15 & -2.242 & -1.816 & -1.048 & 0.960 & - & 1.0614 & 3.202 & 0.7802 & 0.9973 & {$[-2.15,-0.04]$} & 3.18 & - & -5.335 & - & -3.439 & -2.431 & -3.3499 & 2.2605 & 0.6048 & 1 & {$[-1.41,0.37]$} \\
\hline 2.16 & -2.301 & -1.874 & -1.108 & 0.895 & - & 0.9953 & 3.1913 & 0.7768 & 0.9973 & {$[-2.15,-0.04]$} & 3.19 & - & -5.358 & - & -3.470 & -2.455 & -3.3804 & 2.2725 & 0.6169 & 1 & {$[-1.41,0.37]$} \\
\hline
\end{tabular}


Table 13. Continued.

\begin{tabular}{|c|c|c|c|c|c|c|c|c|c|c|c|c|c|c|c|c|c|c|c|c|c|}
\hline \multirow{2}{*}{$\begin{array}{l}\text { Cluster } \rightarrow \\
\left(V-K_{\mathrm{s}}\right)_{0}\end{array}$} & M92 & M13 & M71 & M67 & NGC 6791 & \multicolumn{6}{|r|}{ Cluster $\rightarrow$} & M92 & M13 & M71 & M67 & NGC 6791 & & & & & \\
\hline & & & $M_{K_{\mathrm{S}}}$ & & & $d_{0}$ & $d_{1}$ & $d_{2}$ & $R^{2}$ & {$[\mathrm{Fe} / \mathrm{H}] \mathrm{Int}$.} & $\left(V-K_{\mathrm{s}}\right)_{0}$ & \multicolumn{5}{|c|}{$M_{K_{\mathrm{s}}}$} & $d_{0}$ & $d_{1}$ & $d_{2}$ & $R^{2}$ & {$[\mathrm{Fe} / \mathrm{H}]$ Int. } \\
\hline 2.17 & -2.359 & -1.930 & -1.167 & 0.829 & - & 0.9295 & 3.1795 & 0.7731 & 0.9973 & {$[-2.15,-0.04]$} & 3.20 & - & -5.381 & - & -3.501 & -2.479 & -3.4110 & 2.2848 & 0.6293 & 1 & {$[-1.41,0.37]$} \\
\hline 2.18 & -2.416 & -1.986 & -1.225 & 0.764 & - & 0.8640 & 3.1667 & 0.7689 & 0.9973 & {$[-2.15,-0.04]$} & 3.21 & - & -5.405 & - & -3.532 & -2.504 & -3.4415 & 2.2975 & 0.6421 & 1 & {$[-1.41,0.37]$} \\
\hline 2.19 & -2.473 & -2.041 & -1.282 & 0.700 & - & 0.7987 & 3.1528 & 0.7643 & 0.9972 & {$[-2.15,-0.04]$} & 22 & - & -5.427 & - & -3.564 & -2.528 & -3.4722 & 2.3105 & 0.6551 & 1 & {$[-1.41,0.37]$} \\
\hline 2.20 & -2.529 & -2.094 & -1.339 & 0.635 & - & 0.7338 & 3.1381 & 0.7594 & 0.9972 & {$[-2.15,-0.04]$} & 23 & - & -5.450 & - & -3.595 & -2.552 & -3.5029 & 2.3237 & 0.6685 & 1 & {$[-1.41,0.37]$} \\
\hline .21 & -2.584 & -2.148 & -1.394 & 0.572 & - & 0.6693 & 3.1225 & 0.7541 & 0.9972 & {$[-2.15,-0.04]$} & & - & -5.473 & - & -3.626 & -2.575 & -3.5337 & 2.3372 & 0.6822 & 1 & {$[-1.41,0.37]$} \\
\hline .22 & -2.639 & -2.200 & -1.449 & 0.508 & - & 0.6052 & 3.1062 & 0.7485 & 0.9972 & {$[-2.15,-0.04]$} & 25 & - & -5.495 & - & -3.657 & -2.599 & -3.5645 & 2.3510 & 0.6962 & 1 & {$[-1.41,0.37]$} \\
\hline .23 & -2.694 & -2.251 & -1.503 & 0.445 & - & 0.5414 & 3.0890 & 0.7425 & 0.9972 & {$[-2.15,-0.04]$} & & - & -5.517 & - & -3.6 & -2 & -3.5953 & 2.3650 & 0.7105 & 1 & {$[-1.41,0.37]$} \\
\hline 2.24 & -2.747 & -2.302 & -1.556 & 0.382 & - & 0.4781 & 3.0712 & 0.7363 & 0.9972 & {$[-2.15,-0.04]$} & & - & -5.539 & - & -3.720 & -2.647 & -3.6262 & 2.3792 & 0.7250 & 1 & {$[-1.41,0.37]$} \\
\hline .25 & -2.800 & -2.352 & -1.608 & 0.320 & - & 0.4152 & 3.0527 & 0.7298 & 0.9972 & {$[-2.15,-0.04]$} & & - & -5.561 & - & -3.752 & -2.670 & -3.6572 & 2.3935 & 0.7398 & 1 & {$[-1.41,0.37]$} \\
\hline .26 & -2.853 & -2.401 & -1.660 & 0.258 & - & 0.3527 & 3.0337 & 0.7230 & 0.9972 & {$[-2.15,-0.04]$} & 3.29 & - & -5.583 & - & -3.783 & -2.694 & -3.6881 & 2.4080 & 0.7548 & 1 & {$[-1.41,0.37]$} \\
\hline .27 & -2.905 & -2.450 & -1.710 & 0.197 & - & 0.2908 & 3.0140 & 0.7160 & 0.9972 & {$[-2.15,-0.04]$} & 3.30 & - & -5.604 & - & -3.815 & -2.717 & -3.7192 & 2.4227 & 0.7700 & 1 & {$[-1.41,0.37]$} \\
\hline .28 & -2.957 & -2.498 & -1.760 & 0.136 & - & 0.2293 & 2.9939 & 0.7087 & 0.9972 & {$[-2.15,-0.04]$} & 3.31 & - & -5.625 & - & -3.846 & -2.741 & -3.7502 & 2.4374 & 0.7854 & 1 & {$[-1.41,0.37]$} \\
\hline 29 & -3.008 & -2.545 & -1.809 & 0.076 & - & 0.1683 & 2.9732 & 0.7012 & 0.9972 & {$[-2.15,-0.04]$} & 3.32 & - & -5.646 & - & -3.878 & -2.764 & -3.7812 & 2.4522 & 0.8010 & 1 & {$[-1.41,0.37]$} \\
\hline 2.30 & -3.059 & -2.591 & -1.857 & 0.016 & - & 0.1079 & 2.9522 & 0.6935 & 0.9972 & {$[-2.15,-0.04]$} & 3.33 & - & -5.667 & - & -3.910 & -2.788 & -3.8123 & 2.4671 & 0.8168 & 1 & {$[-1.41,0.37]$} \\
\hline 2.31 & -3.109 & -2.637 & -1.905 & -0.043 & - & 0.0480 & 2.9307 & 0.6856 & 0.9972 & {$[-2.15,-0.04]$} & 3.34 & - & -5.688 & - & -3.941 & -2.811 & -3.8433 & 2.4820 & 0.8327 & 1 & {$[-1.41,0.37]$} \\
\hline 2.32 & -3.159 & -2.683 & -1.952 & -0.102 & - & -0.1140 & 2.9089 & 0.6774 & 0.9972 & {$[-2.15,-0.04]$} & 3.35 & - & -5.708 & - & -3.973 & -2.834 & -3.8744 & 2.4969 & 0.8487 & 1 & {$[-1.41,0.37]$} \\
\hline 2.33 & -3.209 & -2.727 & -1.998 & -0.160 & - & -0.0702 & 2.8868 & 0.6692 & 0.9972 & {$[-2.15,-0.04]$} & 3.36 & - & -5.728 & - & -4.004 & -2.858 & -3.9054 & 2.5117 & 0.8647 & 1 & {$[-1.41,0.37]$} \\
\hline 2.34 & -3.257 & -2.771 & -2.043 & -0.217 & - & -0.1285 & 2.8644 & 0.6607 & 0.9972 & {$[-2.15,-0.04]$} & 3.37 & - & -5.747 & - & -4.036 & -2.881 & -3.9364 & 2.5264 & 0.8809 & 1 & {$[-1.41,0.37]$} \\
\hline 2.35 & -3.306 & -2.814 & -2.088 & -0.274 & - & -0.1862 & 2.8418 & 0.6521 & 0.9972 & {$[-2.15,-0.04]$} & 3.38 & - & -5.767 & - & -4.068 & -2.904 & -3.9673 & 2.5411 & 0.8971 & 1 & {$[-1.41,0.37]$} \\
\hline 2.36 & -3.354 & -2.857 & -2.132 & -0.331 & - & -0.2433 & 2.8189 & 0.6434 & 0.9972 & {$[-2.15,-0.04]$} & 3.39 & - & -5.786 & - & -4.099 & -2.928 & -3.9982 & 2.5556 & 0.9133 & 1 & {$[-1.41,0.37]$} \\
\hline 2.37 & -3.402 & -2.900 & -2.176 & -0.386 & - & -0.2998 & 2.7959 & 0.6345 & 0.9973 & {$[-2.15,-0.04]$} & 3.40 & - & -5.805 & - & -4.130 & -2.951 & -4.0290 & 2.5699 & 0.9294 & 1 & {$[-1.41,0.37]$} \\
\hline 2.38 & -3.450 & -2.941 & -2.218 & -0.442 & - & -0.3558 & 2.7727 & 0.6255 & 0.9973 & {$[-2.15,-0.04]$} & 3.41 & - & -5.823 & - & -4.162 & -2.974 & -4.0597 & 2.5839 & 0.9455 & 1 & {$[-1.41,0.37]$} \\
\hline 2.39 & -3.497 & -2.982 & -2.260 & -0.496 & - & -0.4111 & 2.7494 & 0.6164 & 0.9973 & {$[-2.15,-0.04]$} & 3.42 & - & -5.841 & - & -4.193 & -2.997 & -4.0903 & 2.5977 & 0.9616 & 1 & {$[-1.41,0.37]$} \\
\hline 2.40 & -3.543 & -3.023 & -2.302 & -0.550 & - & -0.4658 & 2.7259 & 0.6072 & 0.9973 & {$[-2.15,-0.04]$} & 3.43 & - & -5.859 & - & -4.224 & -3.021 & -4.1208 & 2.6112 & 0.9775 & 1 & {$[-1.41,0.37]$} \\
\hline 2.41 & -3.590 & -3.063 & -2.343 & -0.604 & - & -0.5200 & 2.7024 & 0.5980 & 0.9974 & {$[-2.15,-0.04]$} & 3.44 & - & -5.877 & - & -4.254 & -3.044 & -4.1511 & 2.6244 & 0.9932 & 1 & {$[-1.41,0.37]$} \\
\hline 2.42 & -3.636 & -3.103 & -2.383 & -0.656 & - & -0.5735 & 2.6789 & 0.5886 & 0.9974 & {$[-2.15,-0.04]$} & & - & -5.894 & - & -4.285 & 67 & -4.1813 & 2.6372 & 1.0088 & 1 & {$[-1.41,0.37]$} \\
\hline 2.43 & -3.681 & -3.142 & -2.422 & -0.709 & - & -0.6264 & 2.6554 & 0.5792 & 0.9974 & {$[-2.15,-0.04]$} & 3.46 & - & -5.911 & - & -4.316 & -3.091 & -4.2112 & 2.6495 & 1.0241 & 1 & {$[-1.41,0.37]$} \\
\hline 2.44 & -3.727 & -3.181 & -2.461 & -0.760 & - & -0.6787 & 2.6318 & 0.5697 & 0.9975 & {$[-2.15,-0.04]$} & 3.47 & - & -5.927 & - & -4.346 & -3.114 & -4.2410 & 2.6613 & 1.0392 & 1 & {$[-1.41,0.37]$} \\
\hline 2.45 & -3.771 & -3.219 & -2.500 & -0.811 & 0.619 & -0.6056 & 2.8777 & 0.6641 & 0.9974 & {$[-2.15,0.37]$} & 3.48 & - & -5.944 & - & -4.376 & -3.137 & -4.2706 & 2.6726 & 1.0540 & 1 & {$[-1.41,0.37]$} \\
\hline 2.46 & -3.816 & -3.257 & -2.538 & -0.862 & 0.544 & -0.6626 & 2.8415 & 0.6495 & 0.9976 & {$[-2.15,0.37]$} & 3.49 & - & -5.959 & - & -4.405 & -3.161 & -4.2998 & 2.6833 & 1.0684 & 1 & {$[-1.41,0.37]$} \\
\hline 2.47 & -3.860 & -3.294 & -2.575 & -0.911 & 0.470 & -0.7186 & 2.8060 & 0.6353 & 0.9977 & {$[-2.15,0.37]$} & 3.50 & - & -5.975 & - & -4.435 & -3.184 & -4.3288 & 2.6934 & 1.0824 & 1 & {$[-1.41,0.37]$} \\
\hline 2.48 & -3.904 & -3.331 & -2.611 & -0.961 & 0.397 & -0.7736 & 2.7714 & 0.6213 & 0.9978 & {$[-2.15,0.37]$} & 3. & - & -5.990 & - & -4.464 & -3.207 & -4.3575 & 2.7028 & 1.0960 & 1 & {$[-1.41,0.37]$} \\
\hline 2.49 & -3.948 & -3.367 & -2.648 & -1.009 & 0.326 & -0.8278 & 2.7377 & 0.6076 & 0.9979 & {$[-2.15,0.37]$} & 3.52 & - & -6.004 & - & -4.493 & -3.231 & -4.3859 & 2.7114 & 1.1091 & 1 & {$[-1.41,0.37]$} \\
\hline 2.50 & -3.992 & -3.403 & -2.683 & -1.057 & 0.256 & -0.8810 & 2.7047 & 0.5941 & 0.9980 & {$[-2.15,0.37]$} & 3.53 & - & -6.018 & - & -4.521 & -3.254 & -4.4139 & 2.7192 & 1.1216 & 1 & {$[-1.41,0.37]$} \\
\hline 2.51 & -4.035 & -3.439 & -2.718 & -1.104 & 0.188 & -0.9332 & 2.6725 & 0.5809 & 0.9981 & {$[-2.15,0.37]$} & 3.54 & - & -6.032 & - & -4.549 & -3.278 & -4.4415 & 2.7262 & 1.1336 & 1 & {$[-1.41,0.37]$} \\
\hline 2.52 & -4.077 & -3.475 & -2.753 & -1.151 & 0.121 & -0.9846 & 2.6411 & 0.5679 & 0.9982 & {$[-2.15,0.37]$} & 3.55 & - & -6.045 & - & -4.576 & -3.301 & -4.4687 & 2.7322 & 1.1449 & 1 & {$[-1.41,0.37]$} \\
\hline
\end{tabular}


Table 13. Continued.

\begin{tabular}{|c|c|c|c|c|c|c|c|c|c|c|c|c|c|c|c|c|c|c|c|c|c|}
\hline \multirow{2}{*}{$\begin{array}{l}\text { Cluster } \rightarrow \\
\left(V-K_{\mathrm{s}}\right)_{0} \\
\end{array}$} & \multirow[t]{2}{*}{ M92 } & \multirow[t]{2}{*}{ M13 } & \multirow{2}{*}{$\begin{array}{l}\mathrm{M} 71 \\
M_{K_{\mathrm{s}}}\end{array}$} & \multirow[t]{2}{*}{ M67 } & \multirow[t]{2}{*}{ NGC 6791} & \multirow[b]{2}{*}{$d_{0}$} & \multirow[b]{2}{*}{$d_{1}$} & \multirow[b]{2}{*}{$d_{2}$} & \multirow[b]{2}{*}{$R^{2}$} & \multirow[b]{2}{*}{ [Fe/H] Int. } & \multirow{2}{*}{$\begin{array}{l}\text { Cluster } \rightarrow \\
\left(V-K_{\mathrm{s}}\right)_{0}\end{array}$} & \multirow[t]{2}{*}{ M92 } & \multirow[t]{2}{*}{ M13 } & \multirow{2}{*}{$\begin{array}{r}\mathrm{M} 71 \\
M_{K_{\mathrm{s}}}\end{array}$} & \multirow[t]{2}{*}{ M67 } & \multirow[t]{2}{*}{ NGC 6791} & \multirow[b]{2}{*}{$d_{0}$} & \multirow[b]{2}{*}{$d_{1}$} & \multirow[b]{2}{*}{$d_{2}$} & \multirow[b]{2}{*}{$R^{2}$} & \multirow[b]{2}{*}[\mathrm{Fe}/\mathrm{H}]{ Int. } \\
\hline & & & & & & & & & & & & & & & & & & & & & \\
\hline 2.53 & -4.120 & -3.510 & -2.787 & -1.198 & 0.055 & -1.0351 & 2.6105 & 0.5552 & 0.9982 & {$[-2.15,0.37]$} & 3.56 & - & -6.058 & - & -4.603 & -3.324 & -4.4954 & 2.7373 & 1.1555 & 1 & {$[-1.41,0.37]$} \\
\hline 2.54 & -4.162 & -3.544 & -2.821 & -1.243 & -0.009 & -1.0848 & 2.5806 & 0.5427 & 0.9983 & {$[-2.15,0.37]$} & 3.57 & - & -6.070 & - & -4.629 & -3.348 & -4.5217 & 2.7412 & 1.1654 & 1 & {$[-1.41,0.37]$} \\
\hline 2.55 & -4.204 & -3.578 & -2.854 & -1.288 & -0.072 & -1.1336 & 2.5515 & 0.5303 & 0.9984 & {$[-2.15,0.37]$} & 58 & - & -6.082 & - & -4.655 & -3.371 & -4.5474 & 2.7441 & 1.1745 & 1 & {$[-1.41,0.37]$} \\
\hline 2.56 & -4.245 & -3.612 & -2.886 & -1.333 & -0.134 & -1.1816 & 2.5231 & 0.5184 & 0.9984 & {$[-2.15,0.37]$} & 59 & - & -6.093 & - & -4.680 & -3.395 & -4.5725 & 2.7458 & 1.1826 & 1 & {$[-1.41,0.37]$} \\
\hline 2.57 & -4.287 & -3.646 & -2.919 & -1.377 & -0.195 & -1.2287 & 2.4954 & 0.5067 & 0.9985 & {$[-2.15,0.37]$} & 60 & - & -6.104 & - & -4.705 & -3.418 & -4.5971 & 2.7462 & 1.1899 & 1 & {$[-1.41,0.37]$} \\
\hline 2.58 & -4.327 & -3.679 & -2.950 & -1.420 & -0.2 & -1.2751 & 2.4684 & 0.4951 & 0.9986 & {$[-2.15,0.3$} & 3.61 & - & -6.114 & - & -4.729 & -3.441 & -4.62 & 2.7452 & 1.1961 & 1 & {$[-1.41,0.37]$} \\
\hline 2.59 & -4.368 & -3.712 & -2.981 & -1.463 & -0.3 & 07 & 2.4422 & 0.4838 & 0.9986 & {$[-2.15,0$} & 3.62 & - & -6.123 & - & -4.752 & -3. & -4.6 & 2.7429 & 1.2013 & 1 & {$[-1.41,0.37]$} \\
\hline 2.60 & -4.408 & -3.745 & -3.012 & -1.505 & 0.36 & -1.3656 & 2.4166 & 0.4728 & 0.9987 & {$[-2.15,0.37]$} & 3.63 & - & -6.132 & - & -4.774 & -3.4 & -4.6667 & 2.7391 & 1.2053 & 1 & {$[-1.41,0.37]$} \\
\hline 2.61 & -4.448 & -3.778 & -3.043 & -1.547 & -0.425 & -1.4096 & 2.3917 & 0.4619 & 0.9987 & {$[-2.15,0.37]$} & & - & -6.141 & - & -4.796 & -3.512 & -4.6885 & 2.7337 & 1.2082 & 1 & {$[-1.41,0.37]$} \\
\hline 2.62 & -4.488 & -3.810 & -3.073 & -1.588 & -0.479 & -1.4530 & 2.3675 & 0.4513 & 0.9988 & {$[-2.15,0.37]$} & 65 & - & -6.149 & - & -4.817 & -3.535 & -4.7094 & 2.7266 & 1.2097 & 1 & {$[-1.41,0.37]$} \\
\hline 2.63 & -4.527 & -3.842 & -3.102 & -1.629 & -0.533 & -1.4957 & 2.3439 & 0.4409 & 0.9988 & {$[-2.15,0.37]$} & 3 & - & -6.156 & - & -4.836 & -3.558 & -4.7295 & 2.7178 & 1.2099 & 1 & {$[-1.41,0.37]$} \\
\hline 2.64 & -4.566 & -3.873 & -3.131 & -1.669 & -0.586 & -1.5376 & 2.3209 & 0.4307 & 0.9988 & {$[-2.15,0.37]$} & 3 & - & -6.163 & - & -4.855 & -3.5 & -4.7486 & 2.7072 & 1.2087 & 1 & {$[-1.41,0.37]$} \\
\hline 2.65 & -4.605 & -3.905 & -3.160 & -1.709 & -0.637 & -1.5789 & 2.2986 & 0.4208 & 0.9989 & {$[-2.15,0.37]$} & & - & -6.169 & - & -4.873 & -3.6 & -4.7669 & 2.6947 & 1.2060 & 1 & {$[-1.41,0.37]$} \\
\hline 2.66 & -4.643 & -3.936 & -3.189 & -1.748 & -0.688 & -1.6195 & 2.2770 & 0.4111 & 0.9989 & {$[-2.15,0.37]$} & 3.69 & - & -6.174 & - & -4.889 & -3.628 & -4.7841 & 2.6802 & 1.2016 & 1 & {$[-1.41,0.37]$} \\
\hline 2.67 & -4.681 & -3.967 & -3.217 & -1.787 & -0.737 & -1.6595 & 2.2559 & 0.4016 & 0.9990 & {$[-2.15,0.37]$} & 3.70 & - & - & - & -4.905 & -3.651 & -4.7826 & 3.0581 & - & 1 & {$[-0.04,0.37]$} \\
\hline 2.68 & -4.719 & -3.997 & -3.244 & -1.826 & -0.786 & -1.6989 & 2.2354 & 0.3924 & 0.9990 & {$[-2.15,0.37]$} & 3.71 & - & - & - & -4.919 & -3.674 & -4.7978 & 3.0367 & - & 1 & {$[-0.04,0.37]$} \\
\hline 2.69 & -4.756 & -4.028 & -3.272 & -1.864 & -0.833 & -1.7377 & 2.2156 & 0.3834 & 0.9990 & {$[-2.15,0.37]$} & 3.72 & - & - & - & -4.932 & -3.697 & -4.8118 & 3.0125 & - & 1 & {$[-0.04,0.37]$} \\
\hline 2.70 & -4.793 & -4.058 & -3.299 & -1.901 & -0.880 & -1.7758 & 2.1963 & 0.3746 & 0.9991 & {$[-2.15,0.37]$} & 3.73 & - & - & - & -4.944 & -3.720 & -4.8247 & 2.9852 & - & 1 & {$[-0.04,0.37]$} \\
\hline 2.71 & -4.829 & -4.088 & -3.325 & -1.938 & -0.926 & -1.8134 & 2.1776 & 0.3660 & 0.9991 & {$[-2.15,0.37]$} & 3.74 & - & - & - & -4.954 & -3.743 & -4.8363 & 2.9547 & - & 1 & {$[-0.04,0.37]$} \\
\hline 2.72 & -4.865 & -4.118 & -3.351 & -1.975 & -0.971 & -1.8504 & 2.1595 & 0.3577 & 0.9991 & {$[-2.15,0.37]$} & 75 & - & - & - & -4.963 & -3.766 & -4.8466 & 2.9209 & - & 1 & {$[-0.04,0.37]$} \\
\hline 2.73 & -4.901 & -4.148 & -3.377 & -2.011 & -1.015 & -1.8869 & 2.1420 & 0.3496 & 0.9992 & {$[-2.15,0.37]$} & 3.76 & - & - & - & -4.971 & -3.789 & -4.8555 & 2.8837 & - & 1 & {$[-0.04,0.37]$} \\
\hline 2.74 & -4.936 & -4.177 & -3.403 & -2.047 & -1.058 & -1.9229 & 2.1250 & 0.3417 & 0.9992 & {$[-2.15,0.37]$} & 3.77 & - & - & - & -4.977 & -3.811 & -4.8631 & 2.8429 & - & 1 & {$[-0.04,0.37]$} \\
\hline 2.75 & -4.971 & -4.206 & -3.428 & -2.083 & -1.101 & 1.9583 & 2.1085 & 0.3341 & 0.9992 & {$[-2.15,0.37]$} & & - & - & - & -4.981 & -3.834 & -4.8691 & 2.7984 & - & 1 & {$[-0.04,0.37]$} \\
\hline 2.76 & -5.005 & -4.235 & -3.453 & -2.118 & -1.142 & -1.9933 & 2.0926 & 0.3267 & 0.9992 & {$[-2.15,0.37]$} & 3.79 & - & - & - & -4.984 & -3.856 & -4.8736 & 2.7500 & - & 1 & {$[-0.04,0.37]$} \\
\hline 2.77 & -5.039 & -4.264 & -3.478 & -2.153 & -1.183 & -2.0278 & 2.0772 & 0.3196 & 0.9993 & {$[-2.15,0.37]$} & 3.80 & - & - & - & -4.984 & -3.878 & -4.8765 & 2.6975 & - & 1 & {$[-0.04,0.37]$} \\
\hline
\end{tabular}

Note. The 11th and 22nd columns give the range of the metallicity $[\mathrm{Fe} / \mathrm{H}](\mathrm{dex})$ for the star whose absolute magnitude would be estimated. $R^{2}$ is the square of the correlation coefficient. 


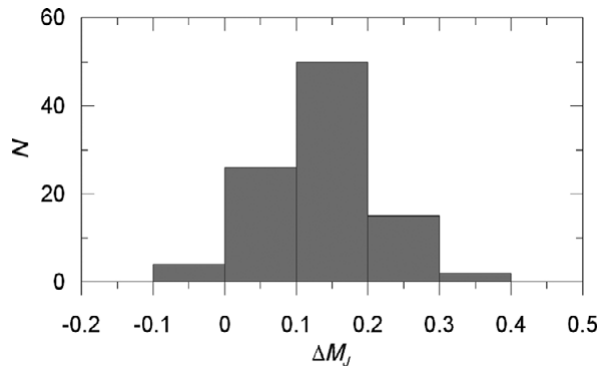

Figure 7. Histogram of the residuals for $\Delta M_{J}$.

Table 14. Data for the Clusters Used for the Application of the Procedure

\begin{tabular}{lcccc}
\hline \hline Cluster & $\begin{array}{c}E(B-V) \\
(\mathrm{mag})\end{array}$ & $\begin{array}{c}\mu_{0} \\
(\mathrm{mag})\end{array}$ & $\begin{array}{c}{[\mathrm{Fe} / \mathrm{H}]} \\
(\mathrm{dex})\end{array}$ & Ref. \\
\hline M5 & 0.038 & 14.330 & -1.17 & 1,2 \\
M68 & 0.060 & 14.994 & -2.01 & 3 \\
NGC 188 & 0.087 & 11.130 & -0.01 & 4,5 \\
\hline \hline
\end{tabular}

References: (1) Brasseur et al. (2010); (2) Sandquist et al. (1996); (3) VandenBerg \& Clem (2003); (4) Stetson, McClure, \& VandenBerg (2004); (5) Meibom et al. (2009).

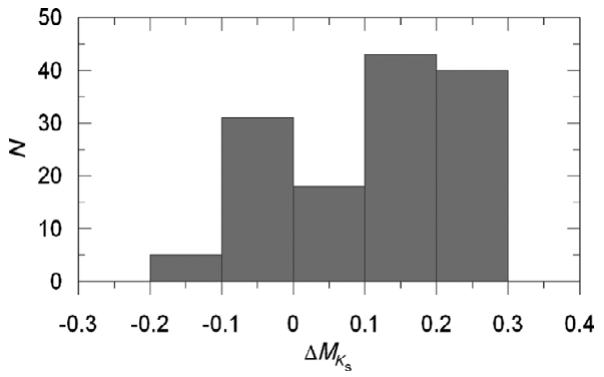

Figure 8. Histogram of the residuals for $\Delta M_{K_{\mathrm{s}}}$.

reference refers to the colour excess and the distance modulus. The $K_{\mathrm{s}_{0}}$ magnitudes and $\left(V-K_{\mathrm{s}}\right)_{0}$ colours as well as the original $V, B-V$, and $V-I$ data are given in Table 18 .

We evaluated the $M_{K_{\mathrm{s}}}$ absolute magnitude by Equation (9) for a set of $\left(V-K_{\mathrm{s}}\right)_{0}$ colour indices where the clusters are defined. The results are given in Table 19. The columns refer to (1) $\left(V-K_{\mathrm{s}}\right)_{0}$ colour index; (2) $\left(M_{K_{\mathrm{s}}}\right)_{\mathrm{cl}}$, the absolute magnitude for a cluster estimated by its colour-magnitude diagram; (3) $\left(M_{K_{\mathrm{s}}}\right)_{\mathrm{ev}}$, the absolute magnitude estimated by the procedure; (4) $\Delta M_{K_{s}}$, absolute magnitude residuals. Also, the metallicity for each cluster is indicated near the name of the cluster. The differences between the absolute magnitudes estimated by the procedure presented in this study and those evaluated via the colour-magnitude diagrams of the clusters (the residuals) lie in a short interval, i.e. -0.10 and +0.27 mag. The mean and the standard deviation of the residuals are $\left\langle\Delta M_{K_{\mathrm{s}}}\right\rangle=0.109$ and $\sigma_{M_{K_{\mathrm{s}}}}=0.123 \mathrm{mag}$, respectively. The distribution of the residuals is given in Table 20 and Figure 8. The residuals for the cluster NGC
Table 15. $J_{0} \times(V-J)_{0}$ Fiducial Giant Sequences for the Clusters M5 and M68 Used in the Application of the Procedure

\begin{tabular}{|c|c|c|c|c|c|}
\hline Cluster & $V$ & $V-J$ & $J$ & $(V-J)_{0}$ & $J_{0}$ \\
\hline \multirow[t]{24}{*}{ M5 } & 12.31 & 2.59 & 9.72 & 2.505 & 9.687 \\
\hline & 12.50 & 2.47 & 10.03 & 2.385 & 9.997 \\
\hline & 12.68 & 2.36 & 10.32 & 2.275 & 10.287 \\
\hline & 12.86 & 2.27 & 10.59 & 2.185 & 10.557 \\
\hline & 13.09 & 2.18 & 10.91 & 2.095 & 10.877 \\
\hline & 13.29 & 2.12 & 11.17 & 2.035 & 11.137 \\
\hline & 13.49 & 2.06 & 11.43 & 1.975 & 11.397 \\
\hline & 13.71 & 2.00 & 11.71 & 1.915 & 11.677 \\
\hline & 13.91 & 1.95 & 11.96 & 1.865 & 11.927 \\
\hline & 14.14 & 1.89 & 12.25 & 1.805 & 12.217 \\
\hline & 14.35 & 1.84 & 12.51 & 1.755 & 12.477 \\
\hline & 14.57 & 1.79 & 12.78 & 1.705 & 12.747 \\
\hline & 14.80 & 1.74 & 13.06 & 1.655 & 13.027 \\
\hline & 15.04 & 1.70 & 13.34 & 1.615 & 13.307 \\
\hline & 15.27 & 1.67 & 13.60 & 1.585 & 13.567 \\
\hline & 15.55 & 1.63 & 13.92 & 1.545 & 13.887 \\
\hline & 15.81 & 1.60 & 14.21 & 1.515 & 14.177 \\
\hline & 16.05 & 1.57 & 14.48 & 1.485 & 14.447 \\
\hline & 16.33 & 1.54 & 14.79 & 1.455 & 14.757 \\
\hline & 16.57 & 1.51 & 15.06 & 1.425 & 15.027 \\
\hline & 16.83 & 1.50 & 15.33 & 1.415 & 15.297 \\
\hline & 17.08 & 1.48 & 15.60 & 1.395 & 15.567 \\
\hline & 17.32 & 1.47 & 15.85 & 1.385 & 15.817 \\
\hline & 17.54 & 1.46 & 16.08 & 1.375 & 16.047 \\
\hline \multirow[t]{11}{*}{ M68 } & 1.234 & 1.196 & 12.497 & 2.203 & 10.294 \\
\hline & 1.202 & 1.154 & 12.844 & 2.146 & 10.698 \\
\hline & 1.047 & 0.951 & 13.498 & 1.868 & 11.630 \\
\hline & 0.965 & 0.855 & 14.143 & 1.733 & 12.410 \\
\hline & 0.905 & 0.775 & 14.744 & 1.624 & 13.120 \\
\hline & 0.863 & 0.732 & 15.209 & 1.561 & 13.648 \\
\hline & 0.803 & 0.669 & 15.775 & 1.471 & 14.304 \\
\hline & 0.792 & 0.646 & 16.228 & 1.442 & 14.786 \\
\hline & 0.752 & 0.626 & 16.645 & 1.405 & 15.240 \\
\hline & 0.754 & 0.619 & 16.915 & 1.398 & 15.517 \\
\hline & 0.739 & 0.609 & 17.215 & 1.382 & 15.833 \\
\hline
\end{tabular}

188 for the colour interval $2.15 \leq\left(V-K_{\mathrm{s}}\right)_{0} \leq 2.44$, where the metallicity of NGC $188([\mathrm{Fe} / \mathrm{H}]=-0.01 \mathrm{dex})$ fall off the metallicity interval corresponding to the cited colour interval, $-2.15 \leq[\mathrm{Fe} / \mathrm{H}] \leq-0.04$ dex, are slightly larger than those for $2.45 \leq\left(V-K_{\mathrm{s}}\right)_{0} \leq 2.60$ where the metallicity interval, $-2.15 \leq[\mathrm{Fe} / \mathrm{H}] \leq+0.37 \mathrm{dex}$, covers the metallicity of NGC 188. Although the larger residuals are given in Table 19, they are not considered in the statistics.

\section{SUMMARY AND DISCUSSION}

We calibrated the absolute magnitudes $M_{J}$ and $M_{K_{\mathrm{s}}}$ for red giants in terms of metallicity by means of the colourmagnitude diagrams of the clusters M92, M13, M71, M67, and NGC 6791 with different metallicities. The $J \times(V-J)$ and $K_{\mathrm{s}} \times\left(V-K_{\mathrm{s}}\right)$ sequences used for the calibration of $M_{J}$ and $M_{K_{\mathrm{s}}}$ are provided from different sources and by different procedures, as explained in the following. The main source is the paper of Brasseur et al. (2010). The 
Table 16. $\left(M_{J}\right)_{\mathrm{ev}}$ Absolute Magnitudes and $\Delta M_{J}$ Residuals Estimated by the Procedure Explained in Our Work. $\left(M_{J}\right)_{\mathrm{cl}}$ Denotes the Absolute Magnitude Evaluated by Means of the Colour-Magnitude Diagram of the Cluster

\begin{tabular}{|c|c|c|c|c|c|c|c|}
\hline$(V-J)_{0}$ & $\left(M_{J}\right)_{\mathrm{cl}}$ & $\left(M_{J}\right)_{\mathrm{ev}}$ & $\Delta M_{J}$ & $(V-J)_{0}$ & $\left(M_{J}\right)_{\mathrm{cl}}$ & $\left(M_{J}\right)_{\mathrm{ev}}$ & $\Delta M_{J}$ \\
\hline \multicolumn{4}{|c|}{ M5 $([\mathrm{Fe} / \mathrm{H}]=-1.17 \mathrm{dex})$} & \multicolumn{4}{|c|}{ M5 (cont.) } \\
\hline 1.40 & 1.203 & 0.876 & 0.327 & 2.38 & -4.385 & -4.417 & 0.033 \\
\hline 1.42 & 0.928 & 0.630 & 0.298 & 2.40 & -4.432 & -4.475 & 0.043 \\
\hline 1.44 & 0.670 & 0.410 & 0.260 & 2.42 & -4.474 & -4.532 & 0.058 \\
\hline 1.46 & 0.428 & 0.198 & 0.230 & 2.44 & -4.511 & -4.588 & 0.077 \\
\hline 1.48 & 0.200 & -0.005 & 0.205 & 2.46 & -4.540 & -4.642 & 0.102 \\
\hline 1.50 & -0.013 & -0.200 & 0.187 & 2.48 & -4.562 & -4.695 & 0.133 \\
\hline 1.52 & -0.214 & -0.388 & 0.173 & 2.50 & -4.577 & -4.747 & 0.170 \\
\hline 1.54 & -0.403 & -0.568 & 0.164 & \multicolumn{4}{|c|}{$\operatorname{M} 68([\mathrm{Fe} / \mathrm{H}]=-2.01 \mathrm{dex})$} \\
\hline 1.56 & -0.581 & -0.741 & 0.159 & 1.40 & 0.437 & 0.095 & 0.342 \\
\hline 1.58 & -0.749 & -0.947 & 0.198 & 1.42 & 0.140 & -0.146 & 0.286 \\
\hline 1.60 & -0.907 & -1.099 & 0.192 & 1.44 & -0.136 & -0.373 & 0.236 \\
\hline 1.62 & -1.056 & -1.245 & 0.188 & 1.46 & -0.394 & -0.588 & 0.195 \\
\hline 1.64 & -1.198 & -1.385 & 0.188 & 1.48 & -0.633 & -0.792 & 0.159 \\
\hline 1.66 & -1.332 & -1.520 & 0.189 & 1.50 & -0.855 & -0.986 & 0.131 \\
\hline 1.68 & -1.459 & -1.650 & 0.191 & 1.52 & -1.063 & -1.170 & 0.108 \\
\hline 1.70 & -1.580 & -1.775 & 0.195 & 1.54 & -1.256 & -1.346 & 0.090 \\
\hline 1.72 & -1.696 & -1.920 & 0.224 & 1.56 & -1.436 & -1.513 & 0.077 \\
\hline 1.74 & -1.807 & -2.027 & 0.220 & 1.58 & -1.604 & -1.690 & 0.086 \\
\hline 1.76 & -1.913 & -2.131 & 0.217 & 1.60 & -1.761 & -1.839 & 0.078 \\
\hline 1.78 & -2.016 & -2.231 & 0.215 & 1.62 & -1.909 & -1.982 & 0.073 \\
\hline 1.80 & -2.115 & -2.329 & 0.214 & 1.64 & -2.047 & -2.119 & 0.071 \\
\hline 1.82 & -2.211 & -2.423 & 0.212 & 1.66 & -2.178 & -2.250 & 0.072 \\
\hline 1.84 & -2.304 & -2.514 & 0.210 & 1.68 & -2.301 & -2.376 & 0.075 \\
\hline 1.86 & -2.396 & -2.603 & 0.207 & 1.70 & -2.417 & -2.498 & 0.081 \\
\hline 1.88 & -2.485 & -2.689 & 0.204 & 1.72 & -2.529 & -2.614 & 0.086 \\
\hline 1.90 & -2.573 & -2.773 & 0.200 & 1.74 & -2.635 & -2.728 & 0.094 \\
\hline 1.92 & -2.659 & -2.854 & 0.195 & 1.76 & -2.736 & -2.839 & 0.103 \\
\hline 1.94 & -2.744 & -2.933 & 0.189 & 1.78 & -2.835 & -2.946 & 0.112 \\
\hline 1.96 & -2.828 & -3.010 & 0.181 & 1.80 & -2.929 & -3.051 & 0.121 \\
\hline 1.98 & -2.912 & -3.085 & 0.173 & 1.82 & -3.022 & -3.153 & 0.131 \\
\hline 2.00 & -2.995 & -3.158 & 0.163 & 1.84 & -3.112 & -3.251 & 0.140 \\
\hline 2.02 & -3.077 & -3.229 & 0.152 & 1.86 & -3.200 & -3.348 & 0.148 \\
\hline 2.04 & -3.159 & -3.298 & 0.139 & 1.88 & -3.287 & -3.442 & 0.156 \\
\hline 2.06 & -3.240 & -3.365 & 0.125 & 1.90 & -3.373 & -3.535 & 0.162 \\
\hline 2.08 & -3.321 & -3.431 & 0.110 & 1.92 & -3.458 & -3.625 & 0.167 \\
\hline 2.10 & -3.402 & -3.496 & 0.094 & 1.94 & -3.542 & -3.714 & 0.172 \\
\hline 2.12 & -3.482 & -3.559 & 0.077 & 1.96 & -3.626 & -3.800 & 0.174 \\
\hline 2.14 & -3.561 & -3.620 & 0.059 & 1.98 & -3.709 & -3.885 & 0.176 \\
\hline 2.16 & -3.640 & -3.680 & 0.040 & 2.00 & -3.792 & -3.968 & 0.176 \\
\hline 2.18 & -3.717 & -3.739 & 0.021 & 2.02 & -3.875 & -4.049 & 0.174 \\
\hline 2.20 & -3.794 & -3.796 & 0.002 & 2.04 & -3.957 & -4.127 & 0.170 \\
\hline 2.22 & -3.869 & -3.887 & 0.018 & 2.06 & -4.039 & -4.205 & 0.165 \\
\hline 2.24 & -3.943 & -3.907 & -0.036 & 2.08 & -4.121 & -4.280 & 0.159 \\
\hline 2.26 & -4.014 & -3.960 & -0.054 & 2.10 & -4.202 & -4.352 & 0.150 \\
\hline 2.28 & -4.084 & -4.012 & -0.072 & 2.12 & -4.281 & -4.422 & 0.141 \\
\hline 2.30 & -4.151 & -4.075 & -0.076 & 2.14 & -4.360 & -4.489 & 0.129 \\
\hline 2.32 & -4.215 & -4.237 & 0.022 & 2.16 & -4.437 & -4.554 & 0.117 \\
\hline 2.34 & -4.275 & -4.298 & 0.023 & 2.18 & -4.512 & -4.616 & 0.103 \\
\hline 2.36 & -4.332 & -4.358 & 0.026 & 2.20 & -4.585 & -4.673 & 0.088 \\
\hline
\end{tabular}

$J \times(V-J)$ and $K_{\mathrm{s}} \times\left(V-K_{\mathrm{s}}\right)$ sequences for the clusters M92, M13, and M71 are taken from the tables of Brasseur et al. (2010), whereas the $J_{0} \times(V-J)_{0}$ sequence for M67 and NGC 6791 is obtained by transformation of $V, B-V$, and $V-I$ data from Montgomery et al. (1993) and by means of the $M_{V} \times(V-J)_{0}$ diagram from Brasseur et al. (2010), respectively. Also, the $K_{\mathrm{s}} \times\left(V-K_{\mathrm{s}}\right)$ sequence for M67 is transformed from $V, B-V$, and $V-I$ data in Montgomery et al. (1993). The fiducial sequence for NGC 6791 is given in $K_{\mathrm{s}} \times\left(J-K_{\mathrm{s}}\right)$ in Brasseur et al. (2010). We 
Table 17. Distribution of the Residuals. $N$ Denotes the Number of Stars

\begin{tabular}{lrr}
\hline \hline$\Delta M_{J}$ Interval & $\left\langle\Delta M_{J}\right\rangle$ & $N$ \\
\hline$(-0.1,0.0]$ & -0.059 & 4 \\
$(0.0,0.1]$ & 0.060 & 26 \\
$(0.1,0.2]$ & 0.158 & 50 \\
$(0.2,0.3]$ & 0.229 & 15 \\
$(0.3,0.4]$ & 0.335 & 2 \\
\hline \hline
\end{tabular}

Table 18. $K_{\mathrm{s}} \times\left(V-K_{\mathrm{s}}\right)_{0}$ Fiducial Giant Sequences for the Clusters NGC 188 and M68 Used in the Application of the Procedure

\begin{tabular}{lccccc}
\hline \hline Cluster & $V_{0}$ & $(B-V)_{0}$ & $(V-I)_{0}$ & $\left(V-K_{\mathrm{s}}\right)_{0}$ & $K_{\mathrm{s}_{0}}$ \\
\hline NGC 188 & 12.481 & 1.119 & 1.112 & 2.608 & 9.873 \\
& 13.031 & 1.052 & 1.063 & 2.474 & 10.557 \\
& 13.547 & 0.999 & 1.022 & 2.367 & 11.180 \\
& 14.069 & 0.954 & 0.991 & 2.277 & 11.792 \\
& 14.609 & 0.839 & 0.899 & 2.044 & 12.565 \\
& 14.786 & 0.819 & 0.885 & 2.004 & 12.782 \\
M68 & 12.497 & 1.196 & 1.234 & 3.040 & 9.457 \\
& 12.844 & 1.154 & 1.202 & 2.955 & 9.889 \\
& 13.498 & 0.951 & 1.047 & 2.546 & 10.952 \\
& 14.143 & 0.855 & 0.965 & 2.350 & 11.793 \\
& 14.744 & 0.775 & 0.905 & 2.189 & 12.555 \\
& 15.209 & 0.732 & 0.863 & 2.100 & 13.109 \\
& 15.775 & 0.669 & 0.803 & 1.970 & 13.805 \\
& 16.228 & 0.646 & 0.792 & 1.925 & 14.303 \\
& 16.645 & 0.626 & 0.752 & 1.878 & 14.767 \\
& 16.915 & 0.619 & 0.754 & 1.866 & 15.049 \\
& 17.215 & 0.609 & 0.739 & 1.843 & 15.372 \\
\hline \hline
\end{tabular}

transformed the $J_{0}$ magnitudes to the $V_{0}$ ones obtained from the $M_{V} \times(V-J)_{0}$ diagram and altered the $K_{\mathrm{s}} \times\left(J-K_{\mathrm{s}}\right)$ data to $K_{\mathrm{s}} \times\left(V-K_{\mathrm{s}}\right)$ ones. Thus, we obtained two sets of data for two absolute magnitude calibrations, i.e. $J_{0} \times(V-$ $J)_{0}$ and $K_{\mathrm{s}_{0}} \times\left(V-K_{\mathrm{s}}\right)_{0}$ for $M_{J}$ and $M_{K_{\mathrm{s}}}$, respectively. We combined each set of data for each cluster with their true distance modulus and evaluated two sets of absolute magnitudes for the $(V-J)_{0}$ and $\left(V-K_{\mathrm{s}}\right)_{0}$ ranges of each cluster. Then, we fitted $M_{J}$ and $M_{K_{s}}$ absolute magnitudes in terms of iron metallicity, $[\mathrm{Fe} / \mathrm{H}]$, by quadratic polynomials, for a given $(V-J)_{0}$ and $\left(V-K_{\mathrm{s}}\right)_{0}$ colour index, respectively. The calibrations cover large ranges, i.e. $1.30 \leq(V-J)_{0} \leq 2.80$ and $1.75 \leq\left(V-K_{\mathrm{s}}\right)_{0} \leq 3.80 \mathrm{mag}$ for $M_{J}$ and $M_{K_{\mathrm{s}}}$, respectively.

We evaluated the $M_{J}$ absolute magnitudes of the clusters M5 $([\mathrm{Fe} / \mathrm{H}]=-1.17 \mathrm{dex})$ and M68 $([\mathrm{Fe} / \mathrm{H}]=-2.01$ dex) by the procedure presented in our study for a set of $(V-J)_{0}$ colour index and compared them with those estimated via combination of the fiducial $J_{0} \times(V-J)_{0}$ sequence and the true distance modulus for each cluster. The total of the residuals lie between -0.08 and $+0.34 \mathrm{mag}$, and the range of $94 \%$ of them is $0<\Delta M_{J} \leq 0.3 \mathrm{mag}$. The mean and the standard deviation of (all) the residuals are $\left\langle\Delta M_{J}\right\rangle=0.137$ and $\sigma_{M_{I}}=0.080 \mathrm{mag}$. For the evaluation of the $M_{K_{\mathrm{s}}}$ absolute magnitudes, we applied the corresponding procedure to the clusters NGC $188([\mathrm{Fe} / \mathrm{H}]=-0.01 \mathrm{dex})$ and M68 $([\mathrm{Fe} / \mathrm{H}]=-2.01 \mathrm{dex})$. Here again, the range of the residuals, their mean and standard deviation are small, i.e. $-0.10<\Delta M_{K_{\mathrm{s}}} \leq+0.27 \mathrm{mag},\left\langle\Delta M_{K_{\mathrm{s}}}\right\rangle=0.109$, and $\sigma_{M_{\mathrm{s}}}=0.123 \mathrm{mag}$.

We compared the statistical results obtained in this study with those of Papers I and II. Table 21 shows that $M_{V}, M_{g}, M_{J}$, and $M_{K_{\mathrm{s}}}$ absolute magnitudes can be estimated with an error less than $0.3 \mathrm{mag}$. However, one can notice an improvement on $M_{J}$ and $M_{K_{\mathrm{s}}}$ with respect to $M_{V}$ and $M_{g}$. The main difference between the data of the clusters in three studies is the large domain of the clusters in $(V-J)_{0}$ and $\left(V-K_{\mathrm{s}}\right)_{0}$ which probably contributed to more accurate calibrations of the apparent $J_{0}$ and $K_{\mathrm{s}}$ magnitudes in terms of the corresponding colours with respect to $(B-V)_{0}$ and $(g-r)_{0}$ ones. Accurate calibration in apparent magnitude provided accurate absolute magnitudes. The magnitudes and colours for the cluster M67 used in the calibration of $M_{J}$ and $M_{K}$ absolute magnitudes are not original, but they are transformed from the $V, B-V$, and $V-I$ data by means of the equations of Yaz et al. (2010). The same case holds for the clusters NGC 188 and M68 which are used in the application of the procedure. Calibrations with high correlation coefficients and small residuals also confirm the equations of Yaz et al. (2010). As claimed in Papers I and II, there was an improvement in the results therein with respect to those of Hog \& Flynn (1998). Hence, the same improvement holds for this study. We also quote the work of Ljunggren \& Oja (1966).

Although age plays an important role in the trend of the fiducial sequence of the RGB, we have not used it as a parameter in the calibration of absolute magnitude. A quadratic calibration in terms of (only) metallicity provides absolute magnitudes with high accuracy. Another problem may originate from the red clump (RC) stars. These stars lie very close to the RGB but they present a completely different group of stars. Tables 16 and 19 and Figures 7 and 8 summarise how reliable are our absolute magnitudes. If age and possibly the mix with RC stars would affect our results, this should up. In addition, we should add that the fiducial sequences used in our study were properly selected as RGB. However, the researchers should identify and exclude the RC stars when they apply our calibrations to the field stars.

The accuracy of the estimated absolute magnitudes depends mainly on the accuracy of metallicity. We altered the metallicity by $[\mathrm{Fe} / \mathrm{H}]+\Delta[\mathrm{Fe} / \mathrm{H}]$ in evaluation of the absolute magnitudes by the procedure presented in our study and we checked its effect on the absolute magnitude. We adopted $[\mathrm{Fe} / \mathrm{H}]=-2.01,-1.117,-0.01$ dex and $\Delta[\mathrm{Fe} / \mathrm{H}]=0.05,0.10,0.15,0.20 \mathrm{dex}$ and re-evaluated the absolute magnitudes for ten $(V-J)_{0}$ and nine $\left(V-K_{\mathrm{s}}\right)$ colour indices for this purpose. The differences between the absolute magnitudes evaluated in this way and the corresponding ones evaluated without $\Delta[\mathrm{Fe} / \mathrm{H}]$ increments are given in Table 22. The maximum absolute magnitude differences corresponding to $\Delta[\mathrm{Fe} / \mathrm{H}]=0.20 \mathrm{dex}$ in $M_{J}$ and $M_{K_{\mathrm{s}}}$ 
Table 19. $\left(M_{K_{\mathrm{s}}}\right)_{\mathrm{ev}}$ Absolute Magnitudes and $\Delta M_{K_{\mathrm{s}}}$ Residuals Estimated by the Procedure Explained in Our Work. $\left(M_{K_{\mathrm{s}}}\right)_{\mathrm{cl}}$ Denotes the Absolute Magnitude Evaluated by Means of the Colour-Magnitude Diagram of the Cluster

\begin{tabular}{|c|c|c|c|c|c|c|c|c|c|c|c|}
\hline$\left(V-K_{\mathrm{s}}\right)_{0}$ & $\left(M_{K_{\mathrm{s}}}\right)_{\mathrm{cl}}$ & $\left(M_{K_{\mathrm{s}}}\right)_{\mathrm{ev}}$ & $\Delta M_{K_{\mathrm{s}}}$ & $\left(V-K_{\mathrm{s}}\right)_{0}$ & $\left(M_{K_{\mathrm{s}}}\right)_{\mathrm{cl}}$ & $\left(M_{K_{\mathrm{s}}}\right)_{\mathrm{ev}}$ & $\Delta M_{K_{\mathrm{s}}}$ & $\left(V-K_{\mathrm{s}}\right)_{0}$ & $\left(M_{K_{\mathrm{s}}}\right)_{\mathrm{cl}}$ & $\left(M_{K_{\mathrm{s}}}\right)_{\mathrm{ev}}$ & $\Delta M_{K_{\mathrm{s}}}$ \\
\hline \multicolumn{4}{|c|}{$\mathrm{NGC} 188([\mathrm{Fe} / \mathrm{H}]=-0.01 \mathrm{dex})$} & \multicolumn{4}{|c|}{ M68 (cont.) } & \multicolumn{4}{|c|}{ M68 (cont.) } \\
\hline 2.15 & 1.092 & 1.029 & 0.063 & 1.93 & -0.671 & -0.691 & 0.020 & 2.49 & -3.735 & -3.876 & 0.141 \\
\hline 2.16 & 1.061 & 0.963 & 0.097 & 1.94 & -0.765 & -0.771 & 0.006 & 2.50 & -3.768 & -3.917 & 0.149 \\
\hline 2.17 & 1.029 & 0.898 & 0.131 & 1.95 & -0.857 & -0.850 & -0.007 & 2.51 & -3.802 & -3.958 & 0.156 \\
\hline 2.18 & 0.996 & 0.832 & 0.163 & 1.96 & -0.947 & -0.928 & -0.020 & 2.52 & -3.835 & -3.999 & 0.164 \\
\hline 2.19 & 0.962 & 0.767 & 0.194 & 1.97 & -1.035 & -1.004 & -0.031 & 2.53 & -3.868 & -4.039 & 0.171 \\
\hline 2.20 & 0.926 & 0.702 & 0.224 & 1.98 & -1.121 & -1.079 & -0.041 & 2.54 & -3.902 & -4.079 & 0.178 \\
\hline 2.21 & 0.890 & 0.638 & 0.252 & 1.99 & -1.204 & -1.155 & -0.050 & 2.55 & -3.934 & -4.120 & 0.185 \\
\hline 2.22 & 0.852 & 0.574 & 0.278 & 2.00 & -1.286 & -1.227 & -0.059 & 2.56 & -3.967 & -4.159 & 0.191 \\
\hline 2.23 & 0.812 & 0.511 & 0.302 & 2.01 & -1.365 & -1.298 & -0.067 & 2.57 & -4.000 & -4.197 & 0.198 \\
\hline 2.24 & 0.771 & 0.447 & 0.324 & 2.02 & -1.443 & -1.369 & -0.074 & 2.58 & -4.032 & -4.236 & 0.204 \\
\hline 2.25 & 0.728 & 0.385 & 0.344 & 2.03 & -1.518 & -1.439 & -0.080 & 2.59 & -4.065 & -4.275 & 0.210 \\
\hline 2.26 & 0.684 & 0.322 & 0.361 & 2.04 & -1.592 & -1.508 & -0.085 & 2.60 & -4.097 & -4.313 & 0.216 \\
\hline 2.27 & 0.637 & 0.261 & 0.377 & 2.05 & -1.664 & -1.575 & -0.089 & 2.61 & -4.129 & -4.351 & 0.221 \\
\hline 2.28 & 0.589 & 0.199 & 0.390 & 2.06 & -1.735 & -1.642 & -0.093 & 2.62 & -4.162 & -4.388 & 0.227 \\
\hline 2.29 & 0.539 & 0.139 & 0.401 & 2.07 & -1.803 & -1.707 & -0.096 & 2.63 & -4.194 & -4.426 & 0.232 \\
\hline 2.30 & 0.488 & 0.078 & 0.409 & 2.08 & -1.870 & -1.772 & -0.098 & 2.64 & -4.226 & -4.463 & 0.237 \\
\hline 2.31 & 0.434 & 0.019 & 0.415 & 2.09 & -1.936 & -1.836 & -0.100 & 2.65 & -4.258 & -4.499 & 0.241 \\
\hline 2.32 & 0.379 & -0.143 & 0.522 & 2.10 & -2.000 & -1.899 & -0.101 & 2.66 & -4.290 & -4.535 & 0.246 \\
\hline 2.33 & 0.322 & -0.099 & 0.421 & 2.11 & -2.062 & -1.961 & -0.102 & 2.67 & -4.322 & -4.571 & 0.250 \\
\hline 2.34 & 0.264 & -0.157 & 0.421 & 2.12 & -2.124 & -2.022 & -0.101 & 2.68 & -4.354 & -4.607 & 0.253 \\
\hline 2.35 & 0.204 & -0.215 & 0.418 & 2.13 & -2.183 & -2.082 & -0.101 & 2.69 & -4.386 & -4.642 & 0.256 \\
\hline 2.36 & 0.142 & -0.271 & 0.414 & 2.14 & -2.242 & -2.142 & -0.099 & 2.70 & -4.418 & -4.677 & 0.259 \\
\hline 2.37 & 0.080 & -0.328 & 0.407 & 2.15 & -2.299 & -2.223 & -0.076 & 2.71 & -4.449 & -4.712 & 0.262 \\
\hline 2.38 & 0.016 & -0.383 & 0.399 & 2.16 & -2.355 & -2.281 & -0.074 & 2.72 & -4.481 & -4.746 & 0.265 \\
\hline 2.39 & -0.049 & -0.439 & 0.389 & 2.17 & -2.409 & -2.338 & -0.071 & 2.73 & -4.513 & -4.780 & 0.267 \\
\hline 2.40 & -0.115 & -0.493 & 0.378 & 2.18 & -2.463 & -2.395 & -0.068 & 2.74 & -4.545 & -4.814 & 0.269 \\
\hline 2.41 & -0.181 & -0.547 & 0.366 & 2.19 & -2.515 & -2.451 & -0.064 & 2.75 & -4.577 & -4.847 & 0.270 \\
\hline 2.42 & -0.248 & -0.600 & 0.352 & 2.20 & -2.566 & -2.506 & -0.060 & 2.76 & -4.608 & -4.880 & 0.271 \\
\hline 2.43 & -0.316 & -0.653 & 0.337 & 2.21 & -2.616 & -2.560 & -0.056 & 2.77 & -4.640 & -4.912 & 0.272 \\
\hline 2.44 & -0.383 & -0.705 & 0.322 & 2.22 & -2.665 & -2.614 & -0.051 & 2.78 & -4.672 & -4.944 & 0.272 \\
\hline 2.45 & -0.450 & -0.634 & 0.184 & 2.23 & -2.714 & -2.668 & -0.046 & 2.79 & -4.704 & -4.976 & 0.272 \\
\hline 2.46 & -0.517 & -0.691 & 0.174 & 2.24 & -2.761 & -2.720 & -0.041 & 2.80 & -4.735 & -5.007 & 0.272 \\
\hline 2.47 & -0.583 & -0.747 & 0.163 & 2.25 & -2.807 & -2.772 & -0.035 & 2.81 & -4.767 & -5.038 & 0.271 \\
\hline 2.48 & -0.649 & -0.801 & 0.152 & 2.26 & -2.853 & -2.824 & -0.029 & 2.82 & -4.798 & -5.068 & 0.270 \\
\hline 2.49 & -0.713 & -0.855 & 0.142 & 2.27 & -2.898 & -2.875 & -0.023 & 2.83 & -4.830 & -5.098 & 0.268 \\
\hline 2.50 & -0.775 & -0.908 & 0.133 & 2.28 & -2.941 & -2.925 & -0.016 & 2.84 & -4.861 & -5.127 & 0.266 \\
\hline 2.51 & -0.836 & -0.960 & 0.124 & 2.29 & -2.985 & -2.975 & -0.010 & 2.85 & -4.893 & -5.156 & 0.264 \\
\hline 2.52 & -0.894 & -1.011 & 0.117 & 2.30 & -3.027 & -3.024 & -0.003 & 2.86 & -4.924 & -5.185 & 0.261 \\
\hline 2.53 & -0.950 & -1.061 & 0.111 & 2.31 & -3.069 & -3.073 & 0.004 & 2.87 & -4.955 & -5.213 & 0.258 \\
\hline 2.54 & -1.003 & -1.111 & 0.107 & 2.32 & -3.110 & -3.224 & 0.114 & 2.88 & -4.986 & -5.241 & 0.255 \\
\hline 2.55 & -1.053 & -1.159 & 0.106 & 2.33 & -3.150 & -3.169 & 0.019 & 2.89 & -5.017 & -5.268 & 0.251 \\
\hline 2.56 & -1.099 & -1.207 & 0.108 & 2.34 & -3.190 & -3.217 & 0.026 & 2.90 & -5.048 & -5.294 & 0.247 \\
\hline 2.57 & -1.141 & -1.254 & 0.113 & 2.35 & -3.230 & -3.264 & 0.034 & 2.91 & -5.078 & -5.321 & 0.242 \\
\hline 2.58 & -1.178 & -1.300 & 0.122 & 2.36 & -3.268 & -3.310 & 0.042 & 2.92 & -5.109 & -5.346 & 0.237 \\
\hline 2.59 & -1.210 & -1.345 & 0.135 & 2.37 & -3.307 & -3.356 & 0.050 & 2.93 & -5.139 & -5.370 & 0.231 \\
\hline 2.60 & -1.237 & -1.390 & 0.153 & 2.38 & -3.344 & -3.402 & 0.057 & 2.94 & -5.169 & -5.395 & 0.225 \\
\hline \multicolumn{4}{|c|}{$\operatorname{M68}([\mathrm{Fe} / \mathrm{H}]=-2.01 \mathrm{dex})$} & 2.39 & -3.382 & -3.447 & 0.065 & 2.95 & -5.199 & -5.418 & 0.219 \\
\hline 1.84 & 0.295 & 0.096 & 0.199 & 2.40 & -3.419 & -3.492 & 0.073 & 2.96 & -5.229 & -5.441 & 0.212 \\
\hline 1.85 & 0.177 & 0.002 & 0.175 & 2.41 & -3.455 & -3.536 & 0.081 & 2.97 & -5.259 & -5.464 & 0.205 \\
\hline 1.86 & 0.061 & -0.090 & 0.151 & 2.42 & -3.491 & -3.580 & 0.089 & 2.98 & -5.288 & -5.486 & 0.198 \\
\hline 1.87 & -0.051 & -0.180 & 0.129 & 2.43 & -3.527 & -3.624 & 0.097 & 2.99 & -5.317 & -5.507 & 0.190 \\
\hline 1.88 & -0.161 & -0.269 & 0.108 & 2.44 & -3.562 & -3.667 & 0.105 & 3.00 & -5.346 & -5.527 & 0.181 \\
\hline 1.89 & -0.268 & -0.356 & 0.088 & 2.45 & -3.597 & -3.707 & 0.110 & 3.01 & -5.374 & -5.547 & 0.173 \\
\hline 1.90 & -0.372 & -0.442 & 0.069 & 2.46 & -3.632 & -3.750 & 0.118 & 3.02 & -5.402 & -5.566 & 0.164 \\
\hline 1.91 & -0.474 & -0.526 & 0.052 & 2.47 & -3.666 & -3.792 & 0.126 & 3.03 & -5.430 & -5.583 & 0.154 \\
\hline 1.92 & -0.574 & -0.609 & 0.035 & 2.48 & -3.701 & -3.834 & 0.133 & 3.04 & -5.457 & -5.601 & 0.144 \\
\hline
\end{tabular}


Table 20. Distribution of the Residuals. $N$ Denotes the Number of Stars

\begin{tabular}{lrr}
\hline \hline$\Delta M_{K_{\mathrm{s}}}$ Interval & $\left\langle\Delta M_{K_{\mathrm{s}}}\right\rangle$ & $\mathrm{N}$ \\
\hline$(-0.2,-0.1]$ & -0.101 & 5 \\
$(-0.1,0.0]$ & -0.055 & 31 \\
$(0.0,0.1]$ & 0.050 & 18 \\
$(0.1,0.2]$ & 0.148 & 43 \\
$(0.2,0.3]$ & 0.248 & 40 \\
\hline \hline
\end{tabular}

lie in the intervals $0.09 \leq \Delta M_{J} \leq 0.31$ and $0.05 \leq \Delta M_{K_{\mathrm{s}}} \leq$ 0.14 , respectively, for the metallicities $[\mathrm{Fe} / \mathrm{H}]=-1.117$ and $[\mathrm{Fe} / \mathrm{H}]=-2.01 \mathrm{dex}$. However, they are about $0.5 \mathrm{mag}$ for the metallicity $[\mathrm{Fe} / \mathrm{H}]=-0.01 \mathrm{dex}$. The mean error in metallicity for 42 globular and 33 open clusters in the catalogue of Santos \& Piatti (2004) is $\sigma=0.19$ dex. If we assume the same error for the field stars, the probable error in $M_{J}$ and $M_{K_{\mathrm{s}}}$ would be less than $0.3 \mathrm{mag}$ for relatively metal-poor stars. However, for the solar metallicity stars, the metallicity error should be $\sigma_{[\mathrm{Fe} / \mathrm{H}]}<0.15$ dex in order to estimate accurate absolute magnitudes. That is, the solar metallicities should be determined more preciously.

The absolute magnitudes can be calibrated as a function of ultraviolet excess instead of metallicity, in general. However, an ultraviolet band is not defined in 2MASS photometry. Hence, we calibrated the $M_{J}$ and $M_{K_{\mathrm{s}}}$ absolute magnitudes
Table 21. Comparison of the Results in Three Studies. The Word "all" Indicates all the Residuals. A Subset of the Residuals Is Denoted by a Percentage, Such as $91 \%$ or $94 \%$

\begin{tabular}{lccc}
\hline \hline$\Delta M$ Range & $\langle\Delta M\rangle$ & $\sigma$ & Study \\
\hline$(-0.61,+0.66)($ all $)$ & $0.05(91 \%)$ & $0.190(91 \%)$ & Paper I \\
{$[-0.40,0.40](91 \%)$} & & & \\
$(-0.28,+0.43]($ all $)$ & $0.169($ all $)$ & $0.140($ all $)$ & Paper II \\
$(0.10,0.40],(94 \%)$ & & & \\
$(-0.08,+0.34]($ all $)$ & 0.137 (all) & $0.080($ all $)$ & This study, $\Delta M_{J}$ \\
$(0.00,0.30] 94 \%$ & 0.109 & 0.123 & This study, $\Delta M_{K_{\mathrm{s}}}$ \\
$(-0.10,+0.27]$ & & & \\
\hline \hline
\end{tabular}

in terms of metallicity which can be determined by means of atmospheric model parameters. Age is a secondary parameter for the old clusters and does not influence much the position of their RGB. The youngest cluster in our study is M67 with an age of 4 Gyr (Paper I). However, the field stars may be younger. Recall that the derived relations are applicable to stars older than 4 Gyr.

We conclude that the two absolute magnitudes, $M_{J}$ and $M_{K}$, in 2MASS photometry can be estimated for the red giants in terms of metallicity with an accuracy of $\Delta M \leq 0.3$ mag. Our target in the near future would be to adopt this procedure to RC stars.

Table 22. Absolute Magnitudes Estimated by Altering the Metallicity as $[\mathrm{Fe} / \mathrm{H}]+\Delta[\mathrm{Fe} / \mathrm{H}]$

\begin{tabular}{|c|c|c|c|c|c|c|c|c|c|c|}
\hline \multirow[b]{2}{*}{$(V-J)_{0}$} & \multicolumn{5}{|c|}{$M_{J}$} & \multicolumn{4}{|c|}{$\Delta M_{J}$} & \\
\hline & (1) & (2) & (3) & (4) & (5) & (6) & (7) & (8) & (9) & \\
\hline 1.50 & -0.200 & -0.139 & -0.076 & -0.012 & 0.055 & 0.061 & 0.124 & 0.189 & 0.255 & \\
\hline 1.70 & -1.775 & -1.703 & -1.629 & -1.550 & -1.469 & 0.072 & 0.147 & 0.225 & 0.306 & \\
\hline 1.90 & -2.773 & -2.707 & -2.639 & -2.568 & -2.496 & 0.066 & 0.134 & 0.204 & 0.277 & {$[\mathrm{Fe} / \mathrm{H}]=-1.117+\Delta[\mathrm{Fe} / \mathrm{H}$} \\
\hline 2.10 & -3.496 & -3.431 & -3.365 & -3.298 & -3.229 & 0.064 & 0.130 & 0.198 & 0.267 & \\
\hline 2.30 & -4.075 & -4.007 & -3.938 & -3.868 & -3.798 & 0.068 & 0.137 & 0.207 & 0.277 & \\
\hline 2.50 & -4.747 & -4.707 & -4.664 & -4.619 & -4.570 & 0.040 & 0.082 & 0.128 & 0.177 & \\
\hline 1.50 & -0.986 & -0.952 & -0.917 & -0.879 & -0.841 & 0.034 & 0.070 & 0.107 & 0.146 & \\
\hline 1.70 & -2.498 & -2.481 & -2.460 & -2.436 & -2.408 & 0.018 & 0.038 & 0.062 & 0.090 & {$[\mathrm{Fe} / \mathrm{H}]=-2.01+\Delta[\mathrm{Fe} / \mathrm{H}]$} \\
\hline 1.90 & -3.535 & -3.508 & -3.478 & -3.446 & -3.412 & 0.027 & 0.057 & 0.088 & 0.123 & \\
\hline \multirow[t]{2}{*}{2.10} & -4.352 & -4.313 & -4.272 & -4.230 & -4.187 & 0.039 & 0.079 & 0.121 & 0.165 & \\
\hline & \multicolumn{5}{|c|}{$M_{K_{\mathrm{s}}}$} & \multicolumn{4}{|c|}{$\Delta M_{K_{\mathrm{s}}}$} & \\
\hline$\left(V-K_{\mathrm{s}}\right)_{0}$ & (1) & (2) & (3) & (4) & (5) & (6) & (7) & (8) & (9) & \\
\hline 2.45 & -0.634 & -0.489 & -0.341 & -0.190 & -0.035 & 0.145 & 0.293 & 0.445 & 0.599 & \\
\hline 2.50 & -0.908 & -0.772 & -0.633 & -0.491 & -0.346 & 0.136 & 0.275 & 0.417 & 0.562 & {$[\mathrm{Fe} / \mathrm{H}]=-0.01+\Delta[\mathrm{Fe} / \mathrm{H}]$} \\
\hline 2.55 & -1.159 & -1.031 & -0.900 & -0.766 & -0.630 & 0.128 & 0.259 & 0.393 & 0.529 & \\
\hline 2.60 & -1.390 & -1.268 & -1.144 & -1.018 & -0.889 & 0.122 & 0.245 & 0.372 & 0.500 & \\
\hline 1.85 & 0.002 & 0.024 & 0.048 & 0.074 & 0.103 & 0.022 & 0.046 & 0.072 & 0.101 & \\
\hline 2.05 & -1.575 & -1.557 & -1.536 & -1.513 & -1.487 & 0.018 & 0.039 & 0.063 & 0.089 & \\
\hline 2.25 & -2.772 & -2.764 & -2.753 & -2.738 & -2.719 & 0.008 & 0.019 & 0.034 & 0.053 & {$[\mathrm{Fe} / \mathrm{H}]=-2.01+\Delta[\mathrm{Fe} / \mathrm{H}]$} \\
\hline 2.45 & -3.707 & -3.695 & -3.679 & -3.661 & -3.639 & 0.012 & 0.027 & 0.046 & 0.068 & \\
\hline 2.65 & -4.499 & -4.468 & -4.434 & -4.398 & -4.361 & 0.031 & 0.065 & 0.101 & 0.138 & \\
\hline
\end{tabular}

Notes. The numerical values of $[\mathrm{Fe} / \mathrm{H}]$ are indicated in the last column. The absolute magnitudes in column (1) are the original ones taken from Tables 16 and 19, whereas those in columns (2)-(5) correspond to the increments $0.05,0.10,0.15$, and 0.20 dex. The differences between the original absolute magnitudes and those evaluated by means of the increments are given in columns (6)-(9). 


\section{ACKNOWLEDGMENTS}

This research has made use of NASA's Astrophysics Data System and the SIMBAD database, operated at CDS, Strasbourg, France

\section{REFERENCES}

Anthony-Twarog, B. J., Twarog, B. A., \& Mayer, L. 2007, AJ, 133, 1585

Bilir, S., Karaali, S., Ak, S., Coşkunoğlu, K. B., Yaz, E., \& CabreraLavers, A. 2009, MNRAS, 396, 1589

Bilir, S., Karaali, S., Ak, S., Yaz, E., Cabrera-Lavers, A., \& Coşkunoğlu, K. B. 2008, MNRAS, 390, 1569

Bilir, S., Karaali, S., Dağtekin, N. D., Önal, Ö., Ak, S., Ak, T., \& Cabrera-Lavers, A. 2012, PASA, 29, 121

Brasseur, C. M., Stetson, P. B., VandenBerg, D. A., Casagrande, L., Bono, G., \& Dall'Ora, M. 2010, AJ, 140, 1672

Breddels, M. A., et al. 2010, A\&A, 511A, 90

Chen, B., et al. 2001, ApJ, 553, 184

ESA 1997, The Hipparcos and Tycho Catalogues (ESA SP-1200; Noordwijk: ESA)

Fiorucci, M., \& Munari, U. 2003, A\&A, 401, 781

Gratton, R. G. Fusi Pecci, F., Carretta, E., Clementini, G., Corsi, C. E., \& Lattanzi, M. 1997, ApJ, 491, 749

Harris, W. E. 2010, arXiv1012.3224

Hodder, P. J. C., Nemec, J. M., Richer, H. B., \& Fahlman, G. G. 1992, AJ, 103, 460

Hog, E., \& Flynn, C. 1998, MNRAS, 294, 28

Karaali, S., Bilir, S., \& Yaz Gökçe, E. 2012a, PASA, 29, 509

Karaali, S., Bilir, S., \& Yaz Gökçe, E. 2012b, PASA, in press (arXiv:1206.2752K) (Paper II)

Karaali, S., Karataş, Y., Bilir, S., Ak, S. G., \& Hamzaoğlu, E. 2003, PASA, 20, 270

Laird, J. B., Carney, B. W., \& Latham, D. W. 1988, AJ, 96, 1908
Ljunggren, B., \& Oja, T. 1966, IAUS, 24, 317

McCall, M. L. 2004, AJ, 128, 2144

Meibom, S., et al. 2009, AJ, 137, 5086

Montgomery, K. A., Marschall, L. A., \& Janes, K. A. 1993, AJ, 106,181

Nissen, P. E., \& Schuster, W. J. 1991, A\&A, 251, 457

Phleps, S., Meisenheimer, K., Fuchs, B., \& Wolf, C. 2000, A\&A, 356, 108

Rosenberg, A., Saviane, I., Piotto, G., \& Aparicio, A. 1999, AJ, 118, 2306

Sandquist, E. L., Bolte, M., Stetson, P. B., \& Hesser, J. E. 1996, ApJ, 470, 910

Santos, J. F. C. Jr, \& Piatti, A. E. 2004, A\&A, 428, 79

Sandage, A., Lubin, L.M., \& VandenBerg, D.A. 2003, PASP, 115, 1187

Sarajedini, A., Dotter, A., \& Kirkpatrick, A. 2009, ApJ, 698, 1872

Savage, B. D., \& Mathis, J. S. 1979, ARA\&A, 17, 73

Saviane, I., Piotto, G., Fagotto, F., Zaggia, S., Capaccioli, M., \& Aparicio, A. 1998, A\&A, 333, 479

Siebert A., et al. 2011, AJ, 141, 187

Siegel, M. H., Majewski, S. R., Reid, I. N., \& Thompson, I. B. 2002, ApJ, 578, 151

Skrutskie, M. F., et al. 2006, AJ, 131, 1163

Smith, R. G. 1987, MNRAS, 227, 943

Stetson, P. B., McClure, R. D., \& VandenBerg, D. 2004, PASP, 116, 1012

Turner, D. G. 2011, RMxAA, 47, 127

Turner, D. G. 2012, Ap\&SS, 337, 303

van Leeuwen, F. 2007, A\&A, 474, 653

VandenBerg, D. A., \& Clem, J. L. 2003, AJ, 126, 778

Walker, A. R. 1994, AJ, 108, 555

Yaz, E., Bilir, S., Karaali, S., Ak, S., Coşkunoğlu, B., \& CabreraLavers, A. 2010, AN, 331, 807

Zwitter, T., et al. 2010, A\&A, 522A, 54 